\title{
Comparison of several difference schemes on 1D and 2D test problems for the Euler equations
}

\author{
Richard Liska $^{1}$, Burton Wendroff ${ }^{2}$ \\ ${ }^{1}$ Faculty of Nuclear Sciences and Physical Engineering \\ Czech Technical University in Prague \\ Břehová 7, 11519 Prague 1, Czech Republic \\ liska@siduri.fjfi.cvut.cz \\ http://www-troja.fjfi.cvut.cz/ Iiska \\ ${ }^{2}$ Group T-7, Los Alamos National Laboratory \\ Los Alamos, NM 87544, USA \\ bbw@lanl.gov \\ http://math.unm.edu/ ${ }^{\sim}$ bbw
}

November 22, 2001 


\section{Contents}

\begin{tabular}{l|r}
\hline Introduction & 3
\end{tabular}

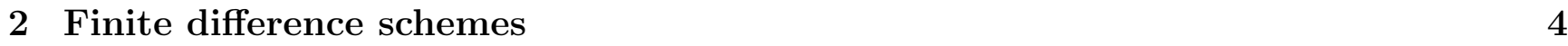

2.1 A composite scheme - $\mathrm{CFLH}$. . . . . . . . . . . . . . . . . . . . . . . 4

2.2 A CFLF hybrid scheme-CFLFh . . . . . . . . . . . . . . . . . . . 4

2.3 Centered scheme with limiter - J] . . . . . . . . . . . . . . . . . . . 5 5

2.4 Positive scheme $-\mathrm{L}$. . . . . . . . . . . . . . . . . . . . . . 5

2.5 Clawpack wave propagation scheme - CLAW . . . . . . . . . . . . . . . 5

2.6 Weighted average flux (WAF) schemes - WAFT and WAFC . . . . . . . . . . . . 5

2.7 Weighted essentially nonoscillatory schemes - WENO5 and CWENO3 . . . . . . . 5

2.8 Piecewise parabolic - PPM . . . . . . . . . . . . . . . . . . . . . 6

$\begin{array}{ll}3 \text { 1D tests } & 7\end{array}$

3.1 Description of $1 \mathrm{D}$ problems $\ldots \ldots \ldots \ldots \ldots$. . . . . . . . . . . . . . . 7

3.2 Errors of the numerical solution . . . . . . . . . . . . . . . . . . . . . . . . . . . . . . . 8

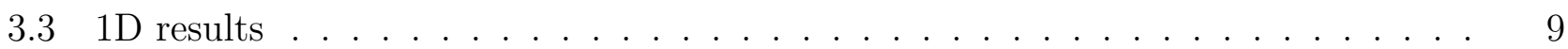

$\begin{array}{lll}42 \mathrm{D} \text { tests } & 23\end{array}$

4.1 Accuracy - Smooth periodic problem . . . . . . . . . . . . . . . . . . . . . . . 23

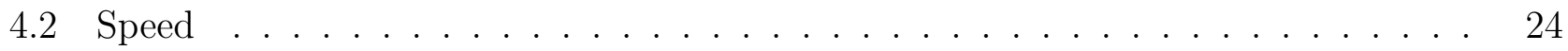

4.3 Description of $2 \mathrm{D}$ Riemann problems . . . . . . . . . . . . . . . . . . . . . . . . . . . . . . . . . . . . .

4.4 Results for 2D Riemann problems . . . . . . . . . . . . . . . . . . . . . . . . . . . 26

4.5 Noh problem . . . . . . . . . . . . . . . . . . . . . . . . . . . . . . 39

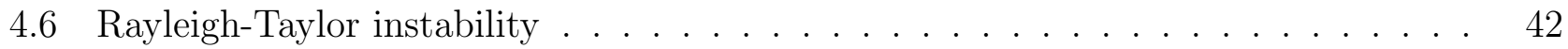

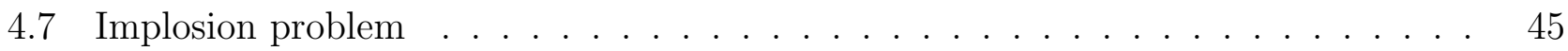

4.8 Explosion . . . . . . . . . . . . . . . . . . . . . . . . . 51

\begin{tabular}{ll|lr}
5 Final remarks & 54
\end{tabular} 


\begin{abstract}
The results of computations with ten finite difference schemes on a suite of one-dimensional and two-dimensional test problems for the Euler equations are presented in various formats.
\end{abstract}

Keywords: Euler, Riemann problems, finite difference schemes, splitting

\title{
1 Introduction
}

Hyperbolic conservation laws, and the Euler equations of compressible fluid dynamics in particular, have been the subject of intensive research for at least the past five decades, and with good reason. The applications are many - aircraft design, stellar formation, weather prediction to name only a few. There are some theoretical results [ [1, 2, 3], and we strongly recommend the pre-print server web site [4] for the many papers there on theoretical and numerical aspects of hyperbolic conservation laws. Even if the theory were perfect the applications would not be possible without methods for obtaining approximate solutions. The unfortunate situation here is that rigorous error estimates for supposed approximate solutions are almost entirely nonexistent, but see [5] for a modest beginning. So, it is universally recognized that tests of methods on difficult problems are essential.

Invariably, any published proposal for a new numerical method will include some actual calculations, and these are clearly too numerous for us to catalogue. The book by Pat Roache [6] contains many early references, and the many fine texts now available (for example, [[, 8$]$ ) also contain references to calculations. Our concern here is to compare the behavior of some methods to each other on problems that seem to us to be sufficiently difficult and representative to enable the reader to draw some conclusions about the applicability of these methods. The now classic work of this nature is the paper by Gary Sod [9]. It showed up the shortcomings of schemes such as Lax-Wendroff and Lax-Friedrichs, and was very influential in the development of new methods. The one dimensional Riemann problem used by Sod in his tests is widely known as Sod's problem. Although it does show the ability of a method to resolve a rarefaction, a contact, and a shock, these waves in Sod's problem are not particularly strong.

A more difficult set of one-dimensional problems has been considered by E. Toro [110], and in that book Toro describes in detail several popular methods and shows their behavior on his tests all of which have easily computed exact solutions. We have included five of Toro's test problems, but we have gone beyond those to include some interesting two-dimensional tests, including one from [10]. In so doing, however, except in one case we no longer have exact solutions available, so a definitive objective evaluation of the validity of the solutions obtained is not possible.

Lagrangian methods, finite element methods, particle methods, kinetic and relaxation methods are not considered, nor are other systems of equations such as magneto-hydrodynamics.

OUTLINE: A detailed self-contained discussion of the ten schemes we have chosen would be impractical for this already rather large report, therefore in the next section we present only very briefly the basic ideas and references. Following that are three groups of tests. The first group consists of seven 1D Riemann problems plus the Woodward-Collela blast wave problem. The second group contains six 2D Riemann problems. The third group includes several 2D problems with unstable interfaces, plus one with an infinite strength shock. For each group the data are given, and then for each test in the group the output of the ten methods is collected. We also include some comments about the behavior of the various schemes. In those cases for which we do not have the exact solution, the comments are highly subjective. 
DISCLAIMER: Modifications had to be made to fit the various codes into our data structure. Bugs are always a possibility in that case, so we cannot guarantee that all schemes are functioning exactly as intended by their creators.

\section{$2 \quad$ Finite difference schemes}

Here we provide a short summary and references for all schemes used in this comparision project. We have chosen 10 methods that we feel are representative of the different basic finite difference approaches to solving hyperbolic conservation laws.

Two of the methods described below are dimensionally split, namely, PPM and WAFC. While we have chosen to describe all methods in only the most general terms, it is necesary to expound a bit here on the notion of dimensional splitting. Some have described this technique as ill-advised and inefficient, but we have found just the opposite to be the case, as have its defenders. For a system $u_{t}+f_{x}+g_{y}=0$ the time step is split into two parts. In the first, the equation $u_{t}+f_{x}=0$ is advanced by a one-dimensional scheme. Then using the updated values as data the equation $u_{t}+g_{y}=0$ is advanced to complete the time step. Either alternation or symmetrization as first proposed by G. Strang [1T] is usually used to preserve the accuracy of the 1D method and reduce grid alignment effects. On the other hand, the typical two-dimensional scheme would use difference approximations to $f_{x}$ and $g_{y}$ and simultaneously rather than sequentially update the data.

There are several advantages to dimensional splitting. It is very easy to convert a $1 \mathrm{D}$ code to $2 \mathrm{D}$ this way. The stability condition is usually less restrictive and there are possibly fewer flux evaluations necessary so that it can be more efficient than a $2 \mathrm{D}$ calculation. The big disadvantage is that it is not an option for non-rectangular grids.

\subsection{A composite scheme - CFLF}

This is the only first order scheme in this collection. In several papers on shallow water and the Euler equations, even in Lagrangian coordinates or on triangular grids [12, 13, 14, 15, 116, 177, 18] it was found that an effective method, called LWLFn, could be achieved by using a cycle consisting of $n-1$ time steps of some version of the Lax-Wendroff (LW) scheme followed by one step with Lax-Friedrichs (LF). The LF step acts as a consistent (with the differential equations) filter to reduce the oscillations of LW. Typically LWLF4 works best, but in some cases it is possible to take $n$ much larger. E. Toro pointed out to us that in 1D LWLF2 is the same as his FORCE scheme, [II0].

LWLFn is formally first-order, but optimally stable and symmetric for the $2 \mathrm{D}$ versions of LW and LF we have used. A full description of the final composite, called CFLF, is in [I2].

\subsection{A CFLF hybrid scheme - CFLFh}

Hybrid schemes and the similar flux-corrected-transport schemes have a long history and are presented very well in [8]. The idea is to create a numerical flux consisting of an average of a diffusive flux such as from Lax-Friedrichs (LF) and an oscillatory flux such as Lax-Wendroff (LW). The weights are chosen so that the scheme is formally second-order accurate but becomes sufficiently dissipative in shocks. We have used the fluxes from the LW and LF versions used in the composite scheme in subsection 2.1, along with the Harten weight [1.9] which can be found in $[8]$. 


\subsection{Centered scheme with limiter - JT}

This scheme by Guang-Shan Jiang and Eitan Tadmor [20] (which is the 2D successor of the Nessyahu-Tadmor 1D NT scheme [2] ] is called a nonoscillatory central scheme. It uses neither dimensional splitting nor eigenvector decomposition nor any overt Riemann solver. It does use discontinuous limited piecewise linear reconstruction from cell averages to get fluxes at cell edges.

The code is simple enough that it is presented in Appendix 5 of [20] and we were able to use it as is, making only those modifications necessary to fit it into our data structure.

\subsection{Positive scheme - LL}

This method devised by Xu-Dong Liu and Peter Lax (LL) [22, 2:3] is based on a theorem of Friedrichs stating roughly that if a finite difference method is a two-level method giving the new value of the solution vector as a linear combination of values at the previous time with coefficients that are positive symmetric matrices adding to the identity (but depending only on the independent variables), then the scheme is $L^{2}$ stable. The theorem doesn't apply directly to nonlinear systems, nevertheless Lax and Liu created such a positive scheme for the Euler equations. It does require an eigenvector decomposition and limiting.

We use the code for this scheme published in [22], available also electronicaly.

\subsection{Clawpack wave propagation scheme - CLAW}

Clawpack is a sophisticated flux splitting scheme developed by Randall LeVeque [24], based on earlier advection ideas [25]. The source and documentation are available to all at [26]. It has many options for the user; dimensionally split or not, choices for limiters, etc. We have used the nonsplit version with monotonized centered limiter using the Roe Riemann solver with 4 waves (separate shear and entropy waves).

\subsection{Weighted average flux (WAF) schemes - WAFT and WAFC}

WAF is actually a class of schemes that include the Roe scheme - a fact communicated to us by James Quirk [27, 28].

The flux at the cell boundary is obtained as a spatial weighted average over the states of an approximate Riemann solver. A limiter is employed in the computation of the weights. Different methods are obtained for different solvers and different averaging, [2.9, 10, 30]. We have used two versions; the first, WAFT, is a 2D nonsplit code given us by E. Toro and which is a part of the Numerica library [3]]. This code uses a WAF scheme with HLLC approximate Riemann solver using the Rankine-Hugoniot condition for evaluating the middle fluxes as described in chapter 10 of [10].

The other is a dimensionally split version, which we call WAFC, similar to WAFT, but using the HLLC approximate Riemann solver with Einfeldt [32] speeds, and fluxes evaluated at the middle states.

\subsection{Weighted essentially nonoscillatory schemes - WENO5 and CWENO3}

Weighted essentially nonoscillatory schemes (WENO) [33] are an improvement on the essentially nonoscillatory (ENO) scheme of Harten and Osher [34]. Upwind biased spatial differencing is used that produces high order accuracy for smooth flows but becomes low order and dissipative 
for shocks (a simple description of the basis of the WENO3 method has been presented in [12]). We have used two versions of WENO; in one (WENO5) the WENO procedure is applied to an eigenvector decomposition and is fifth-order accurate, the other we call CWENO3 (C comming from conserved variables), in which the upwinding is done on the conserved variables directly and is third-order accurate. The time integration is done with Runge-Kutta.

For WENO5 we use the code given to us by Guan-Shan Jiang with RK3 time integration and for CWENO3 our code with RK3 time integration as described in [12].

\subsection{Piecewise parabolic - PPM}

The piecewise parabolic method (PPM) [35, 36] is in the class of higher order accurate Godunov methods. It uses piecewise parabolic limited reconstruction to obtain states to use in the Riemann problems defining the fluxes. Dimensional splitting is used.

We use the free version of PPM available at PPMLib library [37] (core routines are available only as SGI binaries). 


\section{1D tests}

\subsection{Description of 1D problems}

For 1D tests we have chosen five 1D (in $x$ ) Riemann problems from [10], tests 1,2,4,5,6 plus four others: test 1-tvj is the same as test 1 but with a large jump in the y-velocity; Noh is the classical 1D Noh problem [38]; test $3 a$ is a modification of test 3 from [10] keeping a stationary contact; peak is a hard problem with strong narrow peak in density found by Milan Kuchařík [3.9]), and the Woodward-Collela blast wave problem [35]. All the 1D problems except the blast wave problem are simple Riemann problems with known exact solutions.

All codes are 2D-capable, that is, they have two velocity components. The Riemann problems are on the interval $x \in(0,1)$ (except for peak which is computed on $x \in(0.1,0.6)$ ) with initial discontinuity at $x_{0} \in(0,1)$ solved for time $t \in(0, T)$. The initial conditions are given by constant left state $\left(\rho_{L}, u_{L}, v_{L}, p_{L}\right)$ of density, $x$-velocity, $y$-velocity, and pressure on the interval $x \in\left(0, x_{0}\right)$ and right state $\left(\rho_{R}, u_{R}, v_{R}, p_{R}\right)$ on the interval $x \in\left(x_{0}, 1\right)$. Each test is defined by the ten parameters $\rho_{L}, u_{L}, v_{l}, p_{L}, \rho_{R}, u_{R}, v_{R}, p_{R}, x_{0}, T$. For all 1D Riemann problems except test 1-tvj, the data are given in Table 1, together with $v_{L}=v_{R}=0$. The data for test 1-tvj is the same as for test test 1 , together with $v_{L}=1, v_{R}=-5$. The Noh problem uses the gas constant $\gamma=5 / 3$ while all other tests use $\gamma=1.4$. All Riemann problem tests use natural boundary conditions.

\begin{tabular}{|l|ccc|ccc|cc|}
\hline Test & $\rho_{L}$ & $u_{L}$ & $p_{L}$ & $\rho_{R}$ & $u_{R}$ & $p_{R}$ & $x_{0}$ & $T$ \\
\hline 1 & 1 & 0.75 & 1 & 0.125 & 0 & 0.1 & 0.3 & 0.2 \\
2 & 1 & -2 & 0.4 & 1 & 2 & 0.4 & 0.5 & 0.15 \\
Noh & 1 & 1 & $10^{-6}$ & 1 & -1 & $10^{-6}$ & 0.5 & 1 \\
$3 \mathrm{a}$ & 1 & -19.59745 & 1000 & 1 & -19.59745 & 0.01 & 0.8 & 0.012 \\
4 & 5.99924 & 19.5975 & 460.894 & 5.99242 & -6.19633 & 46.095 & 0.4 & 0.035 \\
5 & 1.4 & 0 & 1 & 1 & 0 & 1 & 0.5 & 2 \\
6 & 1.4 & 0.1 & 1 & 1 & 0.1 & 1 & 0.5 & 2 \\
peak & 0.1261192 & 8.9047029 & 782.92899 & 6.591493 & 2.2654207 & 3.1544874 & 0.5 & 0.0039 \\
\hline
\end{tabular}

Table 1: Definition of 1D Riemann problem tests

The classic Woodward-Collela blast wave problem [35] computes the interaction of waves from two Riemann problems with reflecting boundary conditions. The problem is treated again on the interval $x \in(0,1)$. Two initial discontinuities are located at $x_{1}=0.1$ and $x_{2}=0.9$. The initial density is one and the velocity is zero everywhere. Initial pressures in three different regions (left $p_{l}$, middle $p_{m}$ and right $\left.p_{r}\right)$ are $\left(p_{l}, p_{m}, p_{r}\right)=(1000,0.01,100)$.

For the numerical treatment of most test problem we use 100 grid cells, exceptions being tests $3 a$ and 4 using 200 cells, blast using 400 and 2000 cells and peak using 800 cells. 


\subsection{Errors of the numerical solution}

For 1D Riemann problems we can compute their exact solution and so we are able to compare the errors of their numerical solution, giving us an objective evaluation of the different numerical methods. Table 2 summarizes $L_{1}, L_{2}$ and maximum relative errors of the numerical solution of these 1D Riemann problems by different numerical schemes. Errors are in \%. For most tests presented errors are errors in density, only for test 2 we present errors in internal energy and for peak in velocity.

\begin{tabular}{|c|c|c|c|c|c|c|c|c|c|}
\hline Test & 1 & 1-tvj & 2 & noh & $3 \mathrm{a}$ & 4 & 5 & 6 & peak \\
\hline & \multicolumn{9}{|c|}{ relative $L^{1}$ errors } \\
\hline CFLF & 2.0 & 7.3 & 16.4 & 1.4 & 12.0 & 3.8 & 1.5 & 0.7 & 3.0 \\
\hline CFLFh & 1.5 & 8.1 & 10.2 & 1.9 & 10.3 & 2.7 & 0.7 & 0.8 & 1.9 \\
\hline WAFT & 0.7 & 3.3 & 21.9 & 2.8 & 2.6 & 1.4 & 0 & 0.3 & 1.0 \\
\hline WAF4 & 0.8 & 7.8 & fail & 1.4 & 2.8 & 1.4 & 0 & 0.3 & 1.1 \\
\hline CLAW & 0.8 & 5.5 & fail & 1.3 & 3.1 & 1.7 & 0 & 0.4 & fail \\
\hline PPM & 0.5 & 3.9 & 6.3 & 4.6 & 9.4 & 1.1 & 0 & 0.1 & 1.3 \\
\hline WENO & 1.3 & 3.5 & 23.7 & 2.0 & 9.2 & 2.2 & 0 & 0.4 & 2.4 \\
\hline CWENO & 2.2 & 6.8 & 28.2 & 2.5 & 14.7 & 3.9 & 1.1 & 1.1 & 2.4 \\
\hline LL & 1.3 & 10.0 & 31.3 & 1.5 & 5.2 & 2.4 & 0.5 & 0.7 & 0.8 \\
\hline \multirow[t]{2}{*}{$\mathrm{JT}$} & 1.3 & 3.9 & 6.4 & 1.7 & 8.1 & 2.3 & 0.6 & 0.6 & 1.1 \\
\hline & \multicolumn{9}{|c|}{ relative $L^{2}$ errors } \\
\hline CFLF & 3.6 & 11.8 & 22.4 & 6.0 & 31.5 & 10.4 & 3.7 & 2.4 & 7.0 \\
\hline CFLFh & 3.2 & 13.2 & 18.8 & 7.1 & 30.2 & 8.5 & 2.5 & 2.5 & 5.8 \\
\hline WAFT & 1.9 & 7.9 & 36.3 & 5.8 & 16.3 & 5.4 & 0 & 1.4 & 4.2 \\
\hline WAF4 & 1.9 & 13.5 & fail & 5.5 & 17.6 & 5.5 & 0 & 1.4 & 4.5 \\
\hline CLAW & 2.3 & 10.5 & fail & 5.9 & 18.2 & 6.5 & 0 & 1.8 & fail \\
\hline PPM & 1.5 & 8.4 & 9.7 & 11.1 & 31.6 & 4.7 & 0 & 0.7 & 5.1 \\
\hline WENO & 2.8 & 7.3 & 36.5 & 7.4 & 32.5 & 8.2 & 0 & 1.7 & 7.8 \\
\hline CWENO & 3.7 & 11.7 & 38.8 & 8.3 & 36.5 & 11.1 & 3.1 & 3.0 & 6.6 \\
\hline LL & 3.2 & 13.9 & 36.1 & 6.5 & 21.9 & 8.3 & 2.1 & 2.5 & 4.4 \\
\hline \multirow[t]{2}{*}{ JT } & 2.7 & 8.4 & 8.4 & 6.9 & 28.3 & 7.7 & 2.4 & 2.3 & 5.1 \\
\hline & \multicolumn{9}{|c|}{ relative $\max$ errors } \\
\hline CFLF & 11.0 & 31.2 & 33.7 & 34.5 & 56.9 & 25.3 & 13.2 & 14.2 & 71.2 \\
\hline CFLFh & 11.9 & 34.1 & 39.6 & 39.4 & 67.9 & 25.1 & 11.5 & 12.9 & 76.7 \\
\hline WAFT & 8.6 & 30.8 & 57.8 & 29.8 & 53.4 & 20.4 & 0 & 10.1 & 76.2 \\
\hline WAF4 & 8.9 & 43.1 & fail & 31.6 & 56.4 & 20.3 & 0 & 10.1 & 77.6 \\
\hline CLAW & 9.4 & 32.0 & fail & 33.1 & 56.4 & 22.8 & 0 & 11.4 & fail \\
\hline PPM & 6.2 & 23.3 & 22.7 & 51.3 & 71.3 & 15.4 & 0 & 4.7 & 80.3 \\
\hline WENO & 9.0 & 21.7 & 61.7 & 41.4 & 73.7 & 31.3 & 0 & 10.4 & 80.3 \\
\hline CWENO & 10.9 & 32.7 & 53.6 & 45.4 & 65.6 & 41.2 & 12.7 & 12.7 & 78.0 \\
\hline LL & 11.3 & 28.2 & 40.3 & 37.4 & 57.1 & 28.2 & 12.1 & 14.3 & 77.8 \\
\hline JT & 10.6 & 28.9 & 16.1 & 39.5 & 67.0 & 23.9 & 12.0 & 12.1 & 80.6 \\
\hline
\end{tabular}

Table 2: Relative $L^{1}, L^{2}$ and $\max$ errors in $\%$ for 1D Riemann problem tests for all 10 schemes, fail means that the scheme has failed to compute given test. 


\section{$3.31 \mathrm{D}$ results}

Results are shown in the following subsections and figures.

All codes are 2D-capable, that is, both velocity components are advanced. In all 1D problems the transverse velocity is 0 , except Test 1 -tvj.

Note that for most problems we present the results for the density. The exception are the test 2 for which we present internal energy and peak for which we present velocity and density.

Test 1: This is Toro's variant of Sod's Riemann problem, differing from it in that there is a sonic point in the rarefaction. All ten methods resolve the shock very well without oscillation with the exception of CFLF, although CWENO3 and LL are more dissipative than the others. PPM is outstanding on the contact, with WAFC and WAFT not far behind. Many of the schemes tested in [10] develop the so-called sonic glitch in the rarefaction, but this is not present in any of our ten schemes, but WAFC, PPM, CLAW, and JT have a dip at the base of the rarefaction wave.

Test 1-tvj: This has the same data as Test 1, but there is a large jump in transverse velocity, which should have no effect on $r h o, u$, or $p$. This produces an error in the kinetic energy and destroys the solution for all schemes. However, if we run WAFC for this problem with removed transverse kinetic energy then we get no effect from the nonconstant transverse velocity, obtaining the same result as for test 1 . This is an option only available to dimensionally split schemes and it works well in 2D only for jumps aligned with the grid. When we tried WAFC with the transverse kinetic energy removed for the 2D Noh problem (described later), it gives the wrong shock speed. For that problem, Paul Woodward [40] pointed out to us that conversion of kinetic energy to heat is the critical mechanism, therefore in that case ignoring some kinetic energy is not a good idea. But in [4]] the transverse kinetic energy removal is part of a scheme for two-fluid problems and seems to be very effective.

Test 2: For this Riemann problem the central state is a near vacuum, in which both $\rho$ and $p$ are close to zero, but the internal energy $e=p / \rho(\gamma-1)$ is not. It seems that no general Eulerian scheme can compute the internal energy very well. To make LL work for this problem we needed to set LL parameters to $\alpha=0.1$ and $\beta=2$ on Xu-Dong Liu's suggestion [42] which leads to larger viscosity and bad resolution of the heads of rarefaction waves .

1D-Noh: The solution of this problem consists of two infinite strength shocks moving out from the center, leaving a constant density and pressure state behind. CFLF has a slight overshoot at the shocks, but only a very small dip in density at the center, as does JT. PPM has a poorly resolved shock and a significant dip at the center, and is not symmetric about the center. The WAFT run was with the superbee limiter and is also not symmetric, while a run using minmod limiter was symmetric.

Test 3a: In this variant of Toro's Test 3 there is a stationary contact generated at $x=.8$. The WAF schemes and CLAW do best, with CFLF and CFLFhybrid not really acceptable at this resolution.

Test 4: PPM is very good on this problem with two strong shock waves. CLAW and both WAFs have good resolution but develop oscillations.

Test 5: This shows which methods (WAFT, WAFC, CLAW, PPM and WENO5) are exact for a stationary contact.

Test 6: This is a slowly moving contact. PPM is excellent, followed by WAFT, WAFC, CLAW and WENO5. CFLF and CFLFh develop oscillations behind the contact.

Peak: All schemes have difficulty accurately computing the velocity, especially around the rarefaction with very small change in density. WAFT and WAFC resolve very nicely the very narrow peak in density between the contact and the shock. 
Blast: For this test we present results computed using 400 cells (with "exact" solution from PPM with 2000 cells) and results computed using 2000 cells (with "exact" solution from PPM with 2000 cells). 


\section{Test 1}

\section{CFLF}
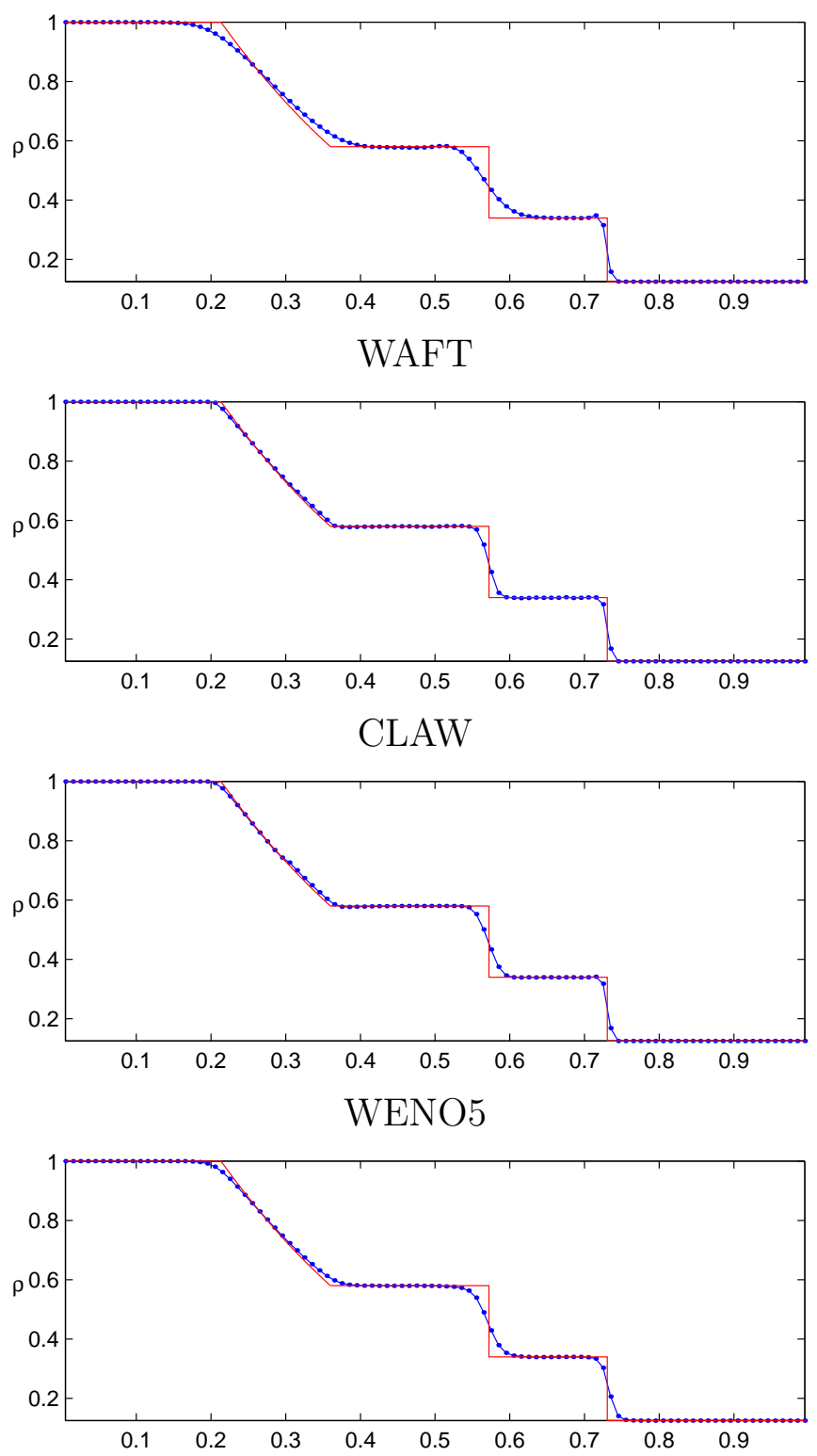

LL

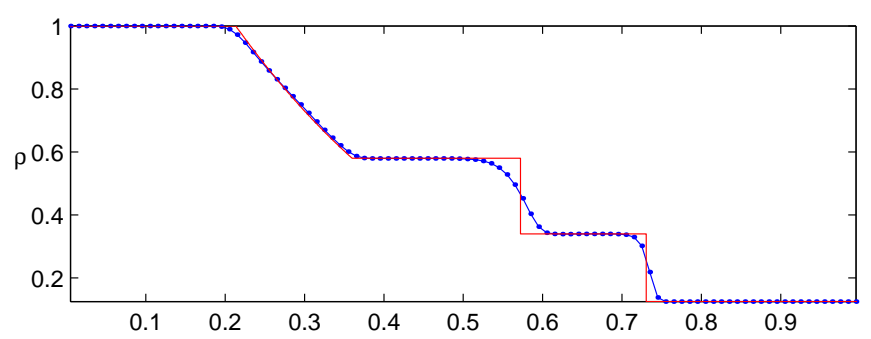

CFLFh
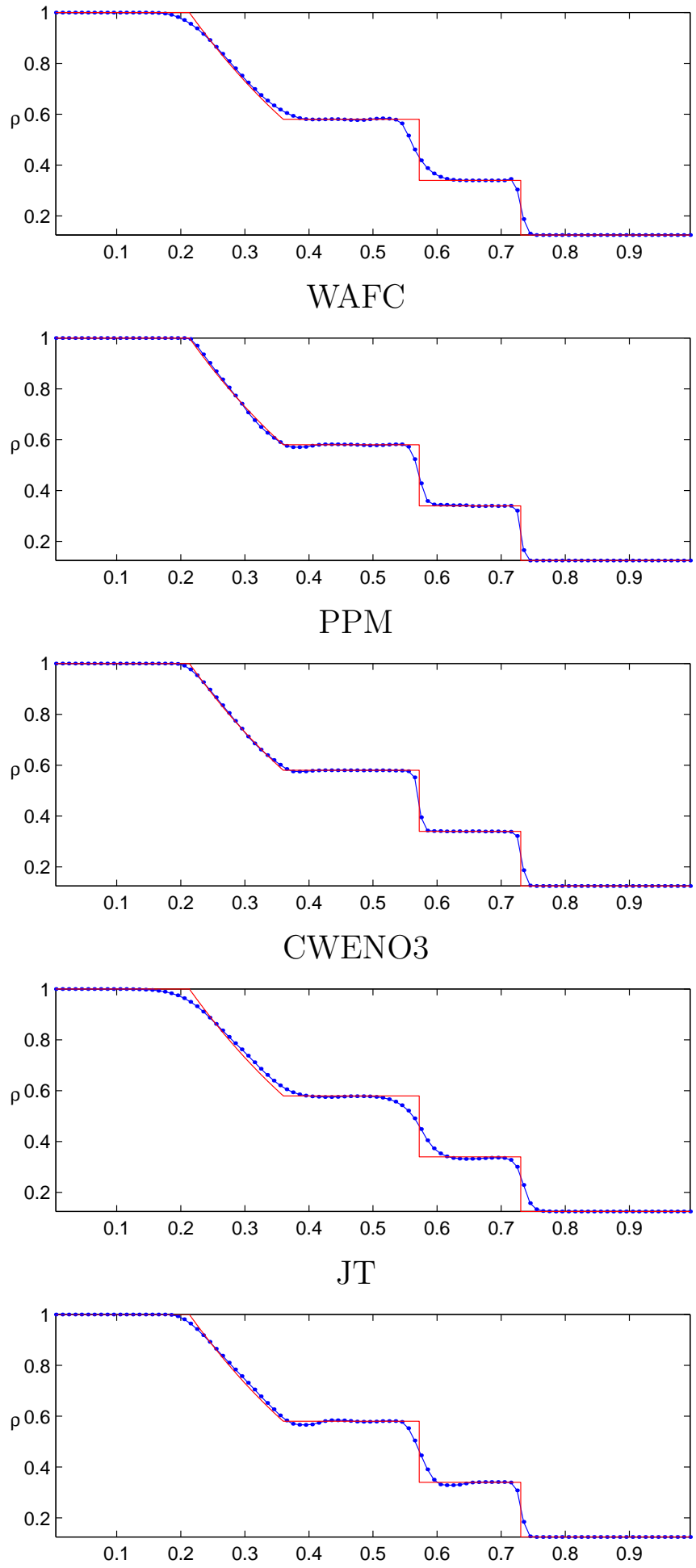

Figure 1: 1D results (density) for the test 1 problem by all ten schemes. 
Test 1 with jump in transverse velocity

\section{CFLF}
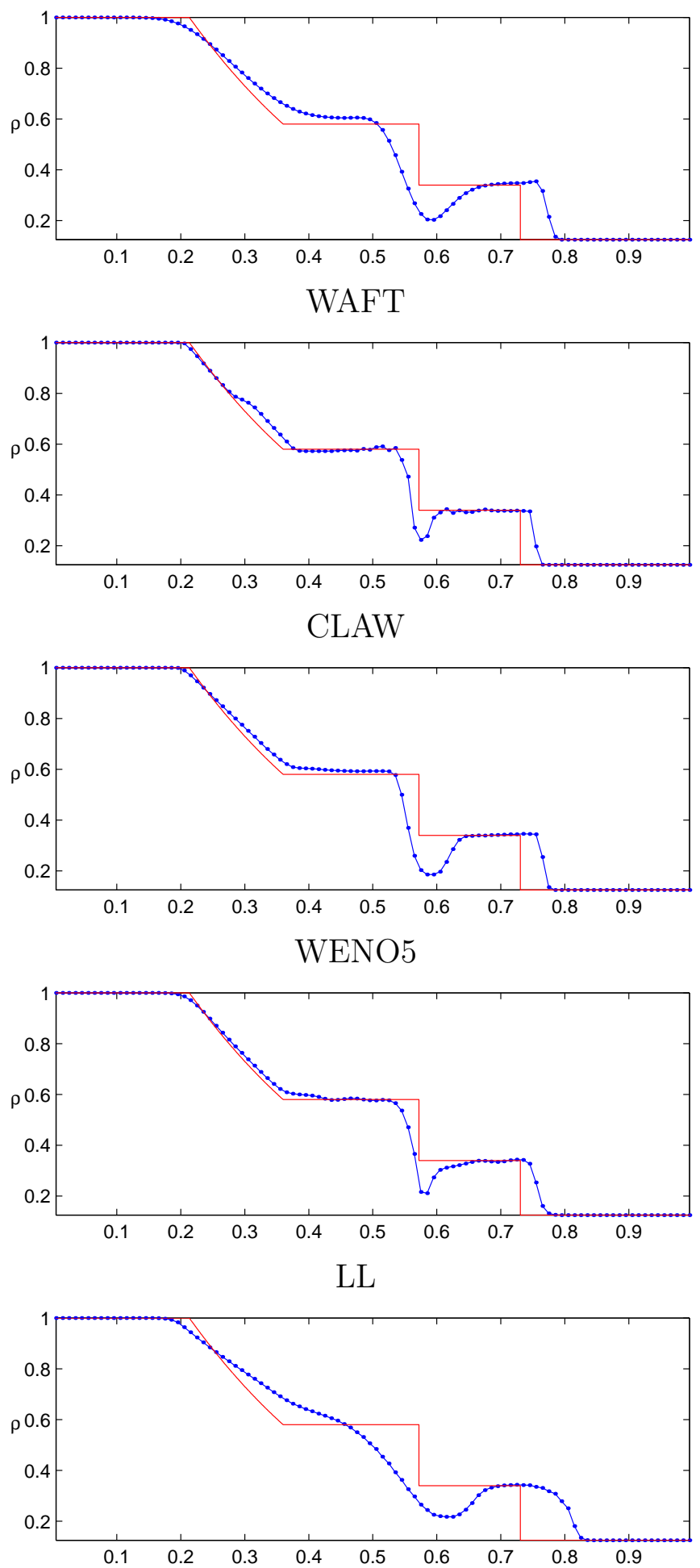

CFLFh
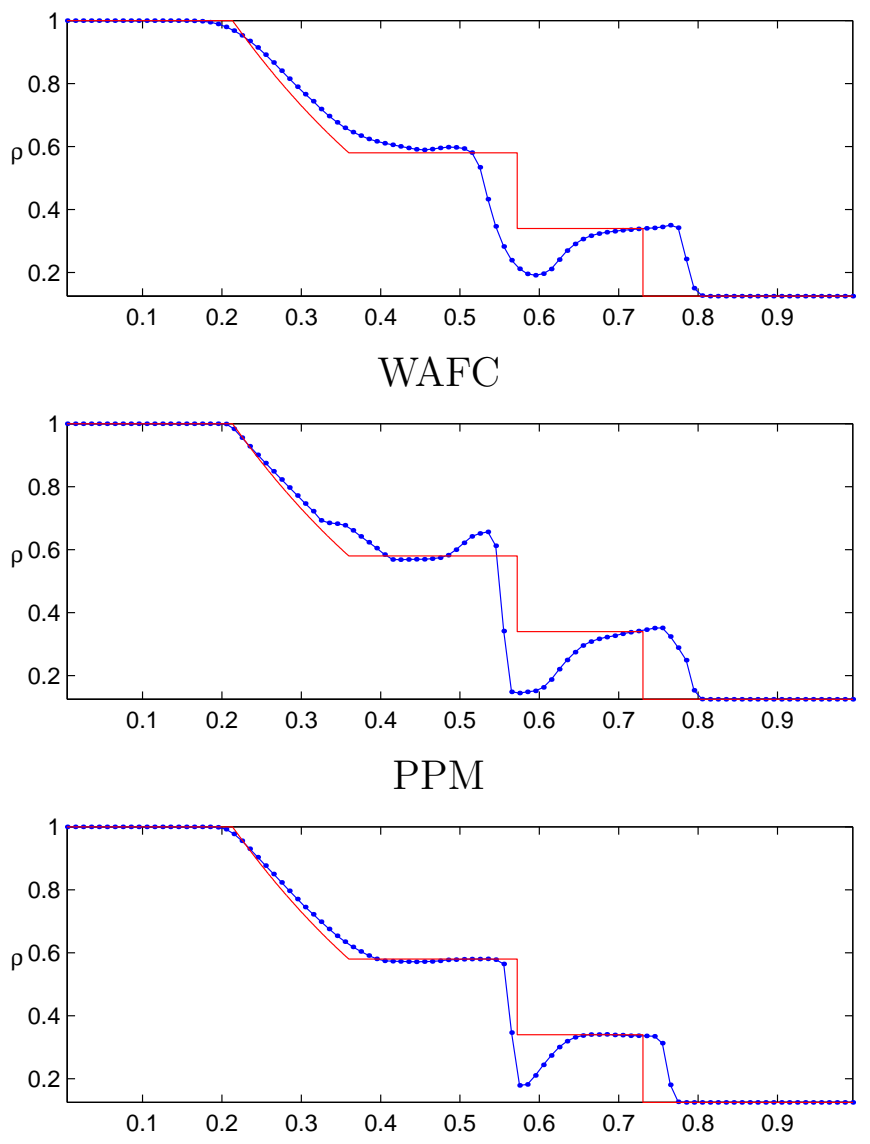

CWENO3
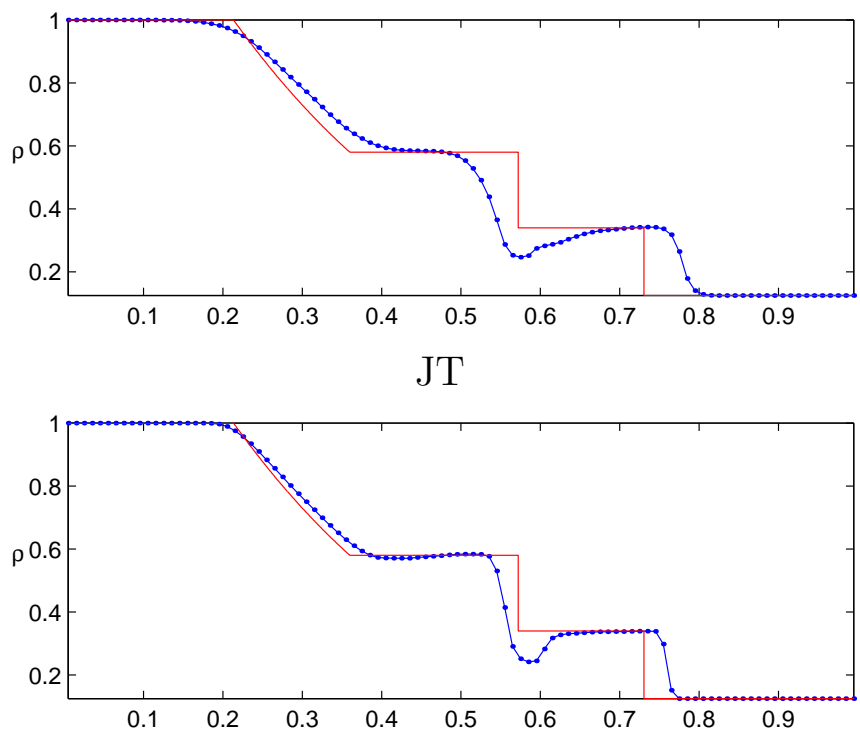

Figure 2: 1D results (density) for the test 1 with tvj problem by all ten schemes. 


\section{Test 2}

\section{CFLF}
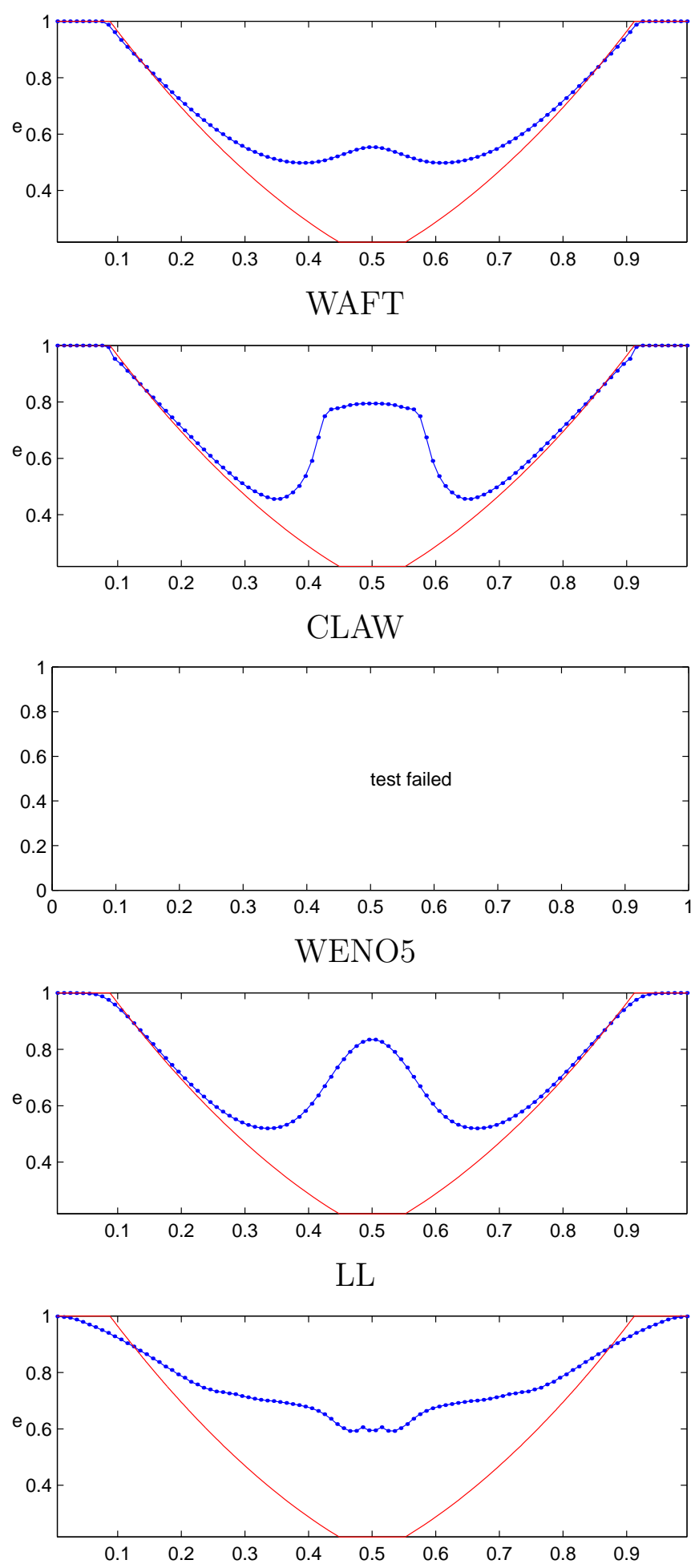

\section{$\mathrm{CFLFh}$}
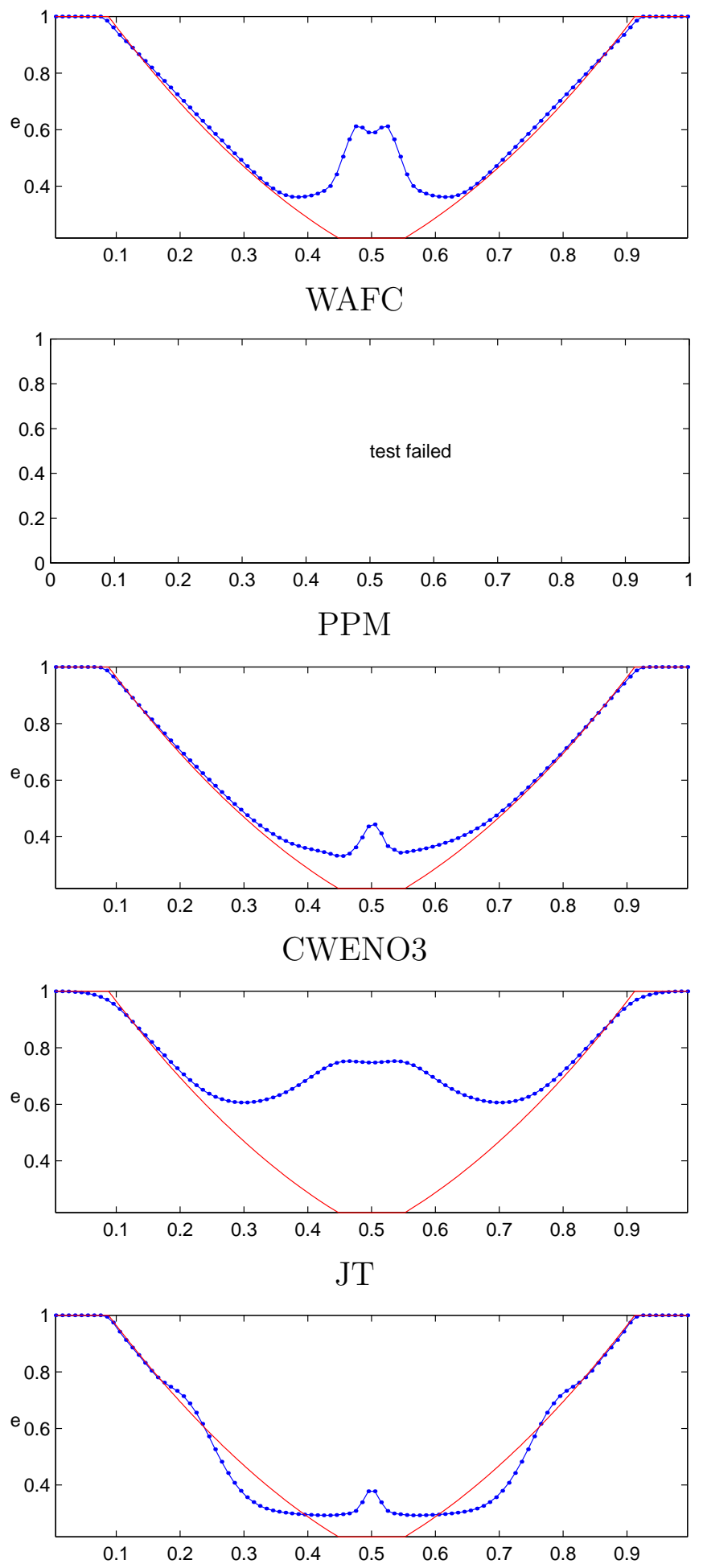

Figure 3: 1D results (internal energy) for the test 2 problem by all ten schemes. 


\section{CFLF}
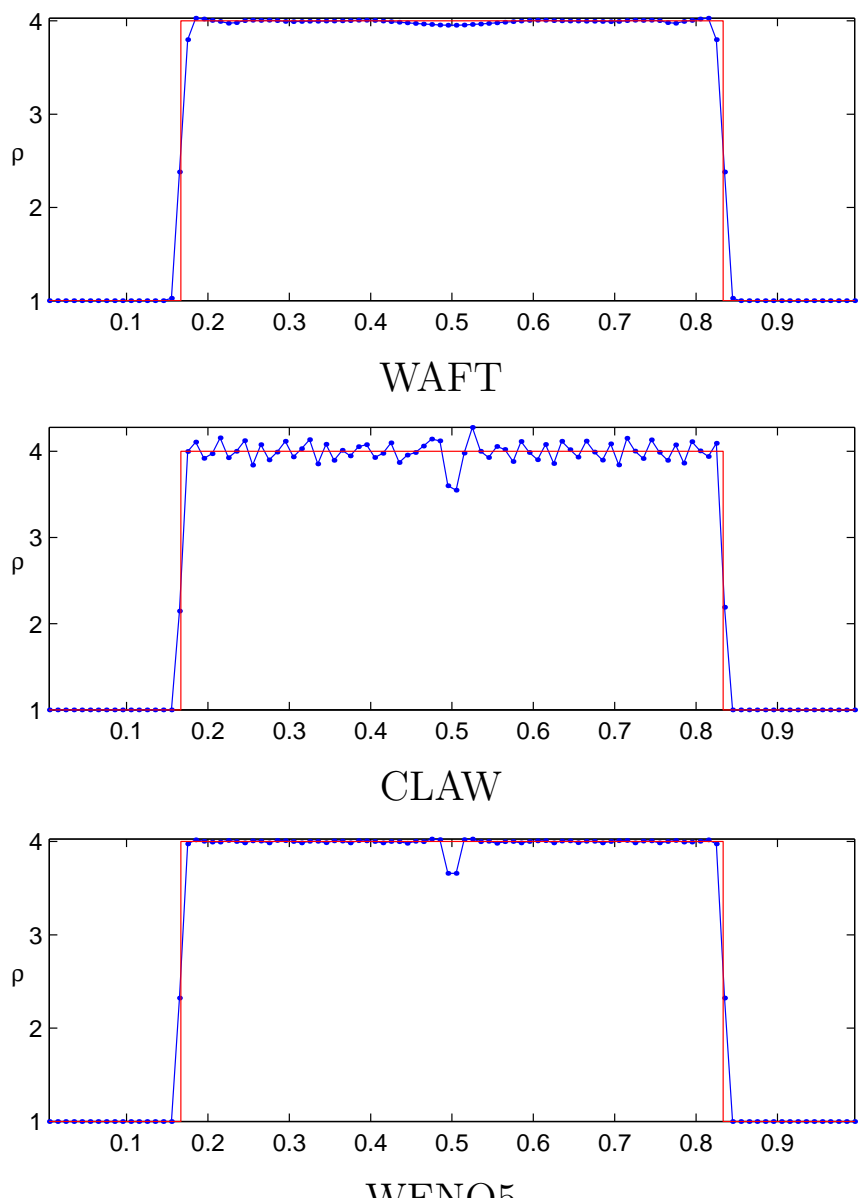

WENO5

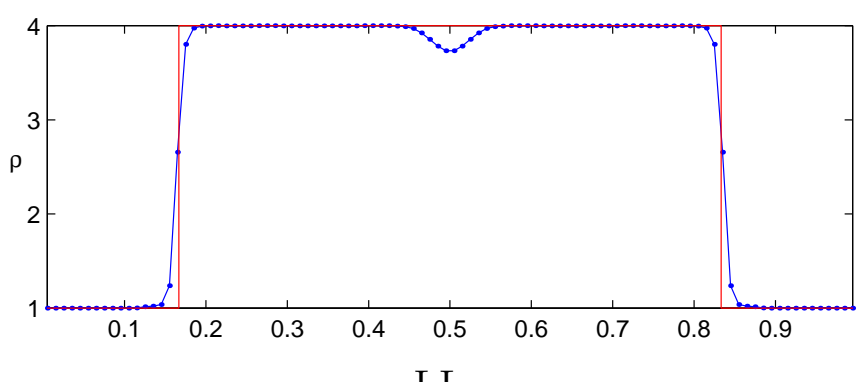

LL

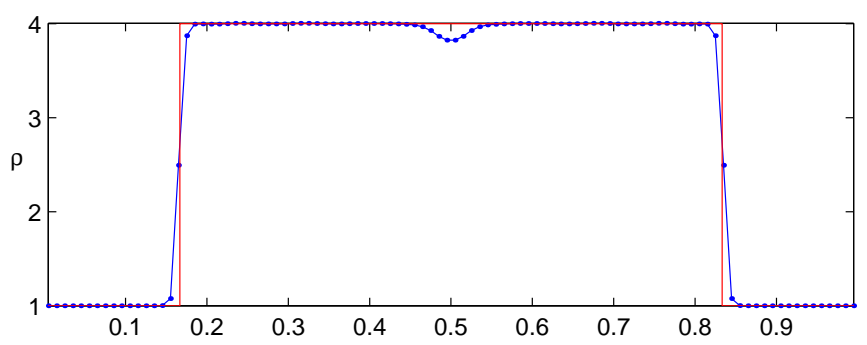

CFLFh
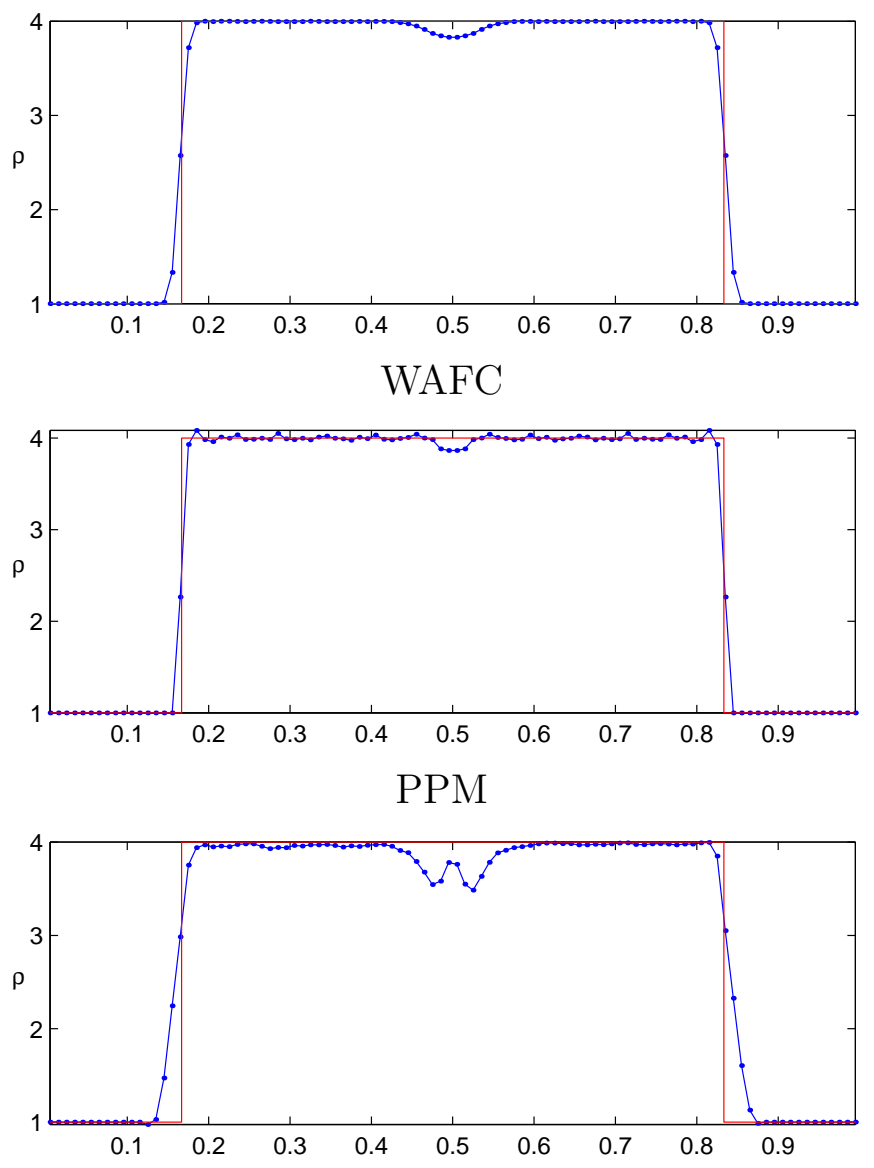

CWENO3
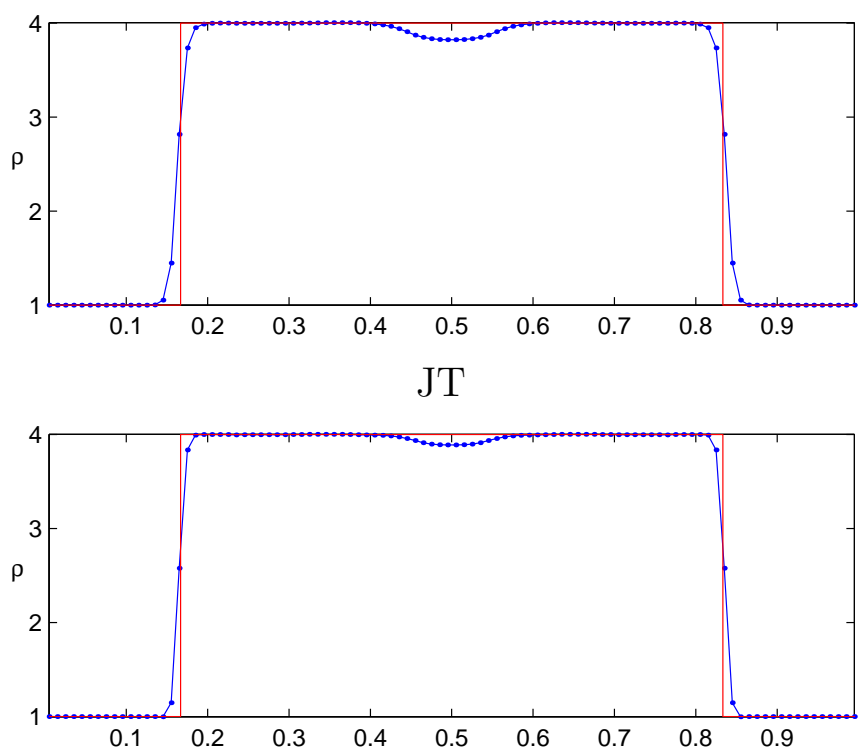

Figure 4: 1D results (density) for the Noh problem by all ten schemes. 


\section{Test 3 a}

CFLF
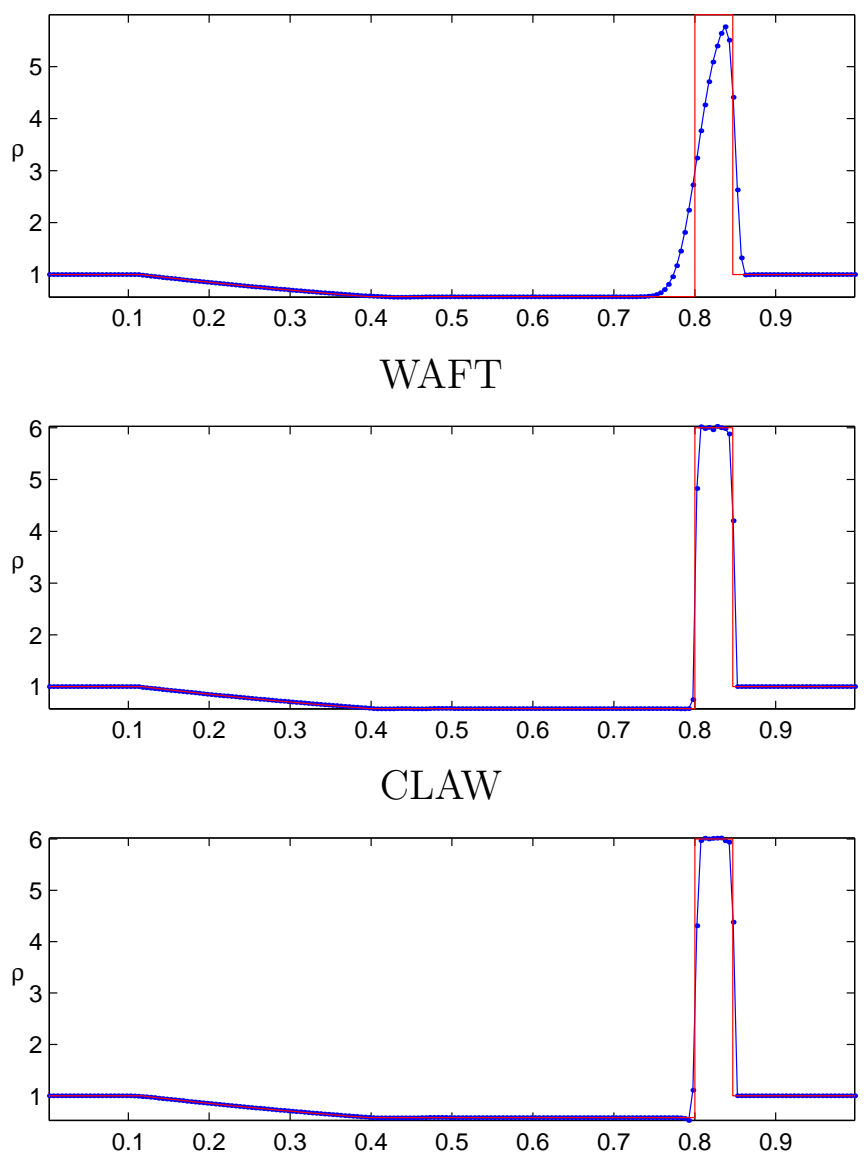

WENO5

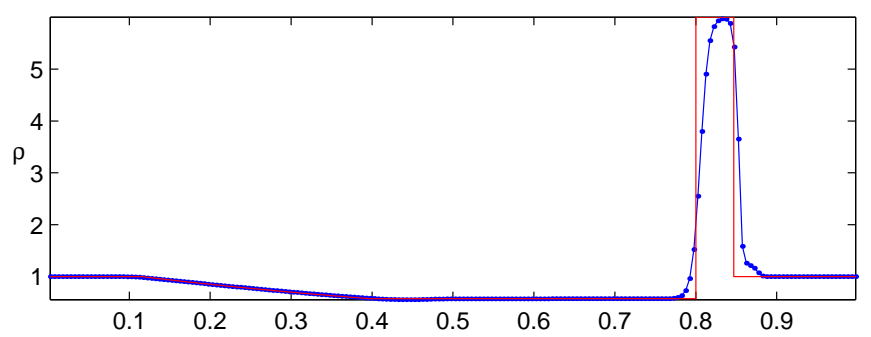

LL

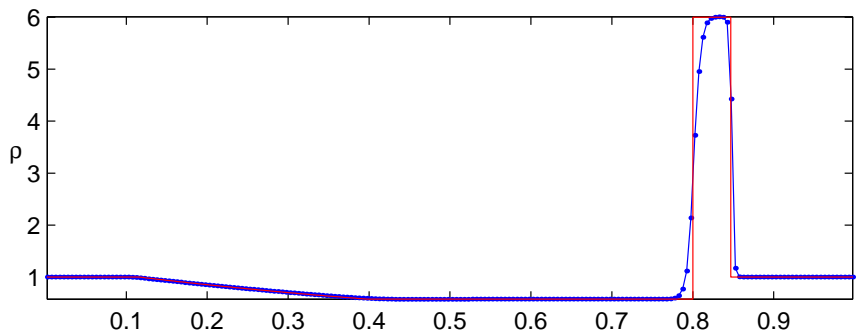

CFLFh
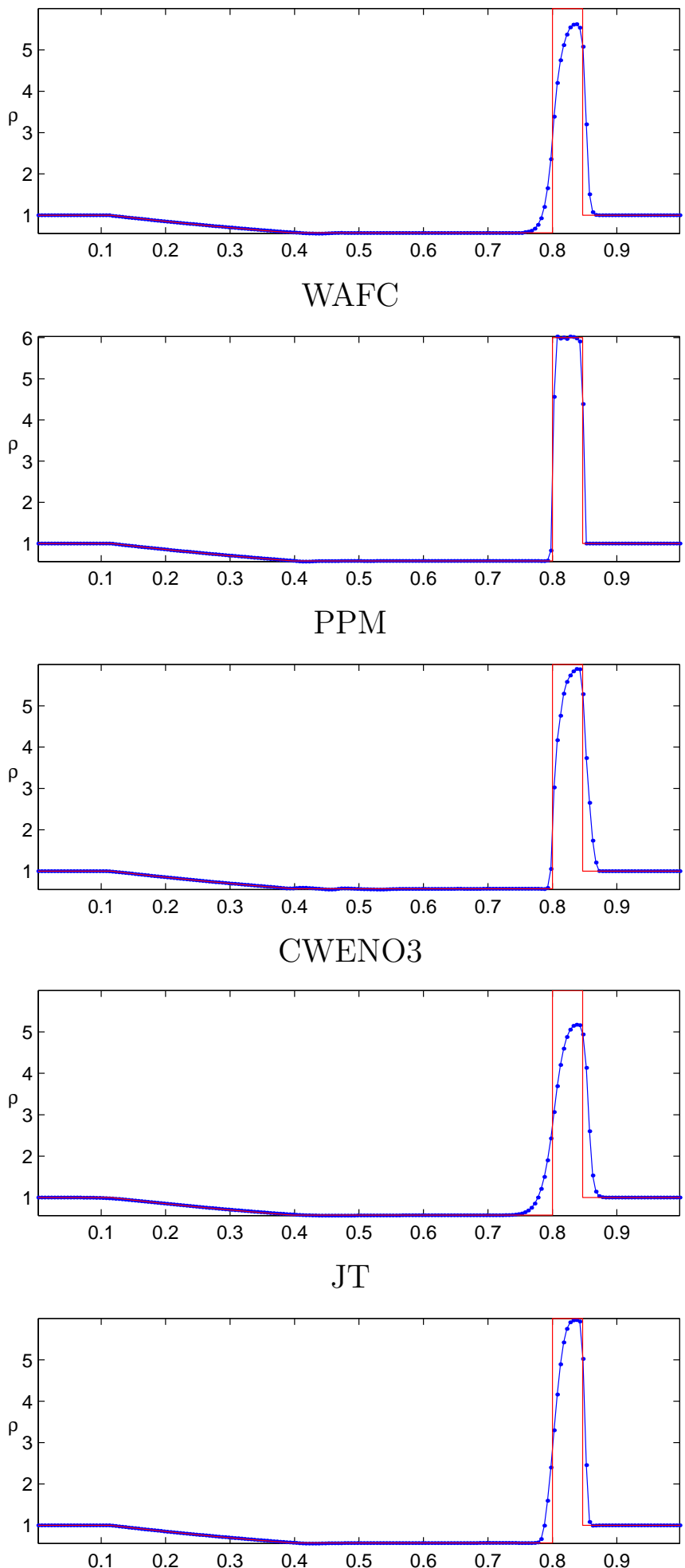

Figure 5: 1D results (density) for the test 3 a problem by all ten schemes. 


\section{Test 4}

\section{CFLF}
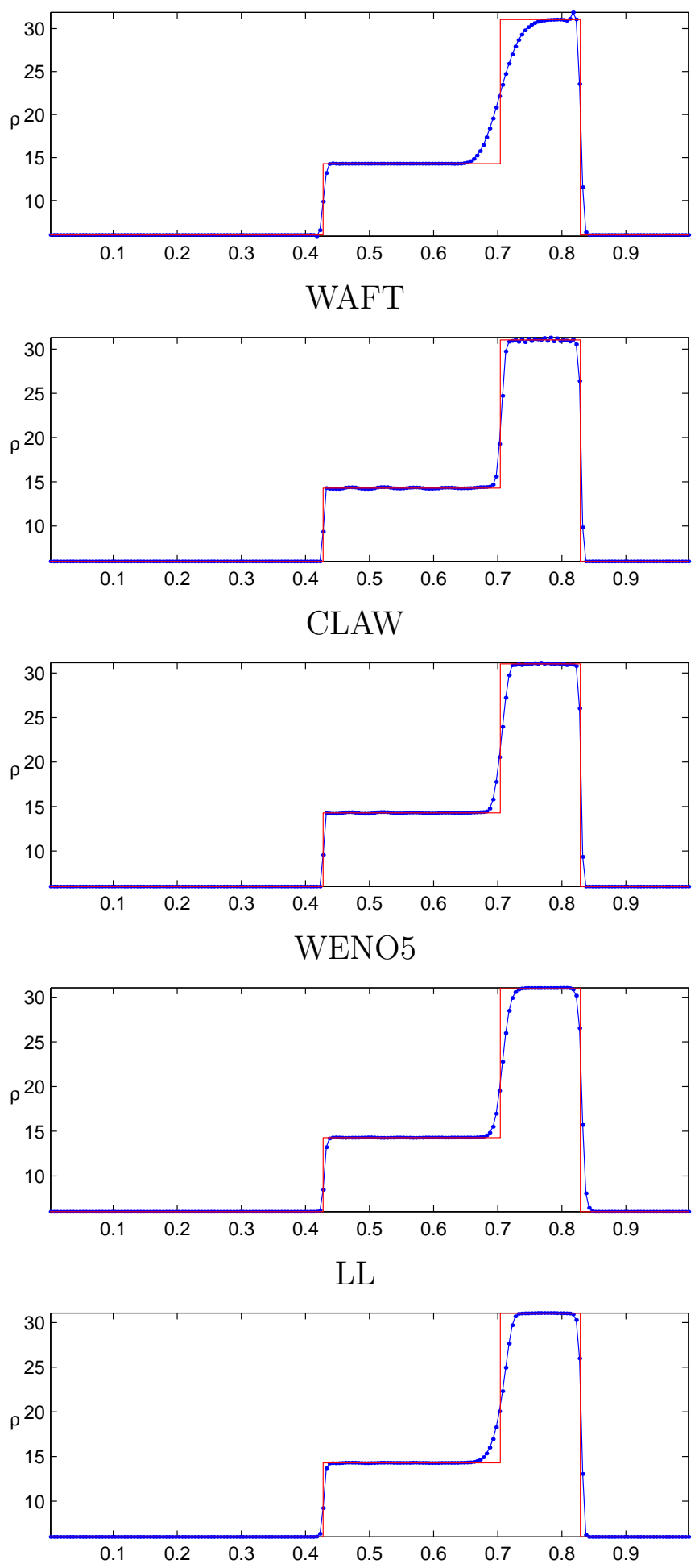

CFLFh
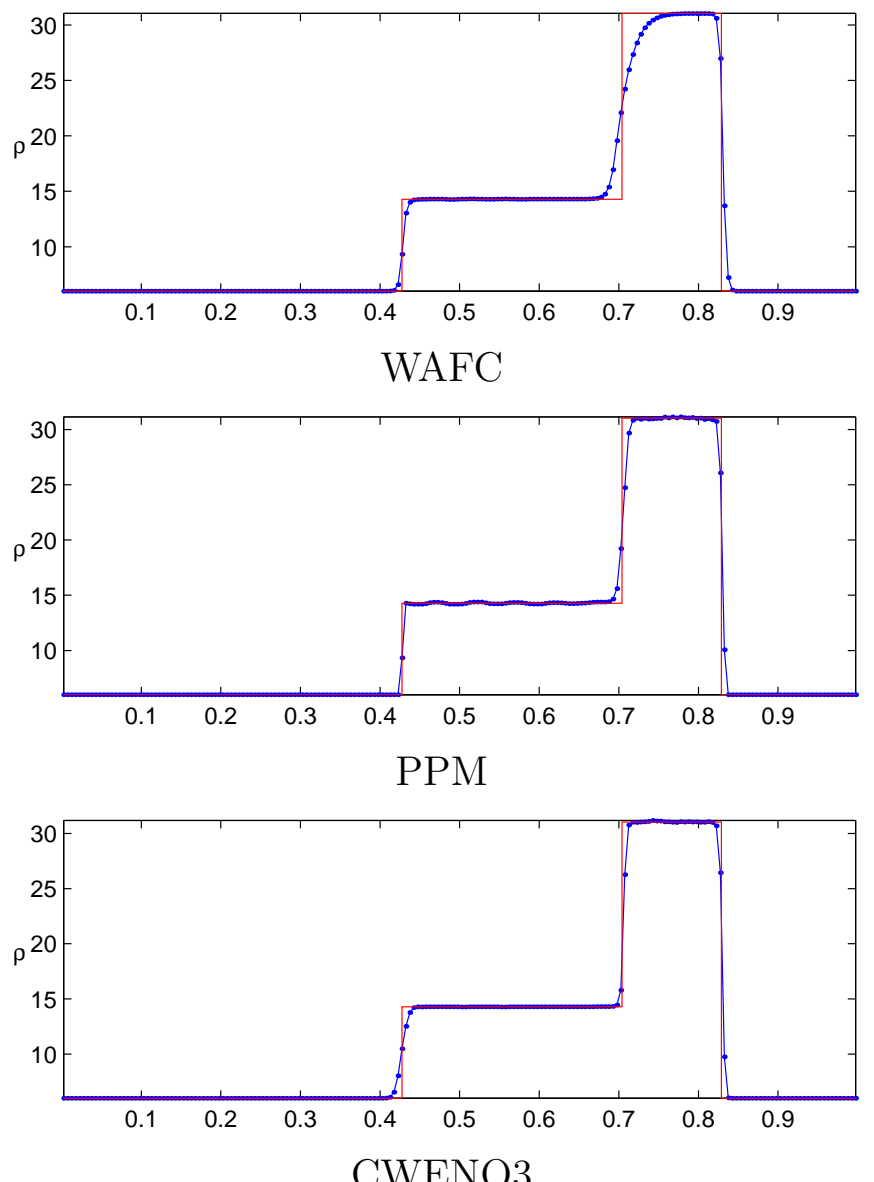

CWENO3
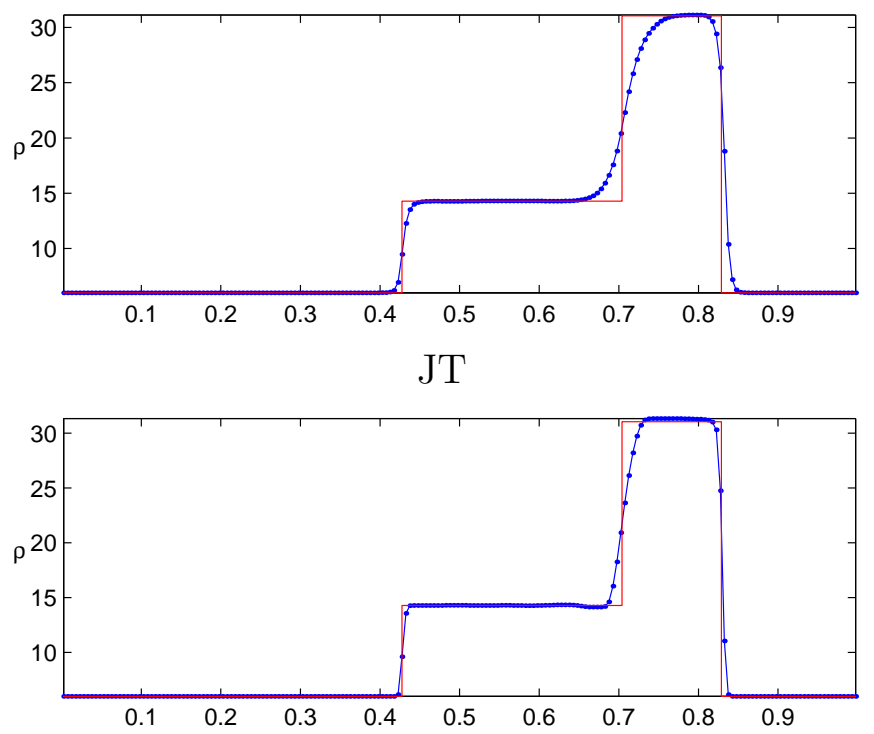

Figure 6: 1D results (density) for the test 4 problem by all ten schemes. 


\section{Test 5}

\section{CFLF}
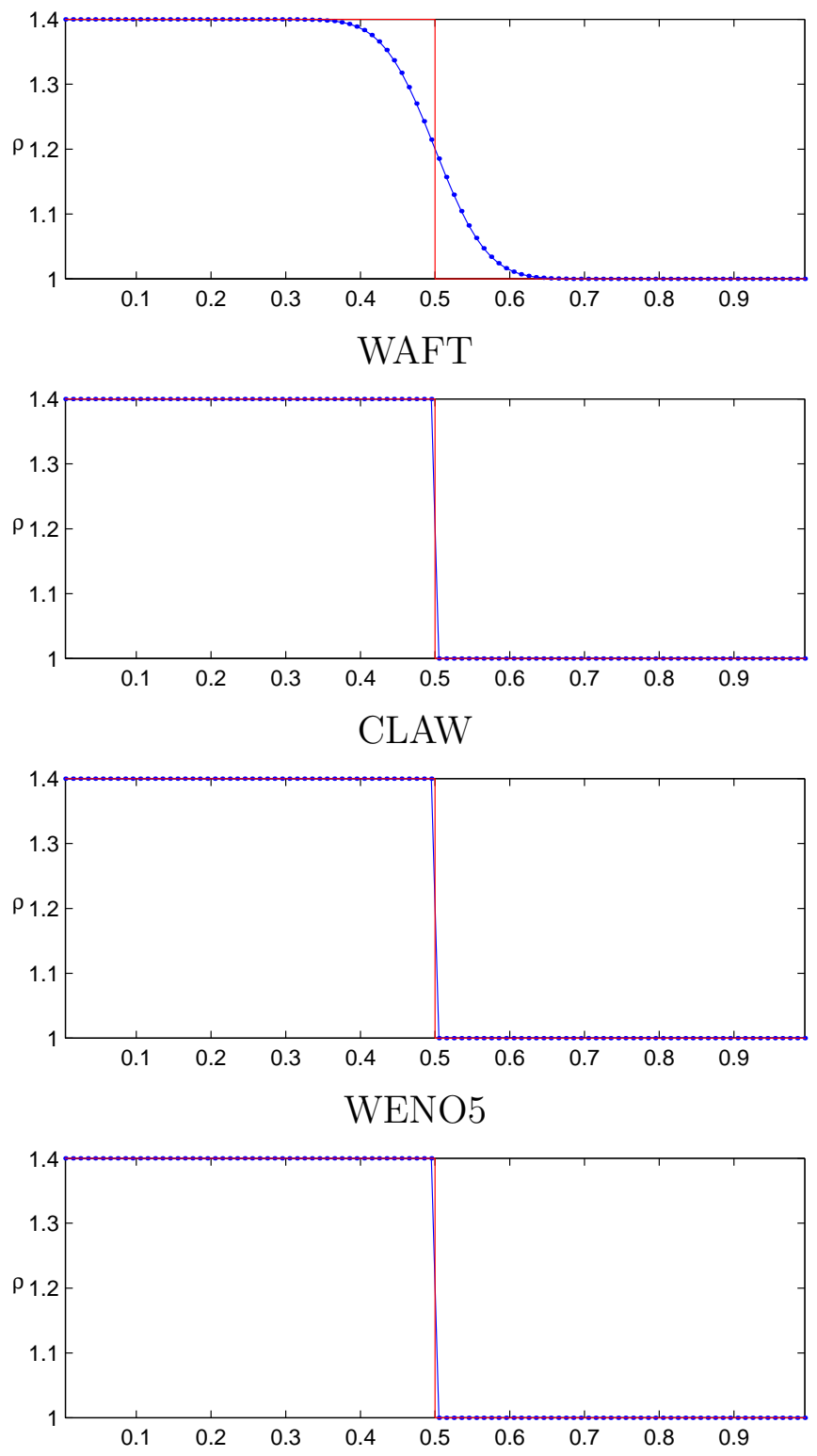

LL

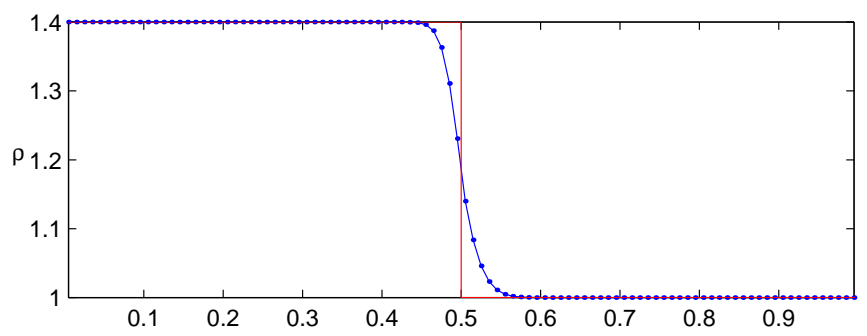

CFLFh
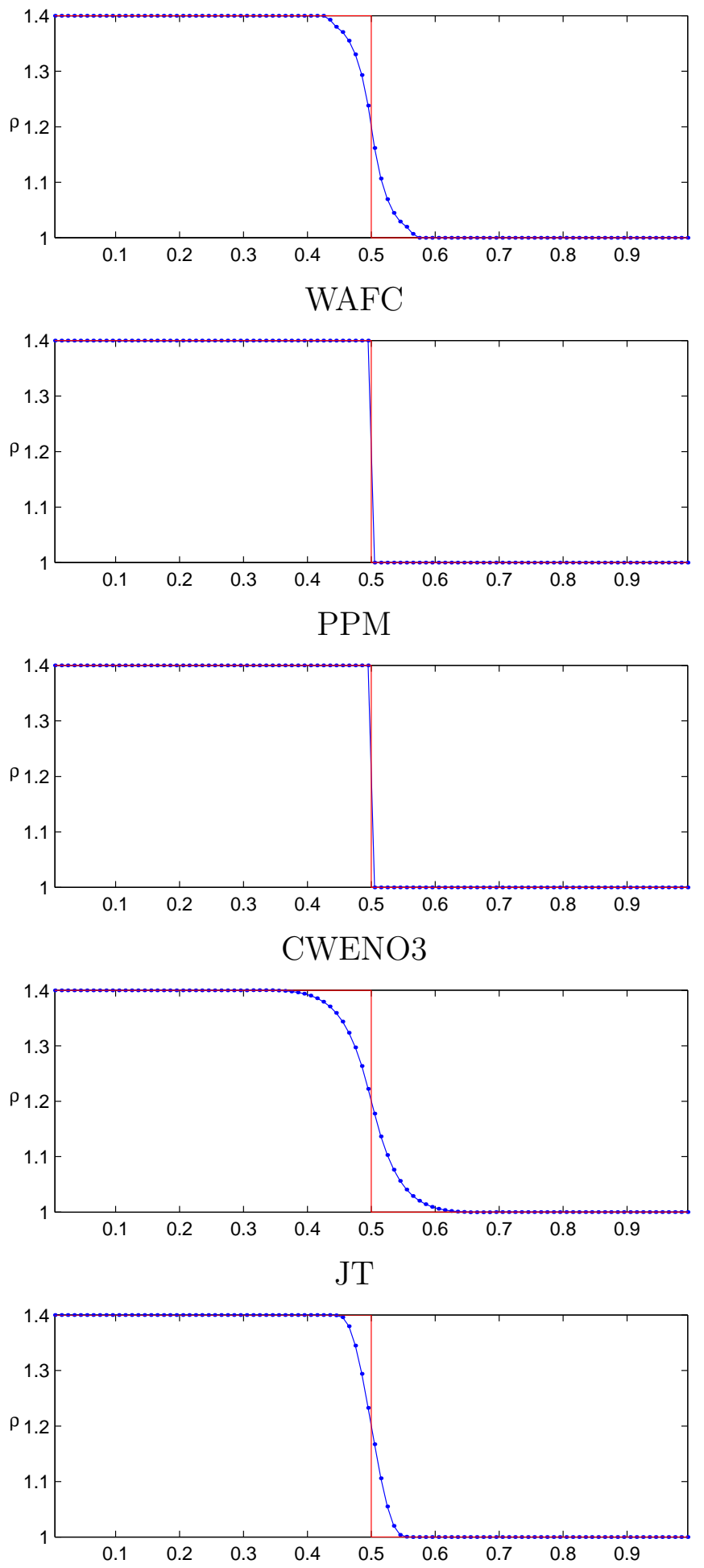

Figure 7: 1D results (density) for the test 5 problem by all ten schemes. 


\section{Test 6}

\section{CFLF}
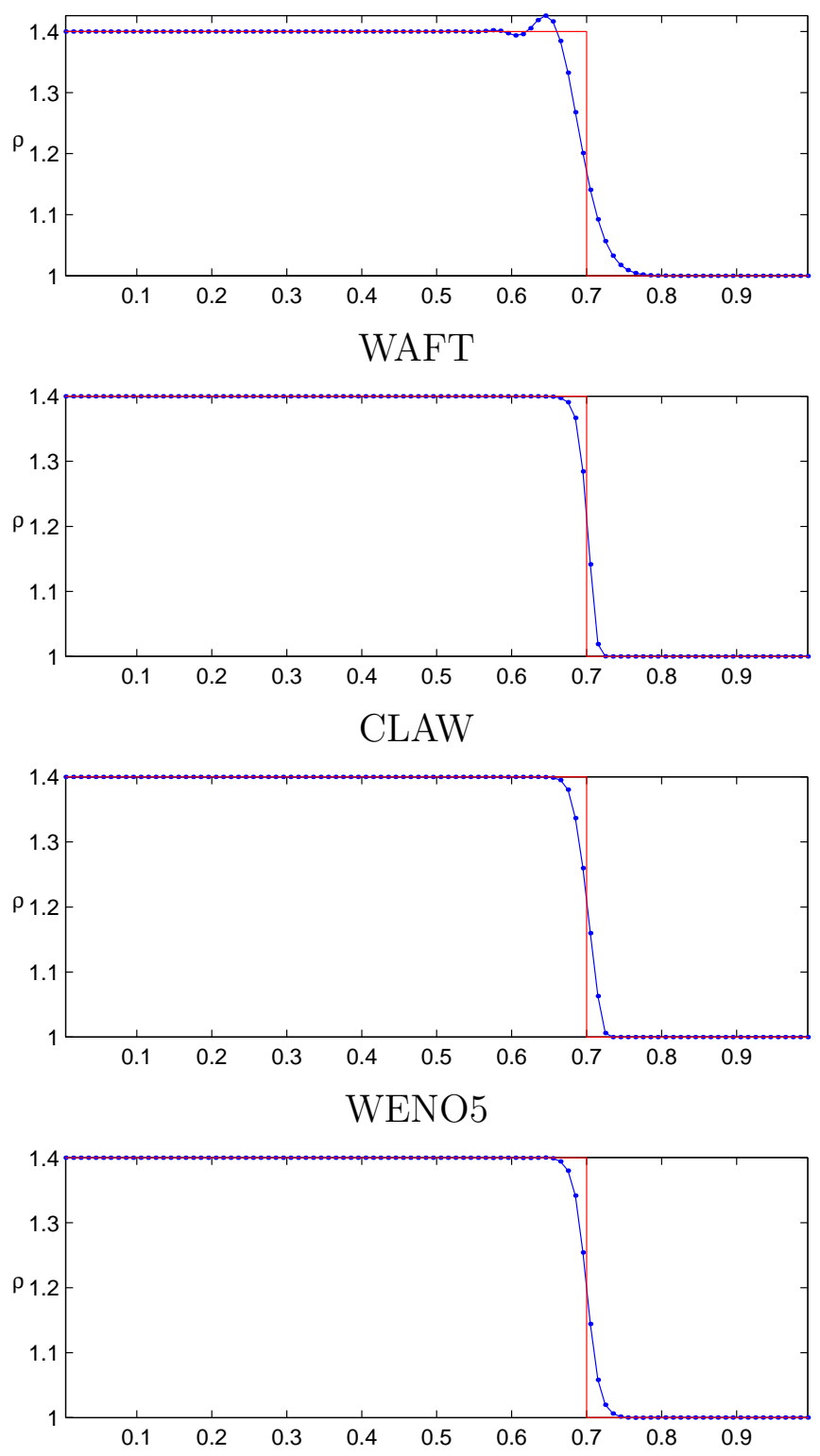

LL

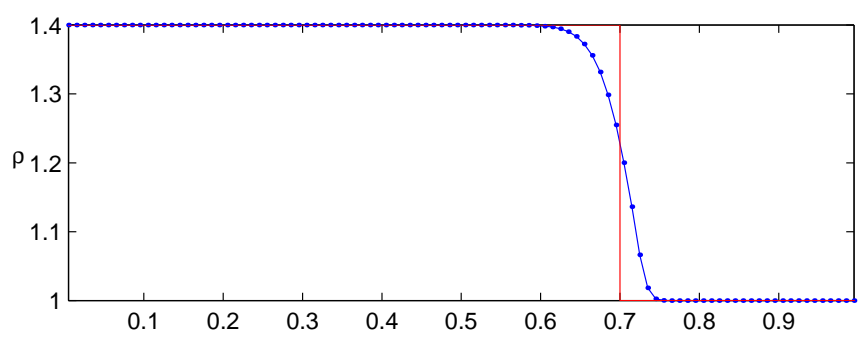

CFLFh
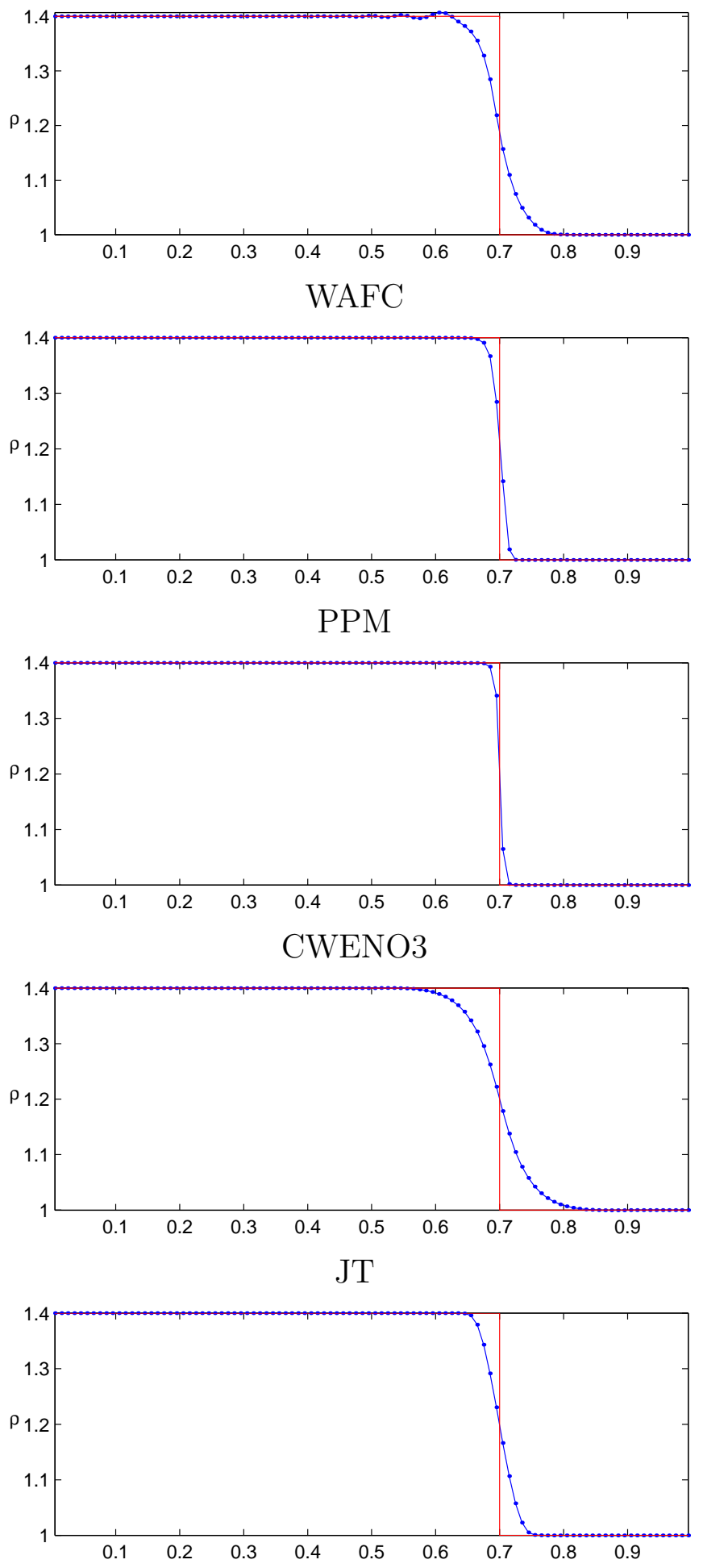

Figure 8: 1D results (density) for the test 6 problem by all ten schemes. 


\section{Peak}

CFLF
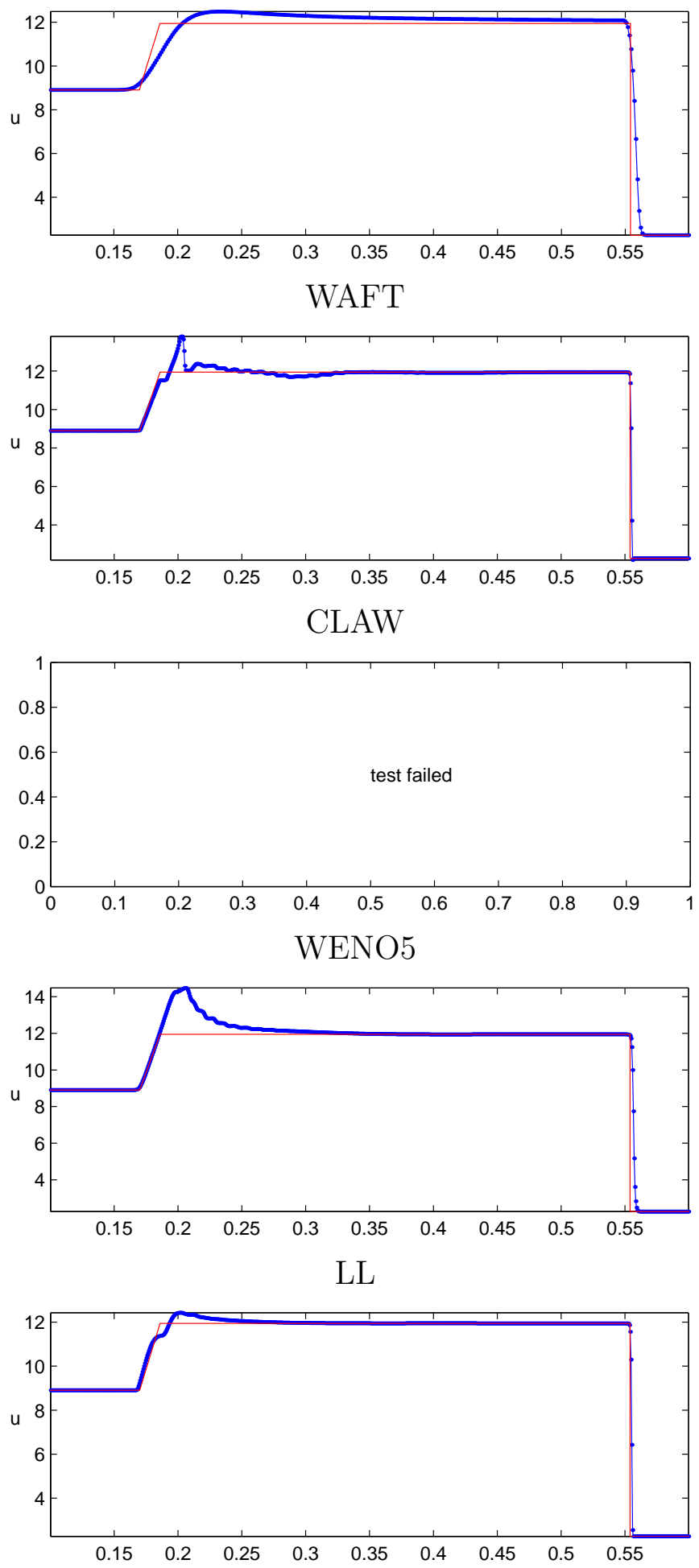

CFLFh
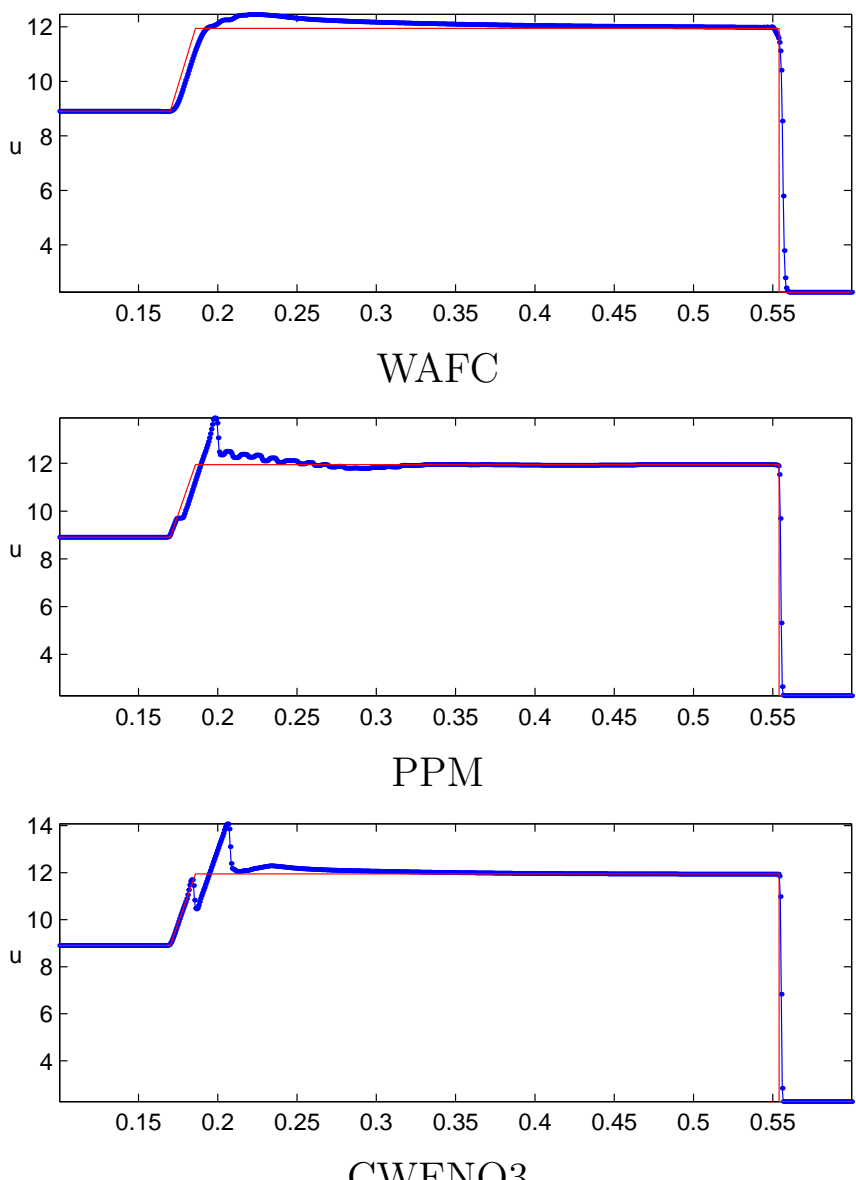

CWENO3
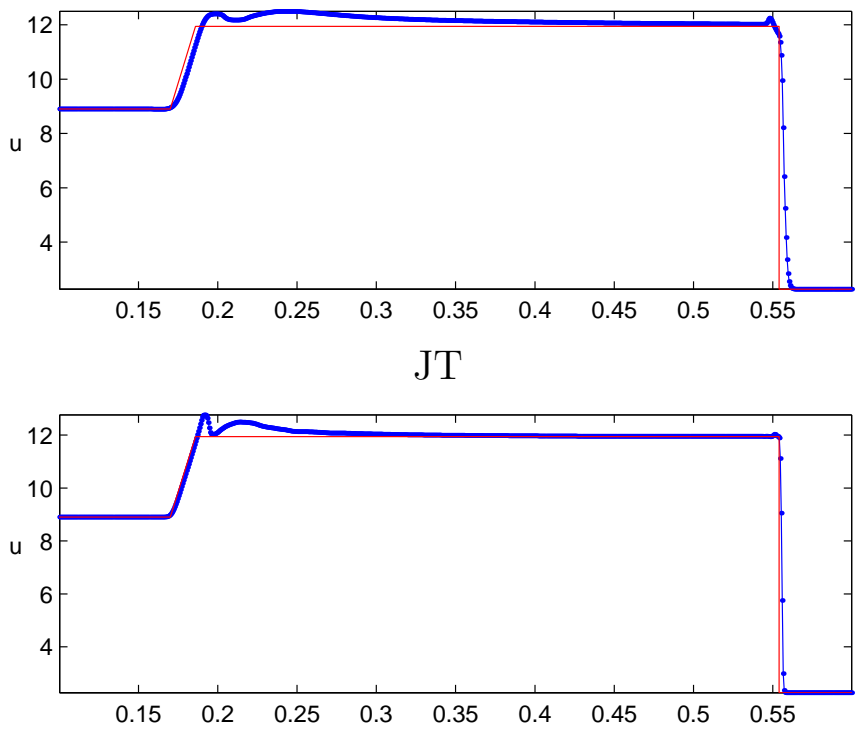

Figure 9: 1D results (velocity) for the peak problem by all ten schemes. 


\section{Peak density}

\section{CFLF}
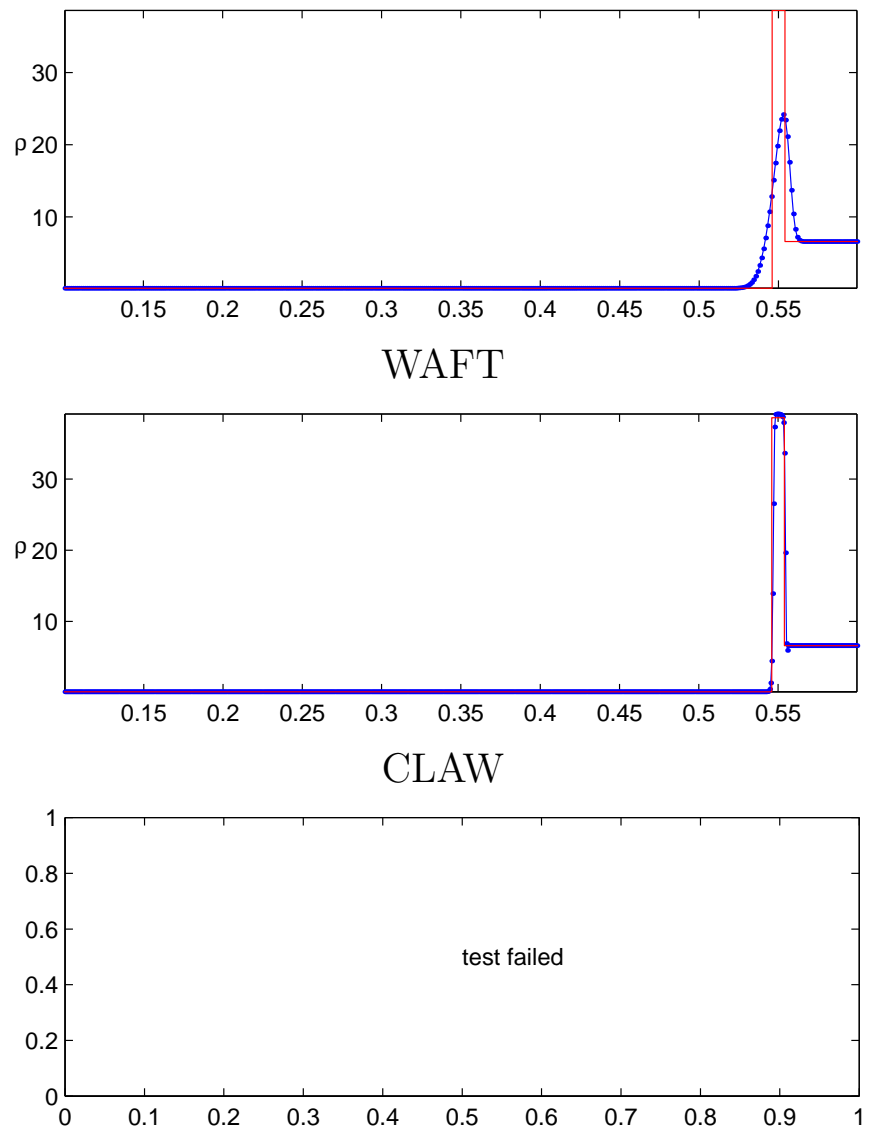

WENO5

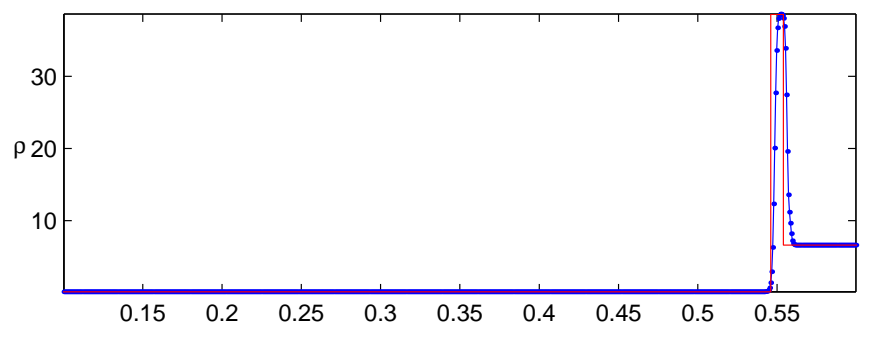

LL

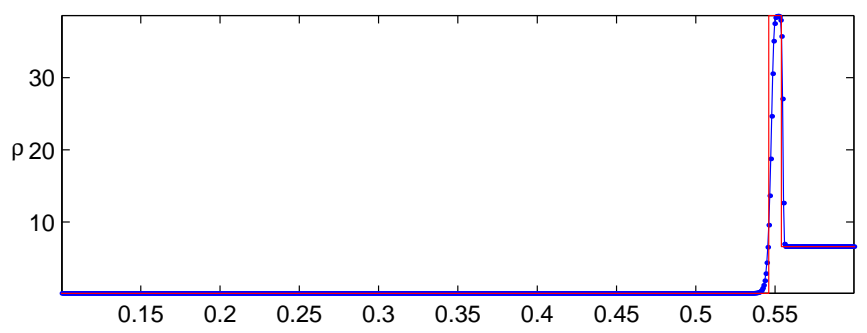

CFLFh
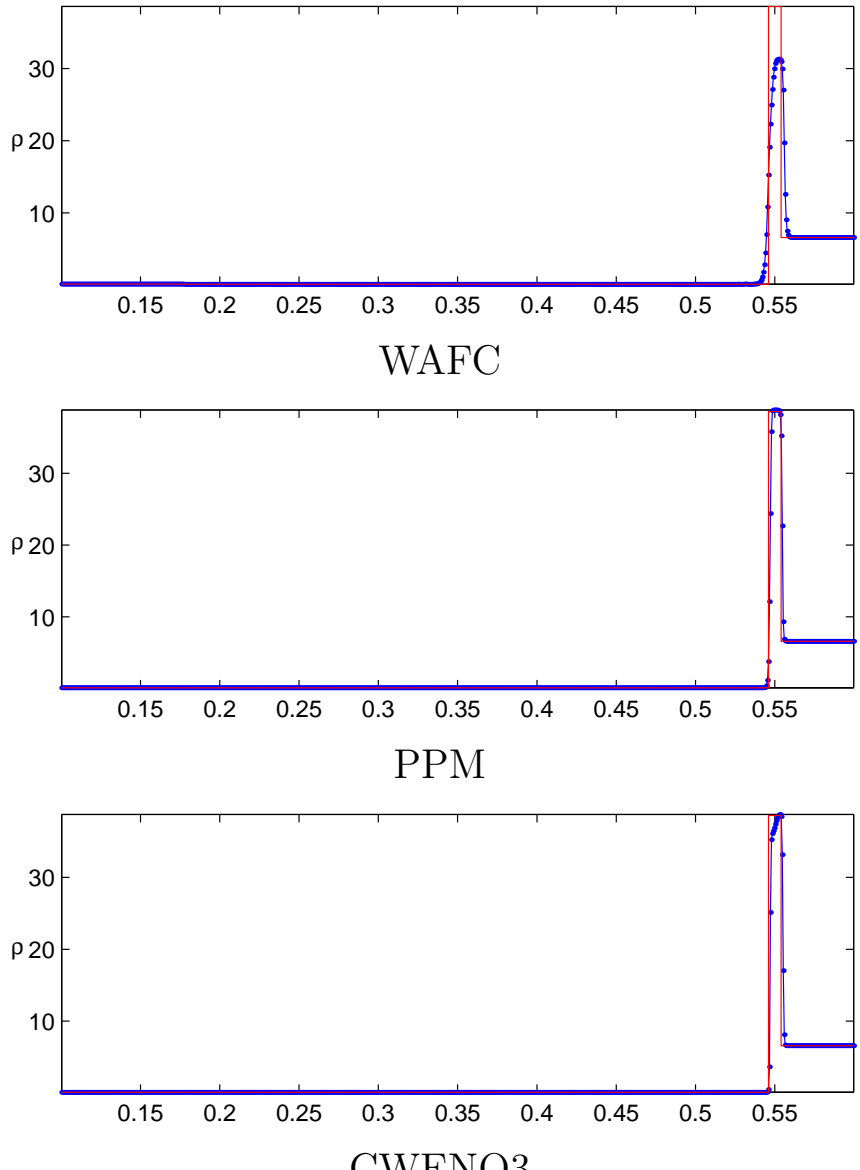

CWENO3
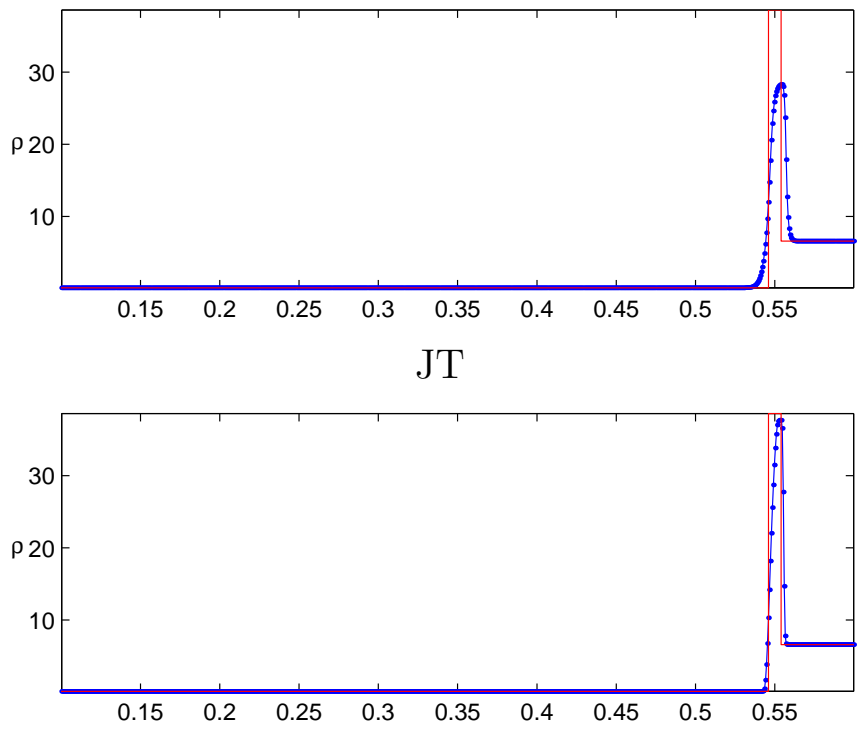

Figure 10: 1D results (density) for the peak problem by all ten schemes. 


\section{Blast wave on 400 cells}

CFLF
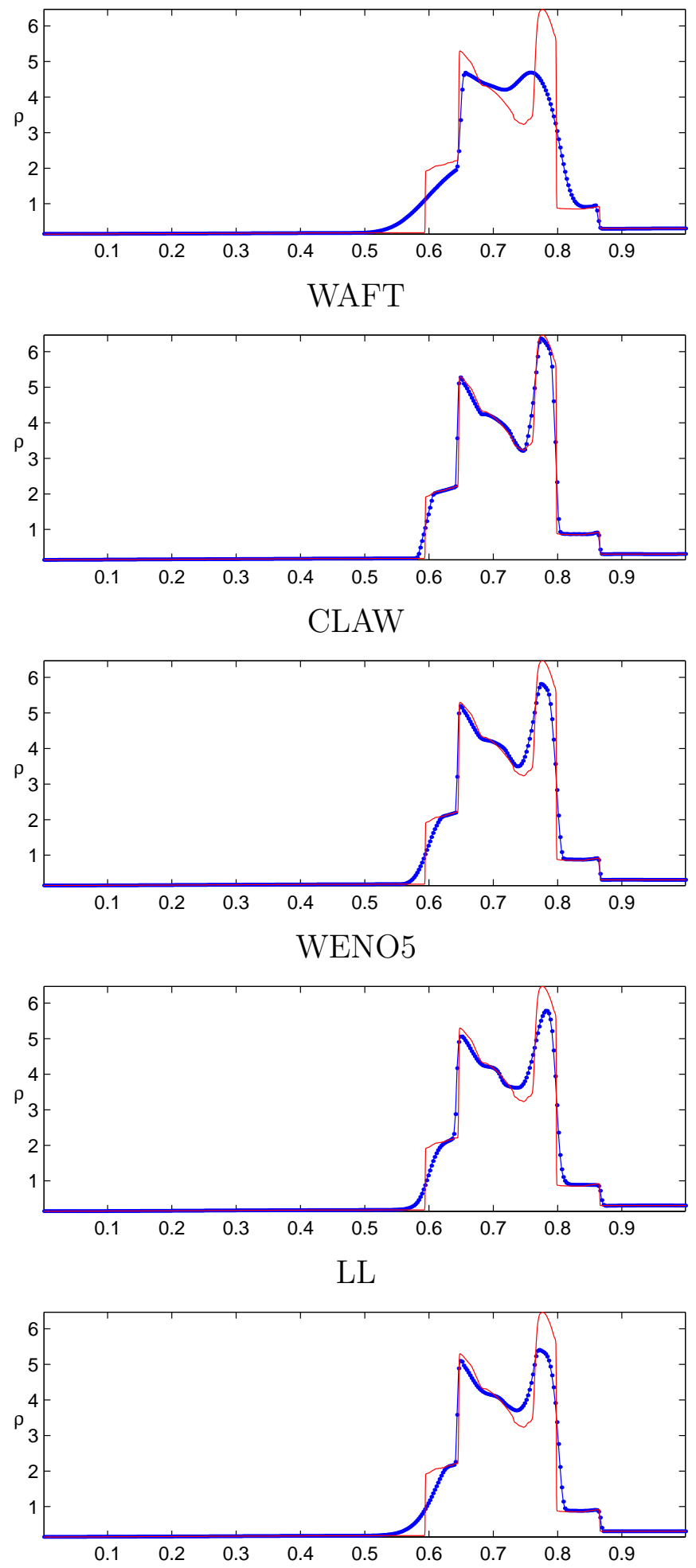

CFLFh
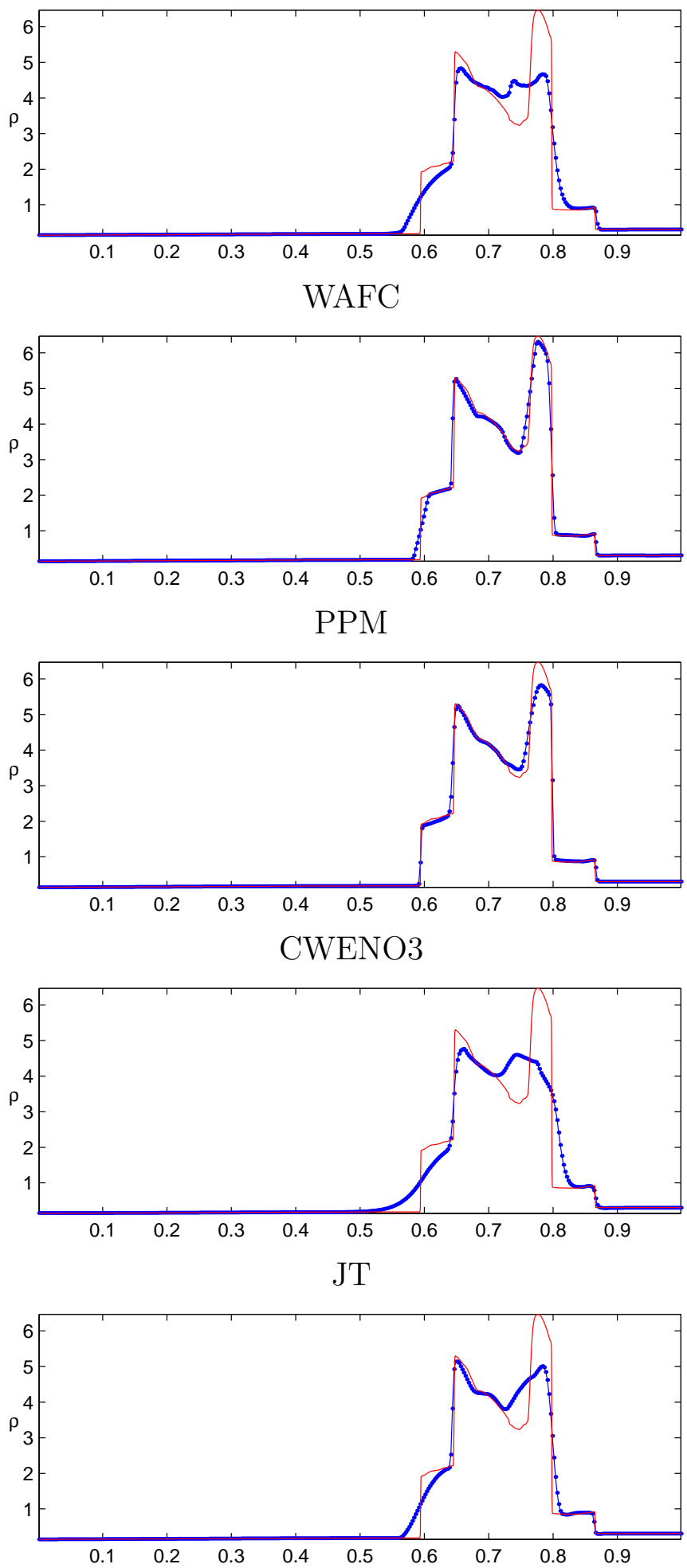

Figure 11: 1D results (density) for the blast wave problem by all ten schemes. 


\section{Blast wave on 2000 cells}

CFLF
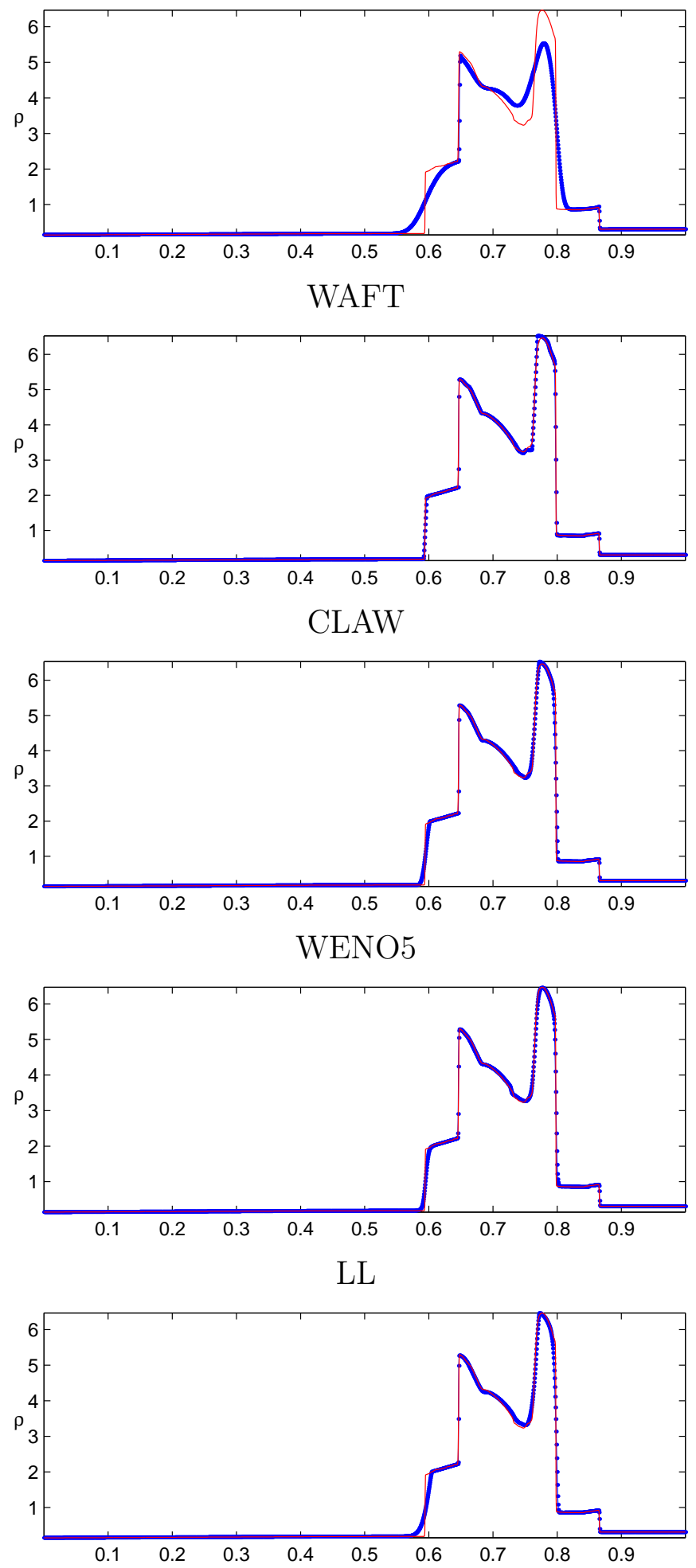

CFLFh
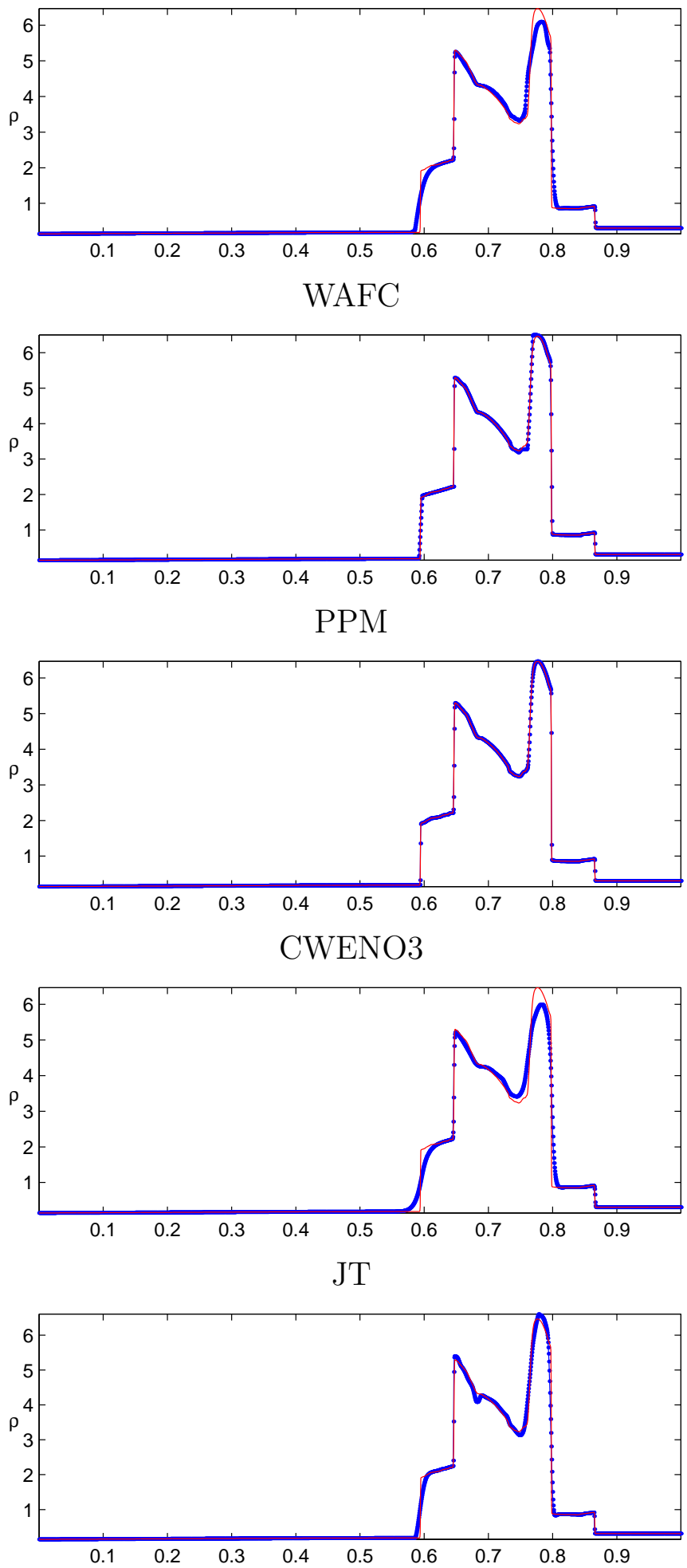

Figure 12: 1D results (density) for the blast wave problem by all ten schemes. 


\section{$42 \mathrm{D}$ tests}

\subsection{Accuracy - Smooth periodic problem}

To check the accuracy of the presented schemes we have computed the numerical solution of an exact smooth solution [33]

$$
\rho(x, y, t)=1+0.2 \sin (\pi(x+y-t(u+v))), \quad u, v, p \text { constants }
$$

of the Euler equations for an ideal gas. We have used the particular values $u=1, v=-1 / 2, p=1$ for velocities and pressure. The gas constant is again $\gamma=1.4$. Periodic boundary conditions are

\begin{tabular}{|c|c|c|c|c|c|c|c|}
\hline Test Scheme & 25 & Order & 50 & Order & 100 & Order & 200 \\
\hline & \multicolumn{7}{|c|}{ relative $L^{1}$ errors } \\
\hline CFLF & $7.1 \mathrm{e}+00$ & 0.7 & $4.2 \mathrm{e}+00$ & 0.9 & $2.3 e+00$ & $\overline{0.9}$ & $1.2 \mathrm{e}+00$ \\
\hline CFLFh & $2.5 \mathrm{e}+00$ & 2.3 & $5.1 \mathrm{e}-01$ & 2.1 & $1.2 \mathrm{e}-01$ & 2.0 & $3.1 \mathrm{e}-02$ \\
\hline WAFT & $5.7 \mathrm{e}-01$ & -0.1 & $6.0 \mathrm{e}-01$ & 1.4 & $2.3 \mathrm{e}-01$ & 1.8 & $6.7 \mathrm{e}-02$ \\
\hline WAF4 & $5.7 \mathrm{e}-01$ & 0.3 & $4.7 \mathrm{e}-01$ & 1.1 & $2.2 \mathrm{e}-01$ & 1.8 & $6.5 \mathrm{e}-02$ \\
\hline CLAW & $4.1 \mathrm{e}-01$ & 2.5 & $7.3 \mathrm{e}-02$ & 2.2 & $1.6 \mathrm{e}-02$ & 2.2 & $3.7 \mathrm{e}-03$ \\
\hline PPM & $2.4 \mathrm{e}-02$ & 3.1 & $2.8 \mathrm{e}-03$ & 3.0 & $3.4 \mathrm{e}-04$ & 3.0 & $4.3 \mathrm{e}-05$ \\
\hline WENO & $3.1 \mathrm{e}-02$ & 5.0 & $9.7 \mathrm{e}-04$ & 5.0 & $3.1 \mathrm{e}-05$ & 4.5 & $1.3 \mathrm{e}-06$ \\
\hline CWENO & $7.3 e+00$ & 1.9 & $1.9 \mathrm{e}+00$ & 1.8 & $5.6 \mathrm{e}-01$ & 3.0 & $7.2 \mathrm{e}-02$ \\
\hline $\mathrm{LL}$ & $2.0 \mathrm{e}+00$ & 1.3 & 8.0e-01 & 1.9 & $2.1 \mathrm{e}-01$ & 2.0 & $5.4 \mathrm{e}-02$ \\
\hline \multirow[t]{2}{*}{ JT } & $1.1 \mathrm{e}+00$ & 2.3 & $2.3 \mathrm{e}-01$ & 1.9 & $5.9 \mathrm{e}-02$ & 1.9 & $1.6 \mathrm{e}-02$ \\
\hline & \multicolumn{7}{|c|}{ relative $L^{2}$ errors } \\
\hline CFLF & $7.8 \mathrm{e}+00$ & 0.7 & $4.7 \mathrm{e}+00$ & 0.9 & $2.6 \mathrm{e}+00$ & $\overline{0.9}$ & $1.3 \mathrm{e}+00$ \\
\hline CFLFh & $2.7 \mathrm{e}+00$ & 2.2 & $5.7 \mathrm{e}-01$ & 2.1 & $1.3 \mathrm{e}-01$ & 2.0 & $3.4 \mathrm{e}-02$ \\
\hline WAFT & $6.5 \mathrm{e}-01$ & -0.0 & $6.7 \mathrm{e}-01$ & 1.3 & $2.8 \mathrm{e}-01$ & 1.6 & $9.1 \mathrm{e}-02$ \\
\hline WAF4 & $7.4 \mathrm{e}-01$ & 0.4 & $5.6 \mathrm{e}-01$ & 1.0 & $2.7 \mathrm{e}-01$ & 1.6 & $8.8 \mathrm{e}-02$ \\
\hline CLAW & $5.6 \mathrm{e}-01$ & 2.3 & $1.1 \mathrm{e}-01$ & 2.1 & $2.6 \mathrm{e}-02$ & 2.1 & $6.0 \mathrm{e}-03$ \\
\hline PPM & $2.7 \mathrm{e}-02$ & 3.1 & $3.1 \mathrm{e}-03$ & 3.0 & $3.8 \mathrm{e}-04$ & 3.0 & $4.7 \mathrm{e}-05$ \\
\hline WENO & $3.4 \mathrm{e}-02$ & 5.0 & $1.1 \mathrm{e}-03$ & 5.0 & $3.4 \mathrm{e}-05$ & 4.5 & $1.5 \mathrm{e}-06$ \\
\hline CWENO & $8.3 e+00$ & 1.8 & $2.4 \mathrm{e}+00$ & 1.8 & $6.9 \mathrm{e}-01$ & 2.7 & $1.1 \mathrm{e}-01$ \\
\hline LL & $2.6 \mathrm{e}+00$ & 1.4 & $9.5 \mathrm{e}-01$ & 1.7 & $2.9 \mathrm{e}-01$ & 1.7 & $8.8 \mathrm{e}-02$ \\
\hline \multirow[t]{2}{*}{ JT } & $1.2 \mathrm{e}+00$ & 2.2 & $2.5 \mathrm{e}-01$ & 2.0 & $6.3 \mathrm{e}-02$ & 1.9 & $1.7 \mathrm{e}-02$ \\
\hline & \multicolumn{7}{|c|}{ relative $\max$ errors } \\
\hline CFLF & $9.3 e+00$ & 0.8 & $5.6 \mathrm{e}+00$ & 0.9 & $3.1 \mathrm{e}+00$ & 0.9 & $1.6 \mathrm{e}+00$ \\
\hline CFLFh & $3.5 \mathrm{e}+00$ & 2.1 & $8.2 \mathrm{e}-01$ & 2.3 & $1.7 \mathrm{e}-01$ & 2.1 & $4.0 \mathrm{e}-02$ \\
\hline WAFT & $1.0 \mathrm{e}+00$ & -0.1 & $1.1 \mathrm{e}+00$ & 0.9 & $5.7 \mathrm{e}-01$ & 1.2 & $2.5 \mathrm{e}-01$ \\
\hline WAF4 & $1.1 \mathrm{e}+00$ & 0.2 & $1.0 \mathrm{e}+00$ & 0.8 & $6.0 \mathrm{e}-01$ & 0.8 & $3.5 \mathrm{e}-01$ \\
\hline CLAW & $1.1 \mathrm{e}+00$ & 2.0 & $2.8 \mathrm{e}-01$ & 1.6 & $9.0 \mathrm{e}-02$ & 1.7 & $2.7 \mathrm{e}-02$ \\
\hline PPM & $3.2 \mathrm{e}-02$ & 3.1 & $3.7 \mathrm{e}-03$ & 3.0 & $4.5 \mathrm{e}-04$ & 3.0 & $5.6 \mathrm{e}-05$ \\
\hline WENO & $4.0 \mathrm{e}-02$ & 4.8 & $1.4 \mathrm{e}-03$ & 4.9 & $4.7 \mathrm{e}-05$ & 4.0 & $2.9 \mathrm{e}-06$ \\
\hline CWENO & $1.1 \mathrm{e}+01$ & 1.5 & $3.7 \mathrm{e}+00$ & 1.7 & $1.2 \mathrm{e}+00$ & 2.2 & $2.5 \mathrm{e}-01$ \\
\hline LL & $4.1 \mathrm{e}+00$ & 1.3 & $1.6 \mathrm{e}+00$ & 1.3 & $6.6 \mathrm{e}-01$ & 1.3 & $2.6 \mathrm{e}-01$ \\
\hline JT & $1.4 \mathrm{e}+00$ & 2.2 & $3.0 \mathrm{e}-01$ & 2.0 & $7.4 \mathrm{e}-02$ & 1.6 & $2.5 \mathrm{e}-02$ \\
\hline
\end{tabular}

Table 3: Relative $L^{1}, L^{2}$ and $\max$ density errors in $\%$ for $2 \mathrm{D}$ smooth periodic problem for all ten schemes on refined grid with orders of accuracy. 
employed. The problem is run on the series of refined grids with $25 \times 25,50 \times 50,100 \times 100,200 \times 200$ cells until the final time $T=4$ giving the movement of the wave by one full period. Results for all schemes are summarized in Table 3 for relative max, $L^{1}$ and $L^{2}$ norms density errors which are shown in \%. The order of accuracy of schemes in this table is computed as the base 2 logarithm of the ratio of two errors from neighboring columns.

From the table we see that the most accurate is 5-th order WENO5 followed by 3-rd order PPM. Also both these schemes keep the high accuracy from very rough grids for all three types of errors. Most schemes are second order and CFLF is just first order.

\subsection{Speed}

To compare the schemes regarding their speed we have measured CPU time (on an SGI Origin with 250 Mhz MIPS R10000 processor - we have PPM available only on SGI machines) for all of them for 2D Riemann problem Case 4 described in the section 4.3 on the grid of $400 \times 400$ cells up to time $T=0.05$. The results are summarized in Table 4 showing also the ratio how many times the given scheme is slower than the fastest scheme from our ten schemes (WAFC) and number of adaptive time steps used by the different schemes. From the number of steps one can note that last four schemes (WENO5, CWENO3, LL, JT) need about twice as much time as the first six schemes. In fact, the first six schemes use a CFL limit of one, while the last four need CFL number $1 / 2$. Of course this means that the last four schemes using $C F L=1 / 2$ are slow except JT which is remarkably fast with $C F L=1 / 2$. WENO5 is the slowest using $C F L=1 / 2$ and eigenvector decomposition. WENO5, CWENO3 and LL are slow, all others are quite fast.

\begin{tabular}{|l|rrr|}
\hline scheme & CPU time[s] & ratio & time steps \\
\hline CFLF & 95 & 1.0 & 47 \\
CFLFh & 133 & 1.4 & 46 \\
WAFT & 150 & 1.6 & 46 \\
WAFC & 93 & 1.0 & 48 \\
CLAW & 118 & 1.3 & 49 \\
PPM & 140 & 1.5 & 46 \\
WENO5 & 570 & 6.1 & 89 \\
CWENO3 & 271 & 2.9 & 87 \\
LL & 310 & 3.3 & 88 \\
JT & 103 & 1.1 & 92 \\
\hline
\end{tabular}

Table 4: Exacution CPU times for 2D Riemann problem Case 4 on the grid of $400 \times 400$ cells up to time $T=0.05$; ratio between the CPU time of given scheme and that one of the fastest scheme (WAFC); number of time steps used by each scheme.

\subsection{Description of 2D Riemann problems}

We have taken six cases from the collection of 2D Riemann problems proposed by [43] and used by others [23, 44], namely, cases 3,4,6,12,15 and 17 from [23] (which are configurations 3, 4, B, $F, G, K$ from [43] $)$. These problems are solved on the square $(x, y) \in(0,1) \times(0,1)$. The square is divided into four quadrants by lines $x=1 / 2, y=1 / 2$. The Riemann problems are defined by initial constant states in each quadrant. These initial states in left/right-upper/lower quadrants 
for the pressure $p$, density $\rho, x$-component of velocity $u$ and $y$-component of the velocity $v$ are presented at Table 5 together with the time $T$ at which the results are presented. All these problems use the gas constant $\gamma=1.4$. In the figures presenting results we use the same set of contours for density as has been used in [43]. All the Riemann problems in [43] are proposed in such a way that the solutions of all four 1D Riemann problems between quadrants have exactly one wave (shock, rarefaction or contact-slip). Following [2:3], let $R$ stand for rarefaction, $S$ for shock, and $J$ for contact-slip. Starting at the left side and going clockwise, the cases are: Case 3: $S, S, S, S$, Case 4: $S, S, S, S$, Case 6: $J, J, J, J$, Case 12: J, S, S, J, Case 15: $J, R, S, J$, Case 17: $S, J, R, J$.

\begin{tabular}{|c|l|llll|llll|l|}
\hline Case & & \multicolumn{5}{|c|}{ left } & \multicolumn{5}{c|}{ right } & T \\
& & $p$ & $\rho$ & $u$ & $v$ & $p$ & $\rho$ & $u$ & $v$ & \\
\hline \multirow{3}{*}{3} & upper & 0.3 & 0.5323 & 1.206 & 0.0 & 1.5 & 1.5 & 0.0 & 0.0 & \\
& lower & 0.029 & 0.138 & 1.206 & 1.206 & 0.3 & 0.5323 & 0.0 & 1.206 & 0.3 \\
4 & upper & 0.35 & 0.5065 & 0.8939 & 0.0 & 1.1 & 1.1 & 0.0 & 0.0 & \\
& lower & 1.1 & 1.1 & 0.8939 & 0.8939 & 0.35 & 0.5065 & 0.0 & 0.8939 & 0.25 \\
\hline \multirow{2}{*}{6} & upper & 1.0 & 2.0 & 0.75 & 0.5 & 1.0 & 1.0 & 0.75 & -0.5 & \\
& lower & 1.0 & 1.0 & -0.75 & 0.5 & 1.0 & 3.0 & -0.75 & -0.5 & 0.3 \\
\hline \multirow{3}{*}{12} & upper & 1.0 & 1.0 & 0.7276 & 0.0 & 0.4 & 0.5313 & 0.0 & 0.0 & \\
& lower & 1.0 & 0.8 & 0.0 & 0.0 & 1.0 & 1.0 & 0.0 & 0.7276 & 0.25 \\
\hline \multirow{3}{*}{15} & upper & 0.4 & 0.5197 & -0.6259 & -0.3 & 1.0 & 1.0 & 0.1 & -0.3 & \\
17 & lower & 0.4 & 0.8 & 0.1 & -0.3 & 0.4 & 0.5313 & 0.1 & 0.4276 & 0.2 \\
\hline \multirow{2}{*}{17} & upper & 1.0 & 2.0 & 0.0 & -0.3 & 1.0 & 1.0 & 0.0 & -0.4 & \\
\end{tabular}

Table 5: Initial states in four left/right-upper/lower quadrants for 2D Riemann problems for the pressure $p$, density $\rho, x$-component of velocity $u$ and $y$-component of the velocity $v . T$ is the final time. 


\subsection{Results for 2D Riemann problems}

As we stated in the introduction, exact solutions are not known for these two-dimensional Riemann

problems. Furthermore, the color maps can hide details such as small oscillations. But by having pressure as color and density as contours, it is at least possible to see what the schemes think the structures are. In some cases there are clear errors caused by a poor resolution of the initial 1D problems.

Results are shown in the following figures. These runs are for grids with $400 \times 400$ cells. Color pressure map is overlayed by density contours and velocity arrows.

Case 3: WAFC is noisier than the others. One can notice the different resolution (by different schemes) of the four 1D shocks separating the four regions of constant states. To some extent all schemes agree on the basic structure of the solution in the region where these four shocks interact.

Note the artefacts remaining for some schemes on two segments of the initial discontinuities between the upper right quadrant and upper left and lower right quadrants. When we look at these more detail we find that these errors are present for all schemes, it is just that for some schemes (CFLFh, WAFT, WAFC, CLAW and PPM) they are large enough so that they are visible in the choosen density contours. Even further, when we try to compute just the 1D Riemann problem defined between the two upper quadrants (or equivalently between the two right quadrants), these artefacts (dip in density) are present for all schemes in the 1D results. Four schemes (CFLFh, WAFT, WAFC and PPM) show also such errors at the other two initial inner jump segments between the lower left quadrant and two of its neighbors on the right and at the top. This is sometimes the price paid for good contact resolution, there is not enough dissipation to reduce a residual error in density. 


\section{Case 3}

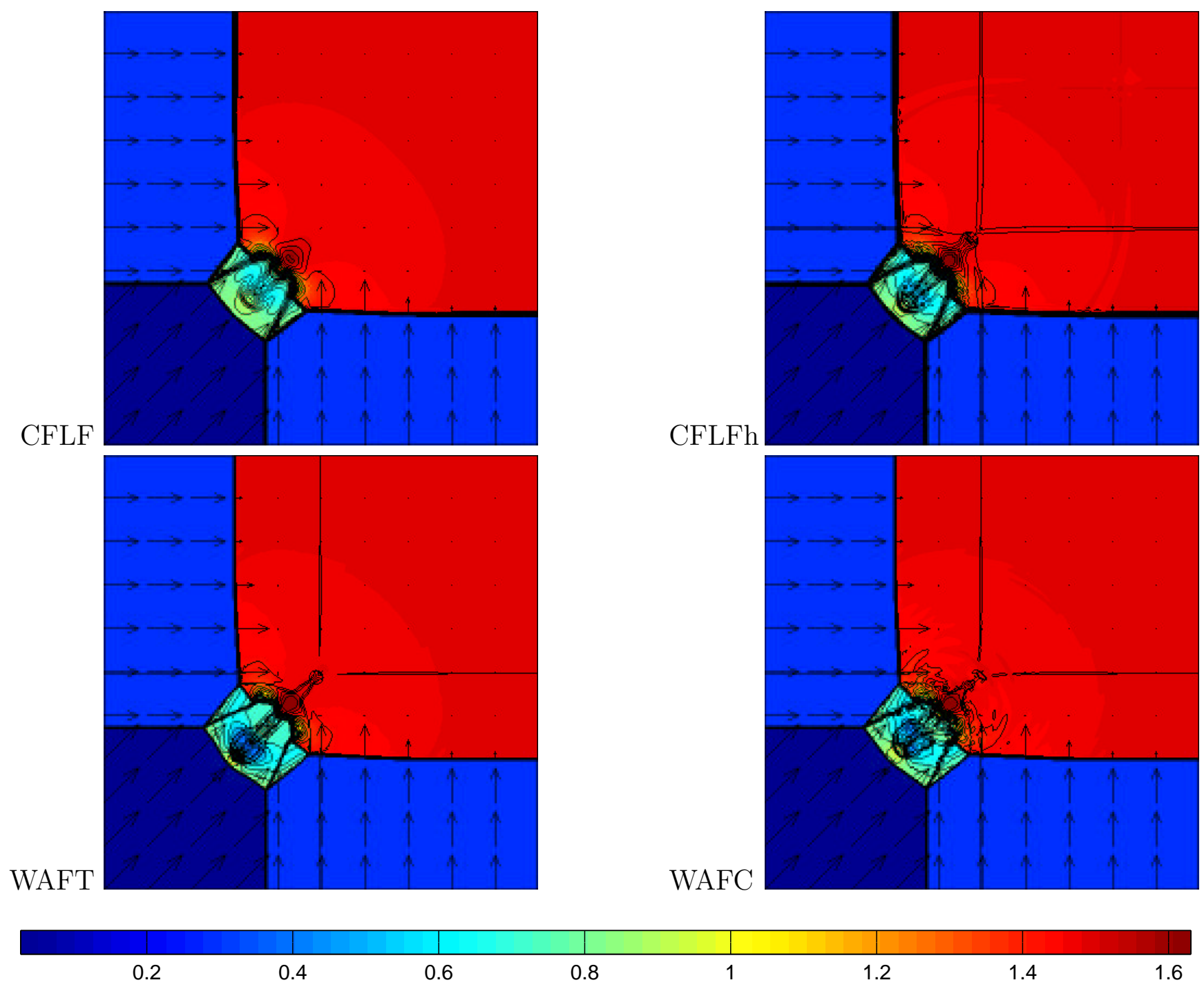

Figure 13: Results for the 2D Riemann problem case 3 by four schemes with pressure colorbar. Pressure is displayed by color, density by 32 contours ( 0.16 to 1.71 step 0.05$)$ and velocity by arrows. For all cases the computations were done and are presented on the square $(x, y) \in$ $(0,1) \times(0,1)$. 


\section{Case 3}
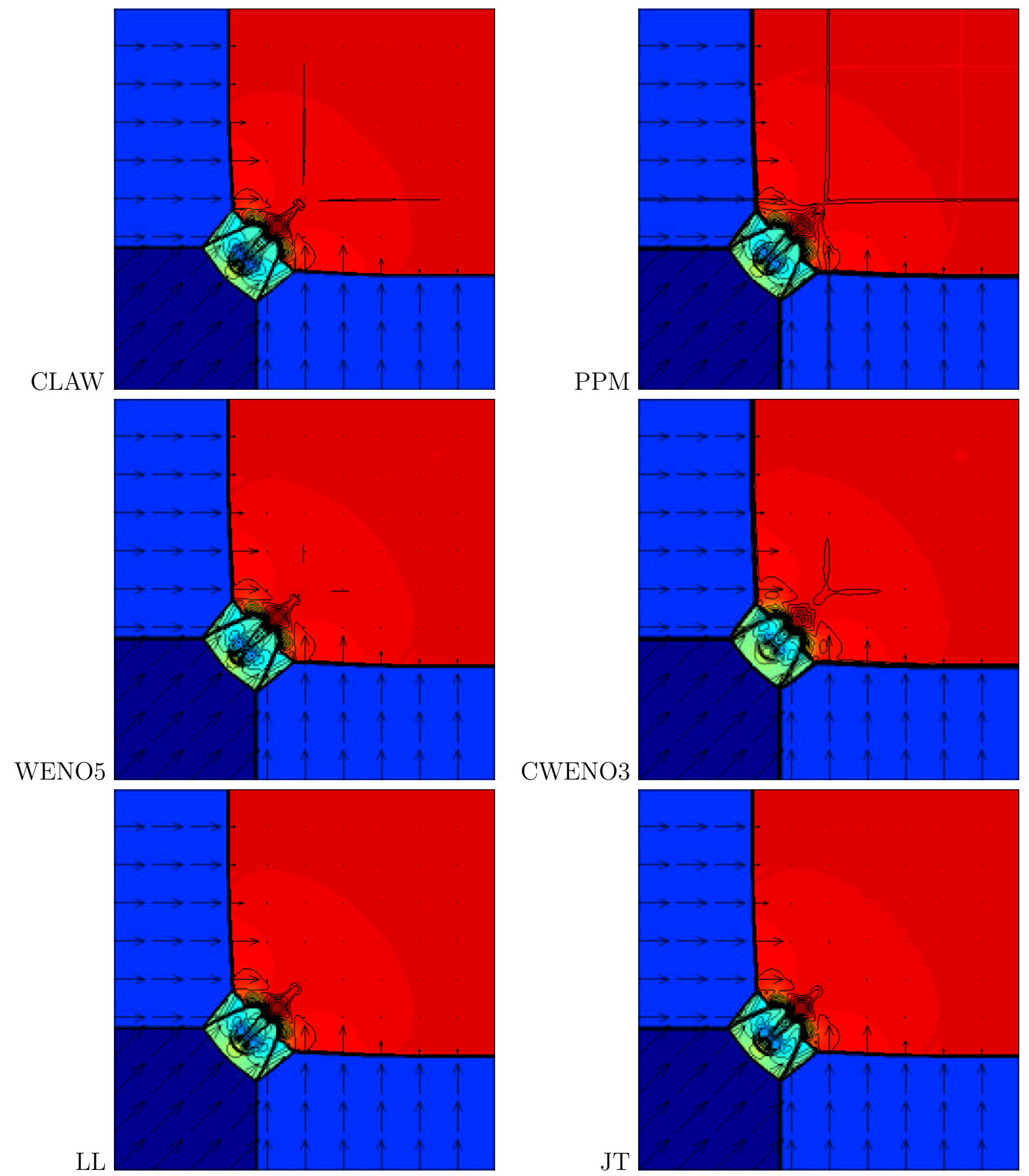

Figure 14: Results for the 2D Riemann problem case 3 by remaining six schemes. Pressure is displayed by color, density by 32 contours (0.16 to 1.71 step 0.05$)$ and velocity by arrows. For all cases the computations were done and are presented on the square $(x, y) \in(0,1) \times(0,1)$. 
Case 4: CFLFh is noisy, WAFC somewhat less so. The other methods are very similar to each other. Most methods resolve well all the shocks, both the straight 1D shocks separating two constant states and the two curved shocks bordering the lens shaped region of higher density and pressure. The solution in this region should be symmetric about the lens axis (if we woud stay in a coordinate system fixed to this axis, which is moving with constant speed, the problem would be symmetric about this axis). Some schemes do not keep this symmetry in all details, e.g. CFLF and JT have differences along the upper and lower curved shocks. WAFC has an artefact in density similar to those in the previous case 3 .

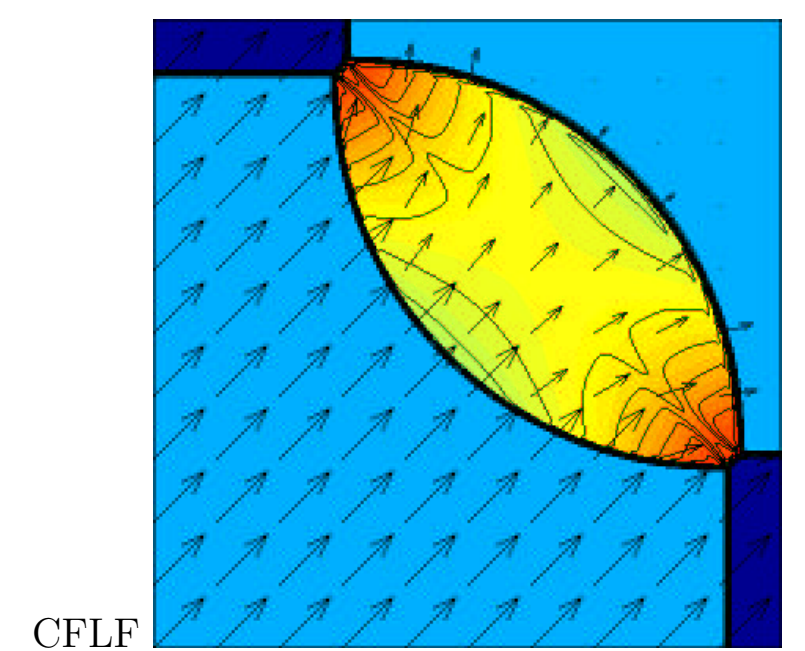

WAFT

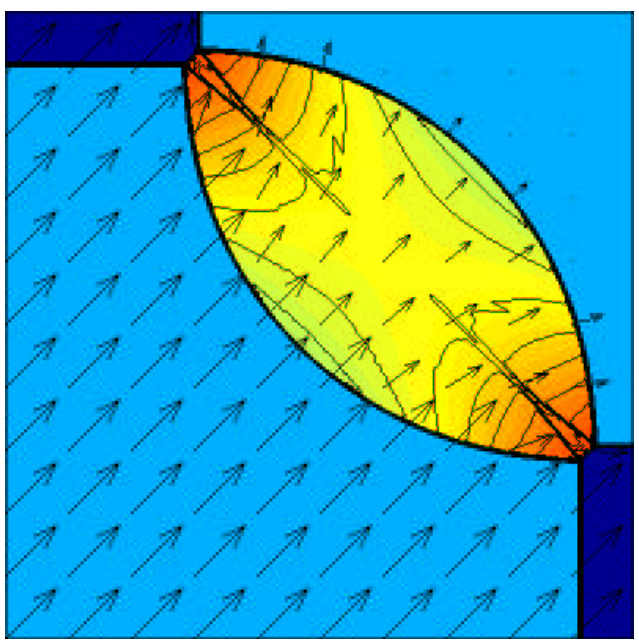

CFLFh

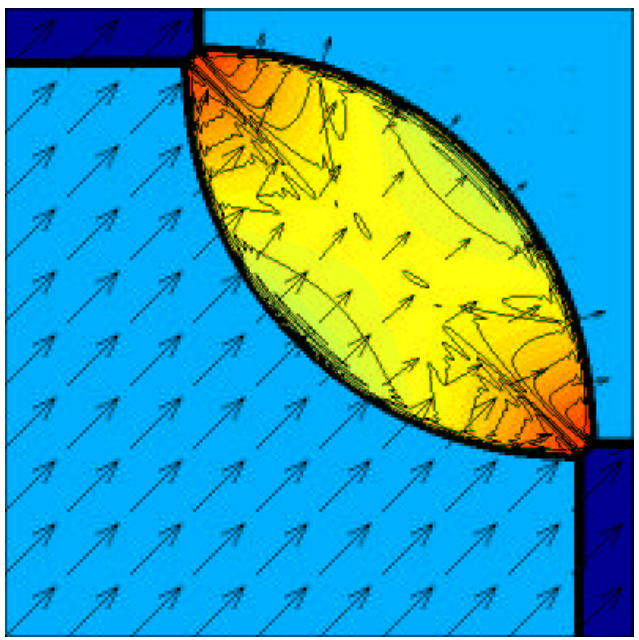

WAFC

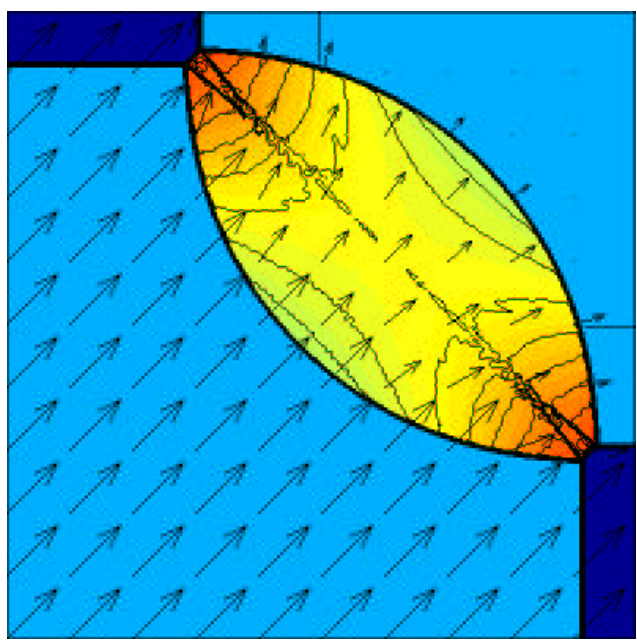

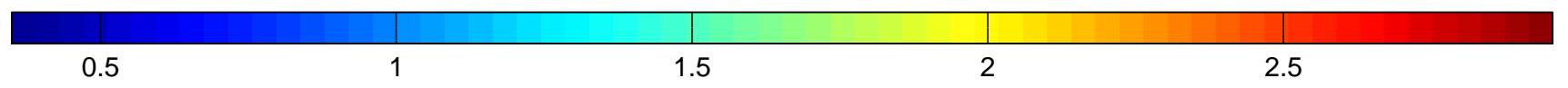

Figure 15: Results for the 2D Riemann problem case 4 by four schemes with pressure colorbar. Pressure is displayed by color, density by 29 contours (0.52 to 1.92 step 0.05 ) and velocity by arrows. For all cases the computations were done and are presented on the square $(x, y) \in$ $(0,1) \times(0,1)$ 


\section{Case 4}
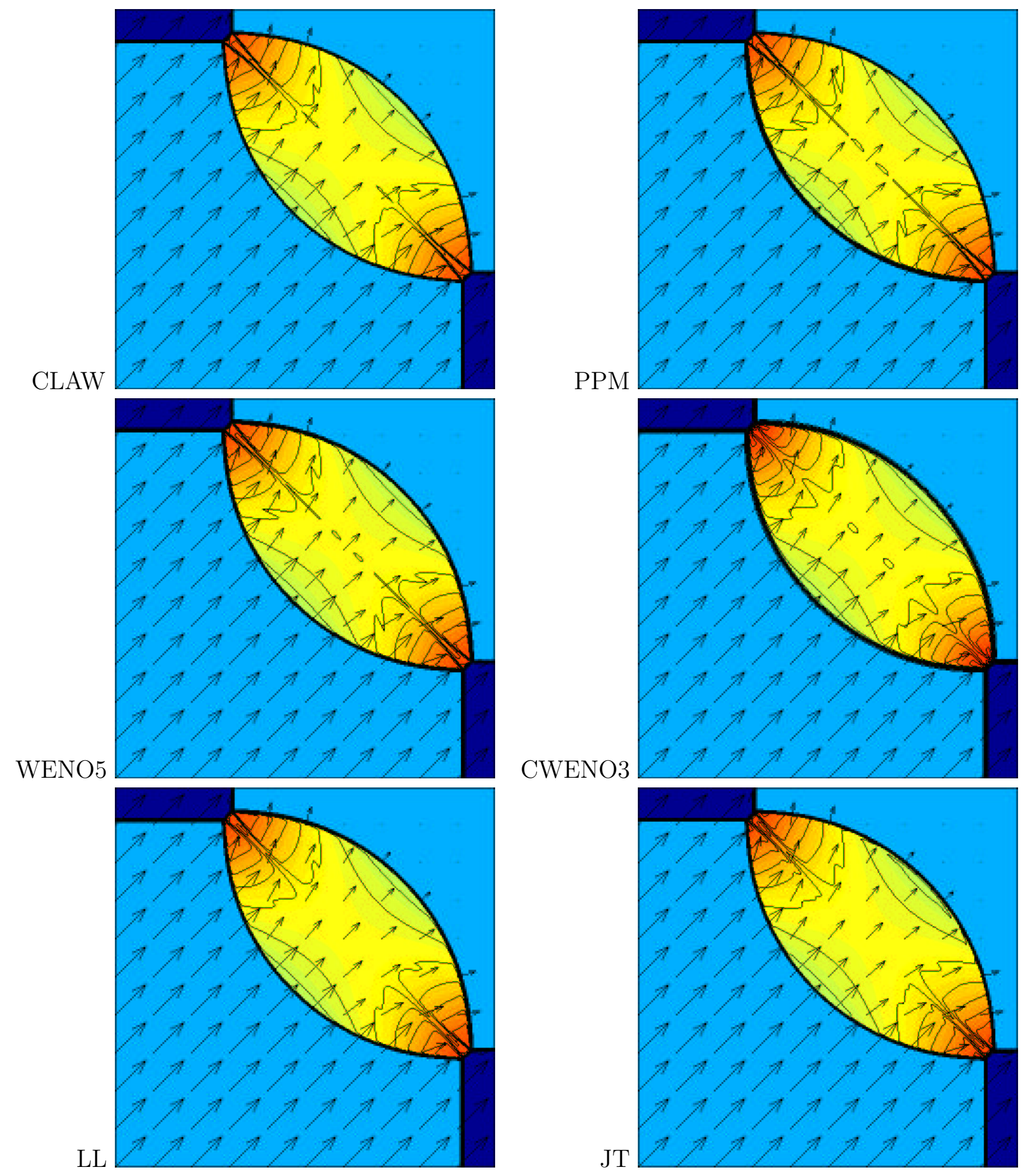

Figure 16: Results for the 2D Riemann problem case 4 by remaining six schemes. Pressure is displayed by color, density by 29 contours (0.52 to 1.92 step 0.05$)$ and velocity by arrows. For all cases the computations were done and are presented on the square $(x, y) \in(0,1) \times(0,1)$. 
Case 6: The contact resolving ability of WAFT and PPM show up very well here.

All the schemes have grid aligned artefacts in the high pressure areas around boundaries which appear also in density and only for CFLFh also shows up on lower right in the chosen density contour levels. Some of these relicts are standing around original jump segments while the others result from waves emanated from the initial jumps which are faster than the main contact waves.

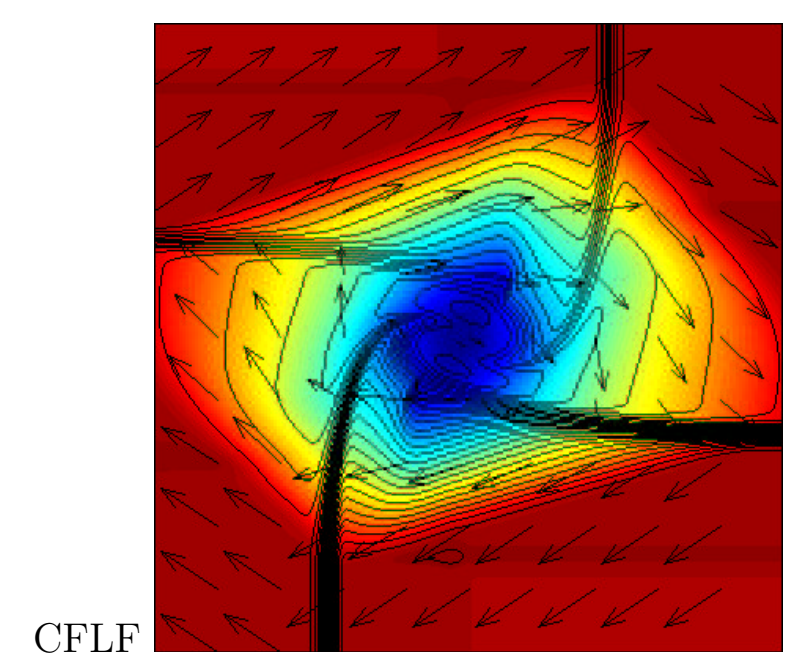

WAFT

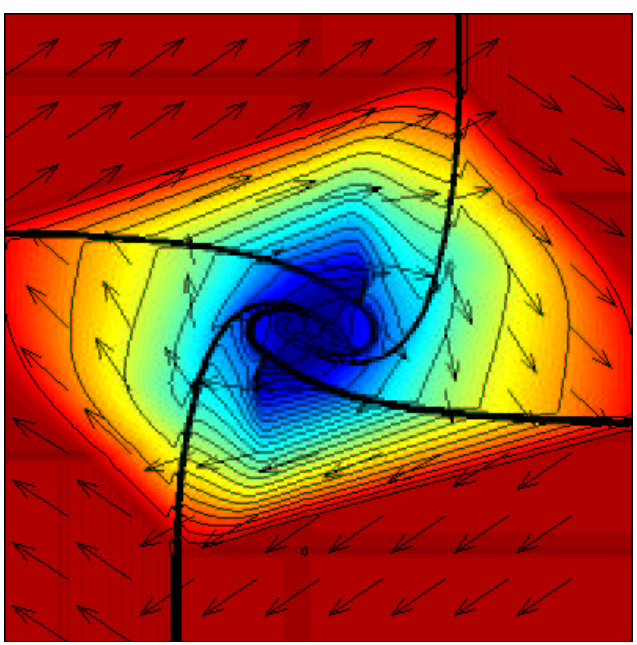

CFLFh

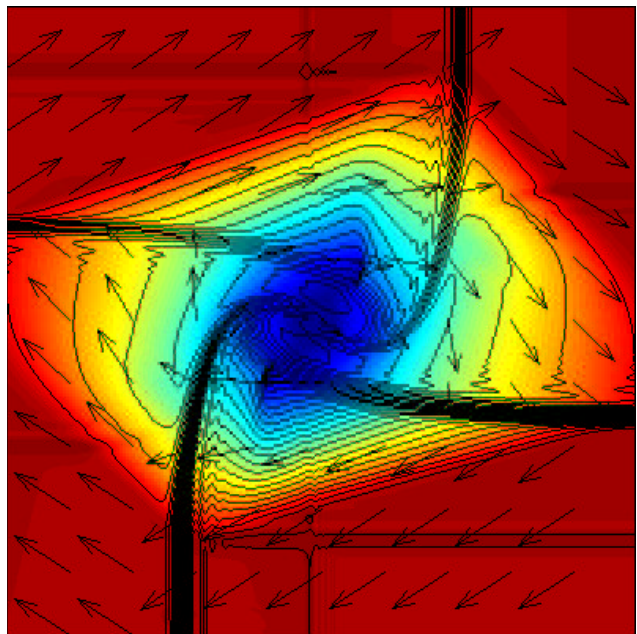

WAFC

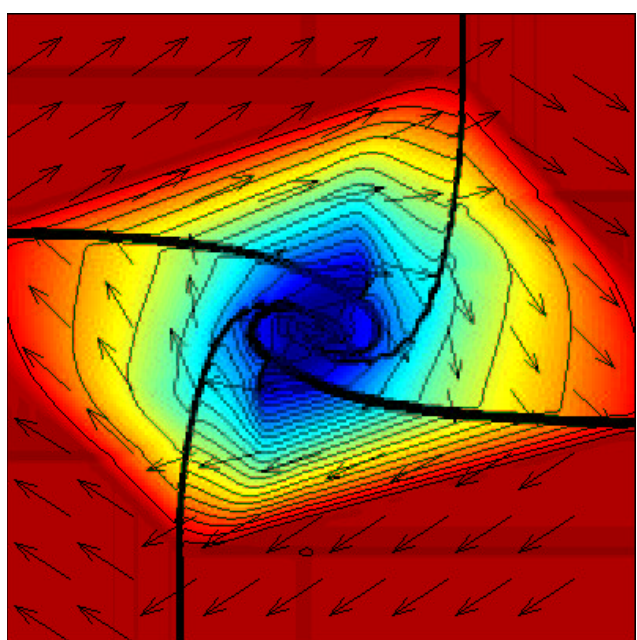

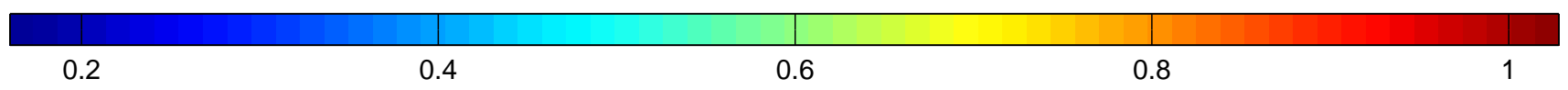

Figure 17: Results for the 2D Riemann problem case 6 by four schemes with pressure colorbar. Pressure is displayed by color, density by 29 contours ( 0.25 to 3.05 step 0.1$)$ and velocity by arrows. For all cases the computations were done and are presented on the square $(x, y) \in(0,1) \times(0,1)$. 


\section{Case 6}
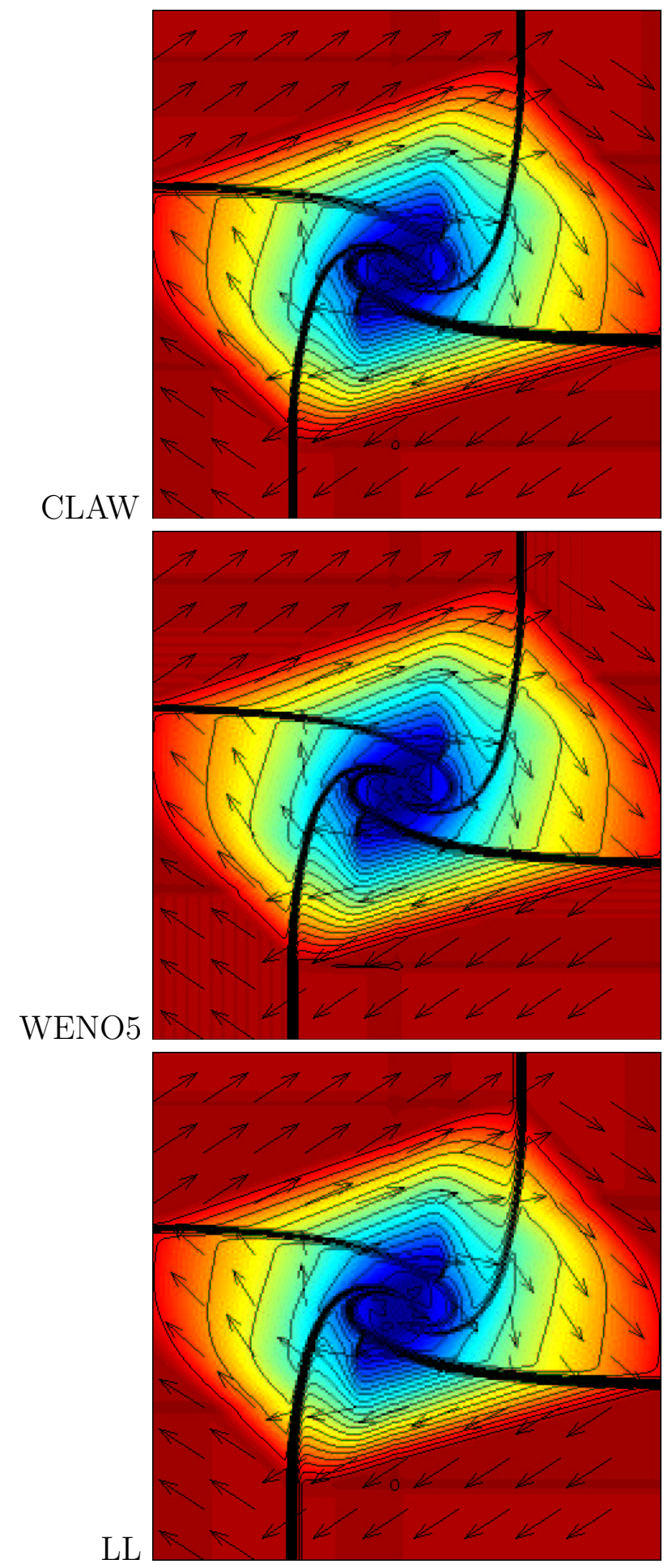

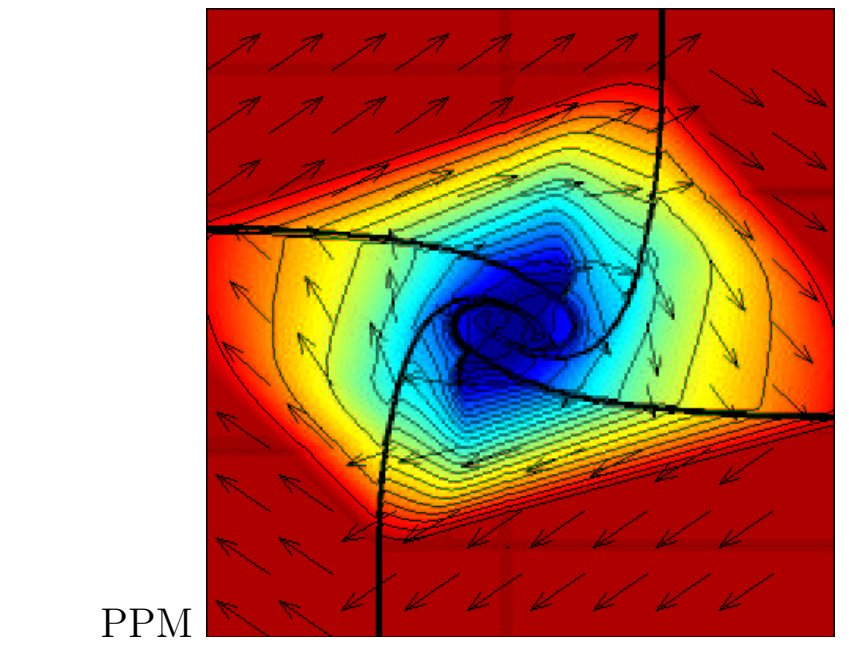

CWENO3
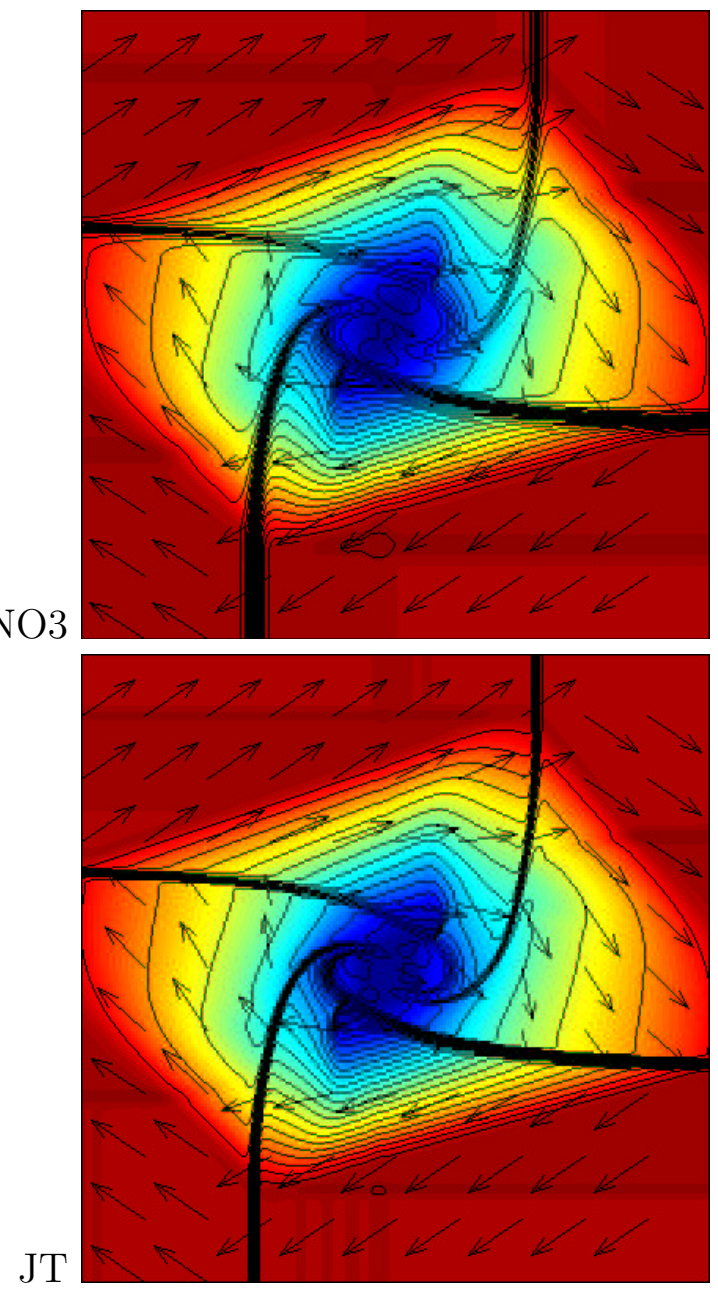

Figure 18: Results for the 2D Riemann problem case 6 by remaining six schemes. Pressure is displayed by color, density by 29 contours ( 0.25 to 3.05 step 0.1$)$ and velocity by arrows. For all cases the computations were done and are presented on the square $(x, y) \in(0,1) \times(0,1)$. 
Case 12: The key issue here is the resolution of the stationary contacts bordering the lower left quadrant. When we compare with the $1 \mathrm{D}$ test 5 we see that all schemes exactly resolving stationary contact there (WAFT, WAFC, CLAW, PPM, WENO5) also resolve exactly the stationary contacts here.

The data for this problem is symmetric about the $(0,0)(1,1)$ diagonal, and the non-symmetric dimensionally split schemes WAFC and PPM preserve the symmetry quite well as all as do the other non-split methods.
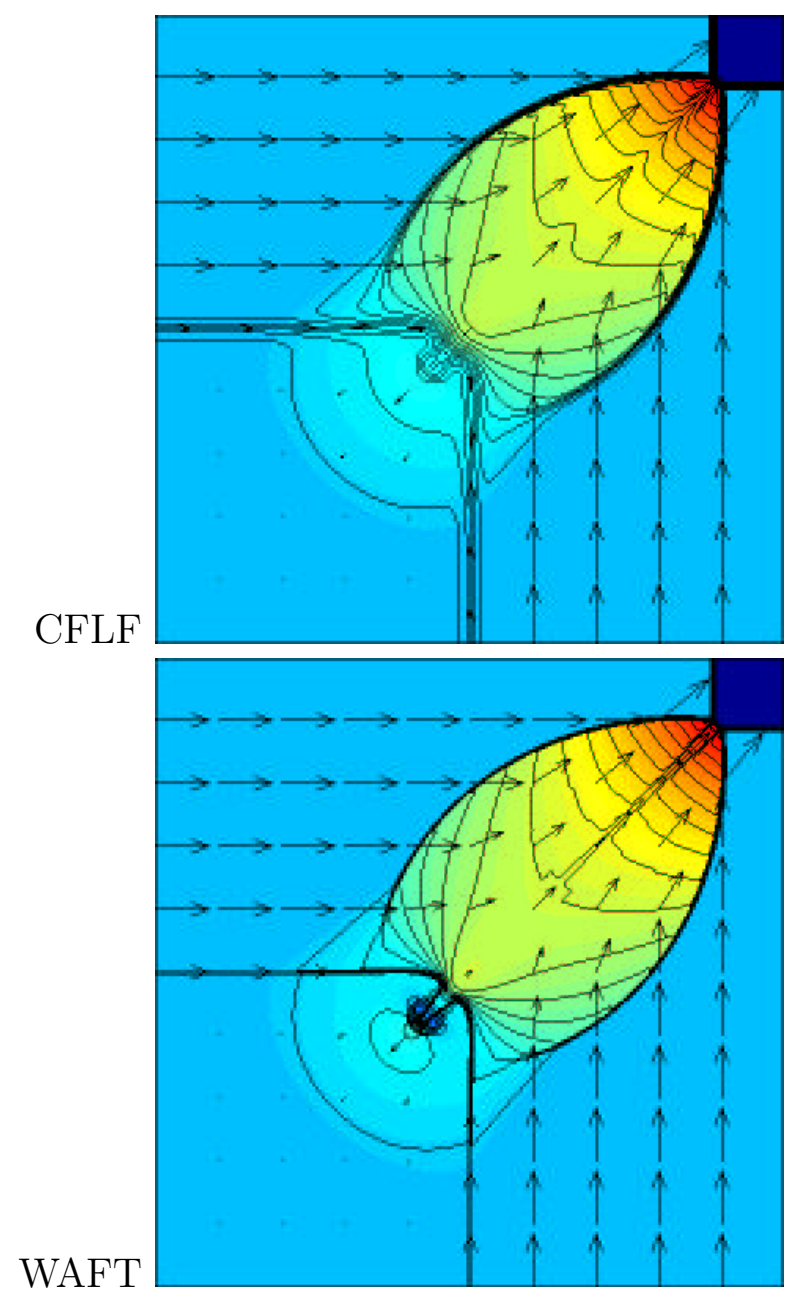

CFLFh

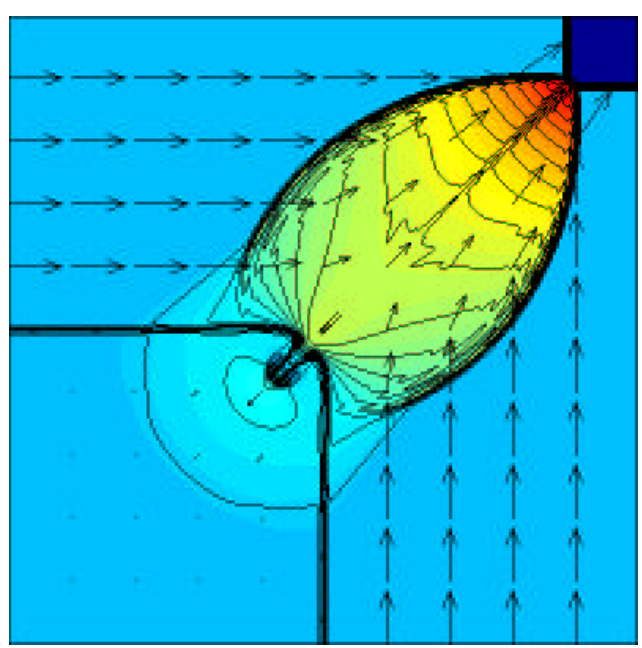

WAFC
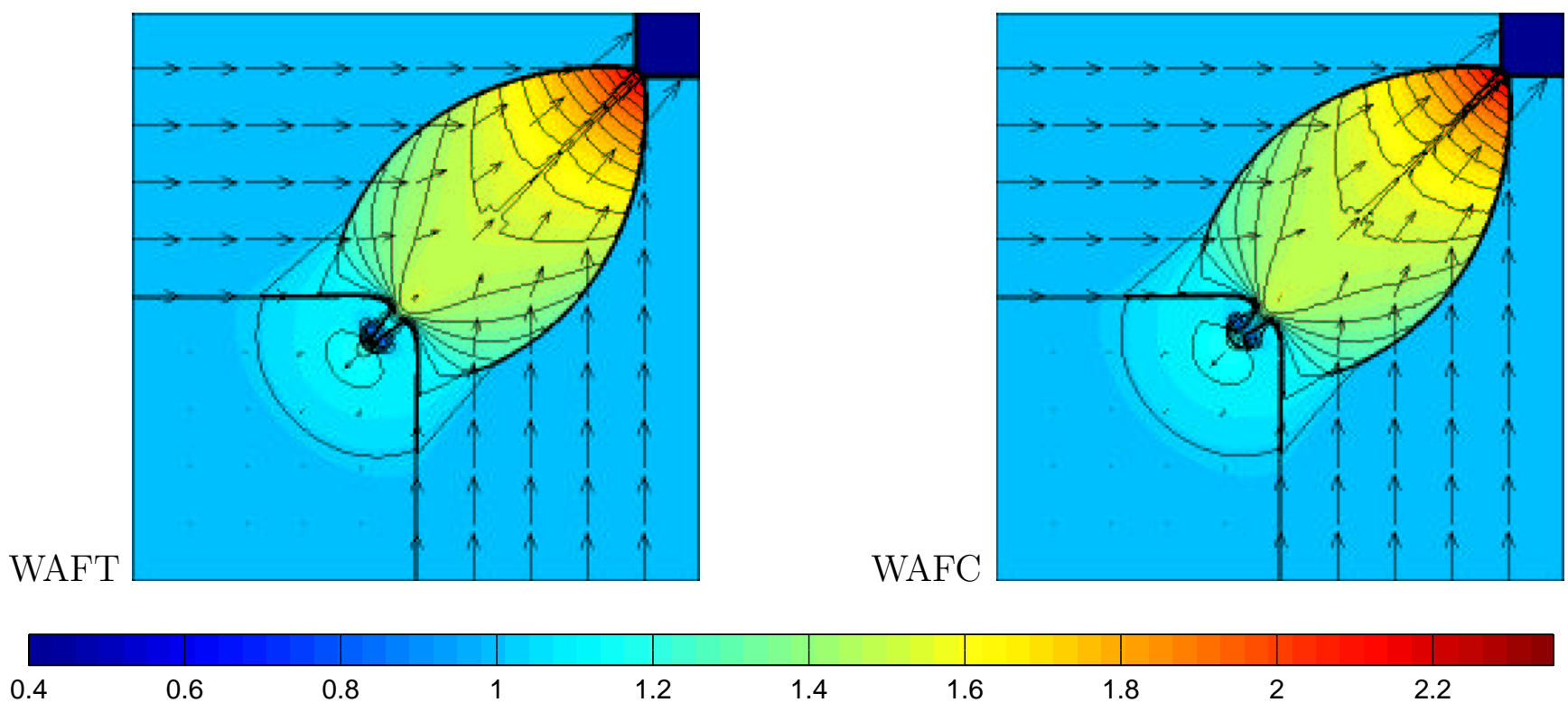

Figure 19: Results for the 2D Riemann problem case 12 by four schemes with pressure colorbar. Pressure is displayed by color, density by 30 contours ( 0.54 to 1.7 step 0.04 ) and velocity by arrows. For all cases the computations were done and are presented on the square $(x, y) \in(0,1) \times(0,1)$. 


\section{Case 12}
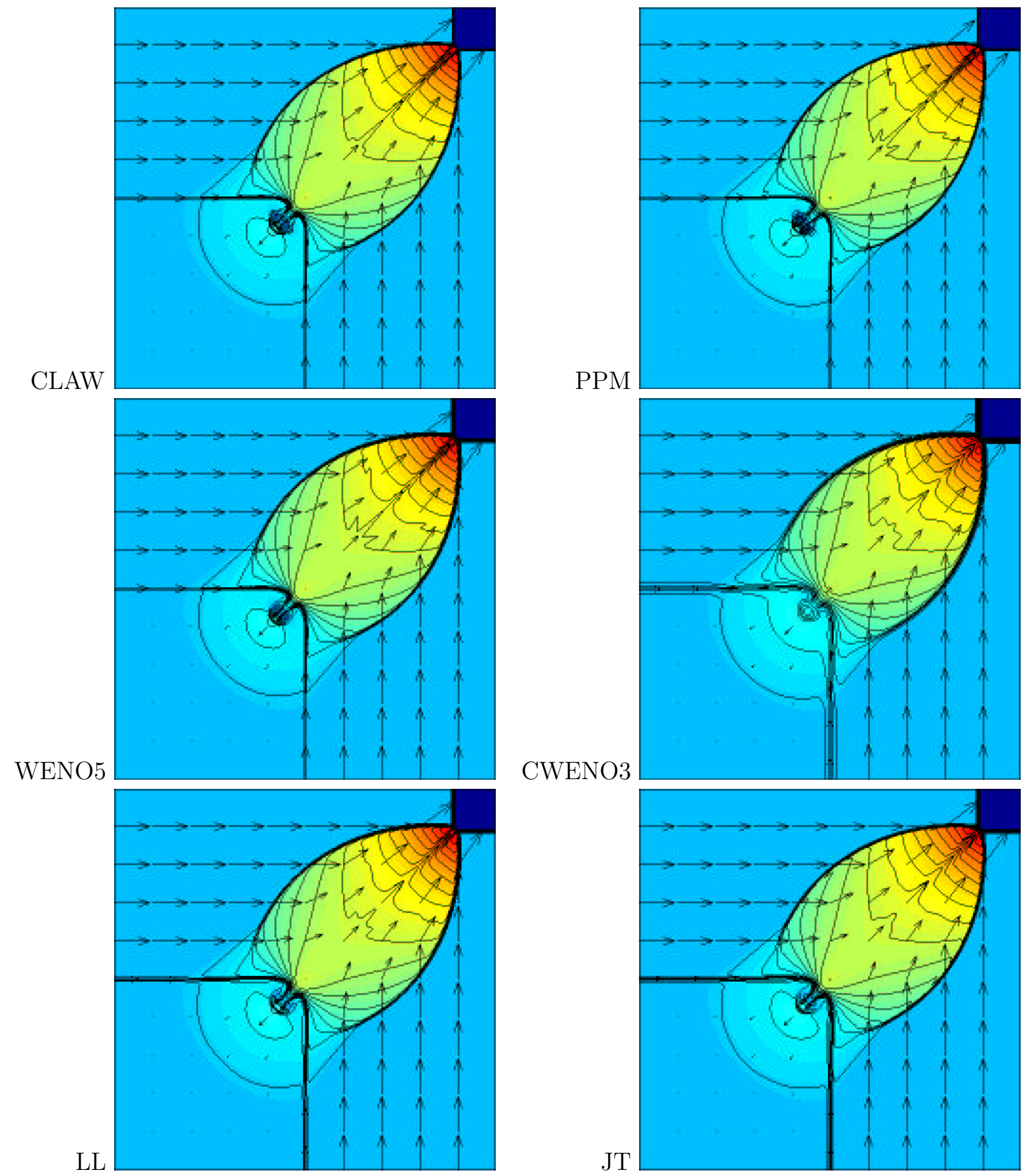

Figure 20: Results for the 2D Riemann problem case 12 by remaining six schemes. Pressure is displayed by color, density by 30 contours (0.54 to 1.7 step 0.04 ) and velocity by arrows. For all cases the computations were done and are presented on the square $(x, y) \in(0,1) \times(0,1)$. 
Case 15: Resolution of slowly moving contacts bordering the lower left constant state is importat here (note the short curved contact in the middle) and one might compare with 1D test 6.

As in case 6 a detailed inspection reveals errors aligned with the slip and shock lines that show up in the pressure color map. As in case 6 these artefacts appear also in density, but they are not visible with the chosen density contour levels, and they have the same origin as in case 6 .

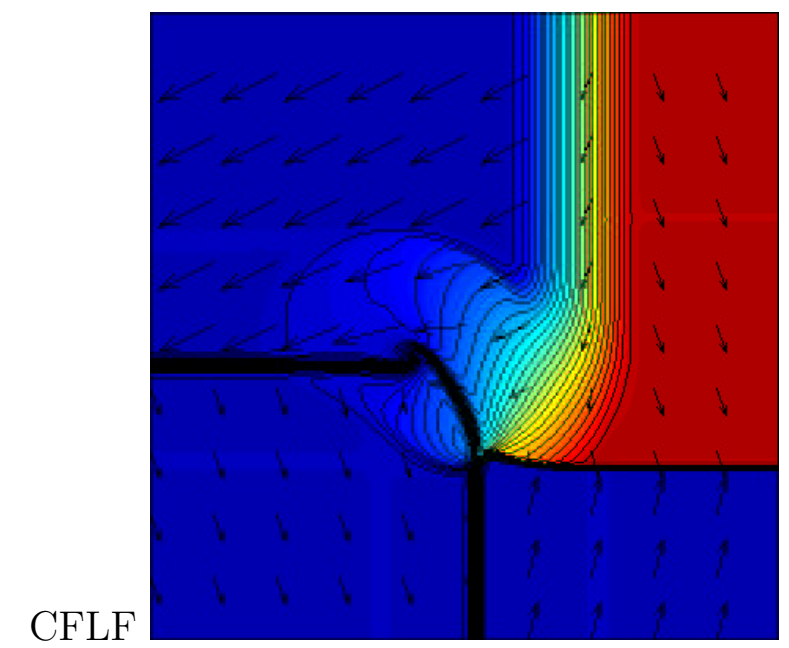

WAFT

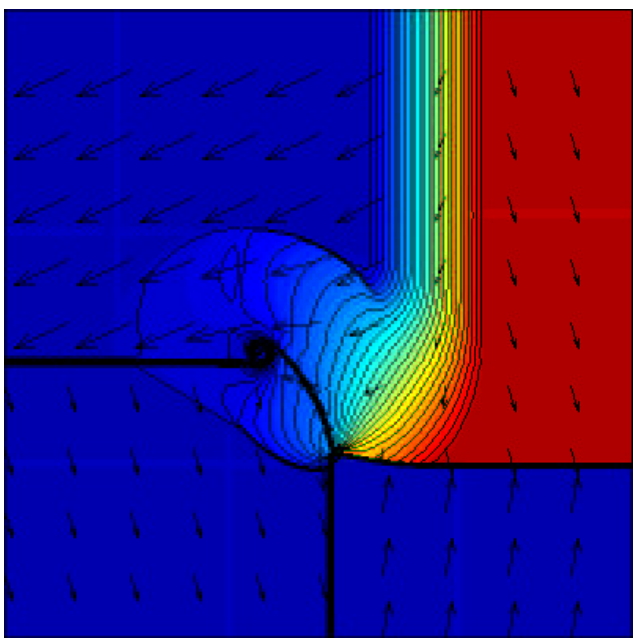

\section{CFLFh}

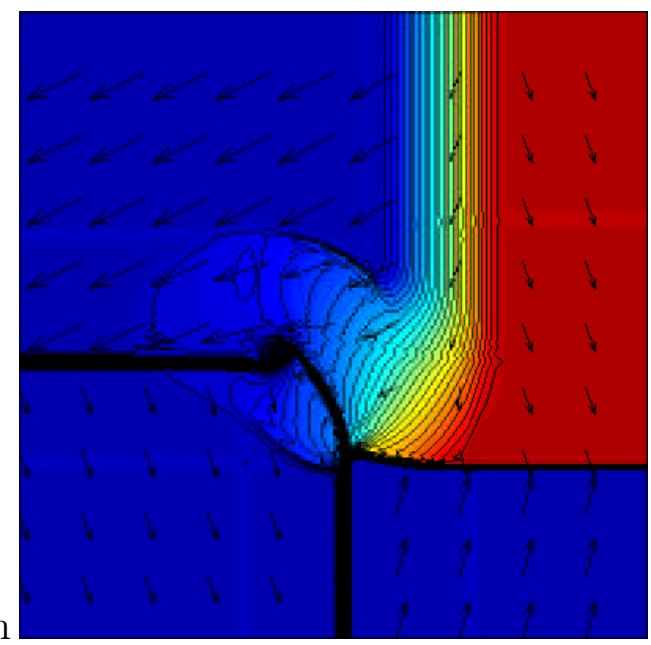

WAFC

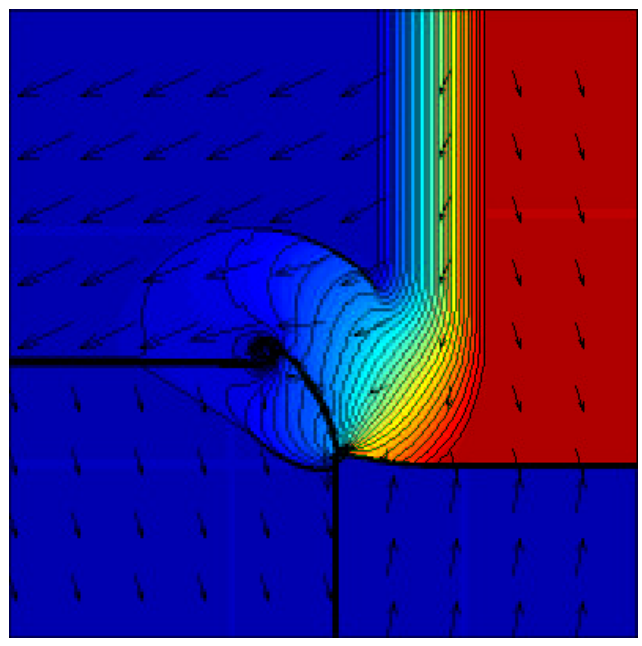

0.4

0.5

0.6

0.7

0.8

0.9

Figure 21: Results for the 2D Riemann problem case 15 by four schemes with pressure colorbar. Pressure is displayed by color, density by 29 contours (0.43 to 0.99 step 0.02 ) and velocity by arrows. For all cases the computations were done and are presented on the square $(x, y) \in$ $(0,1) \times(0,1)$. 


\section{Case 15}
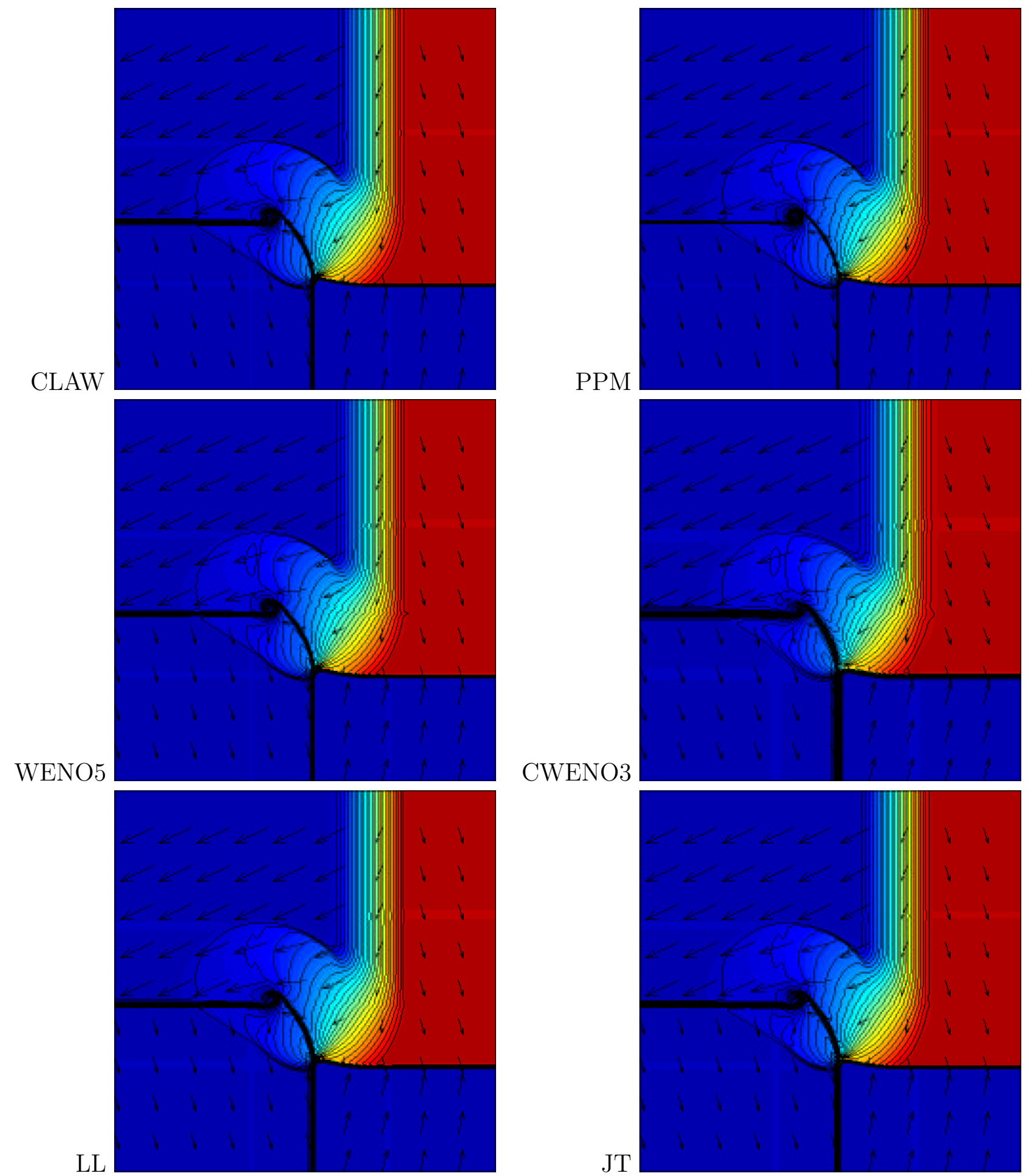

Figure 22: Results for the 2D Riemann problem case 15 by remaining six schemes. Pressure is displayed by color, density by 29 contours (0.43 to 0.99 step 0.02$)$ and velocity by arrows. For all cases the computations were done and are presented on the square $(x, y) \in(0,1) \times(0,1)$. 
Case 17: Here we have an interesting disagreement. LL shows a possibile instability on the lower slip line not seen by the others.

As in case 12 important is the resolution of two standing contacts on the line $x=1 / 2$ and we can again compare with $1 \mathrm{D}$ test 5 .

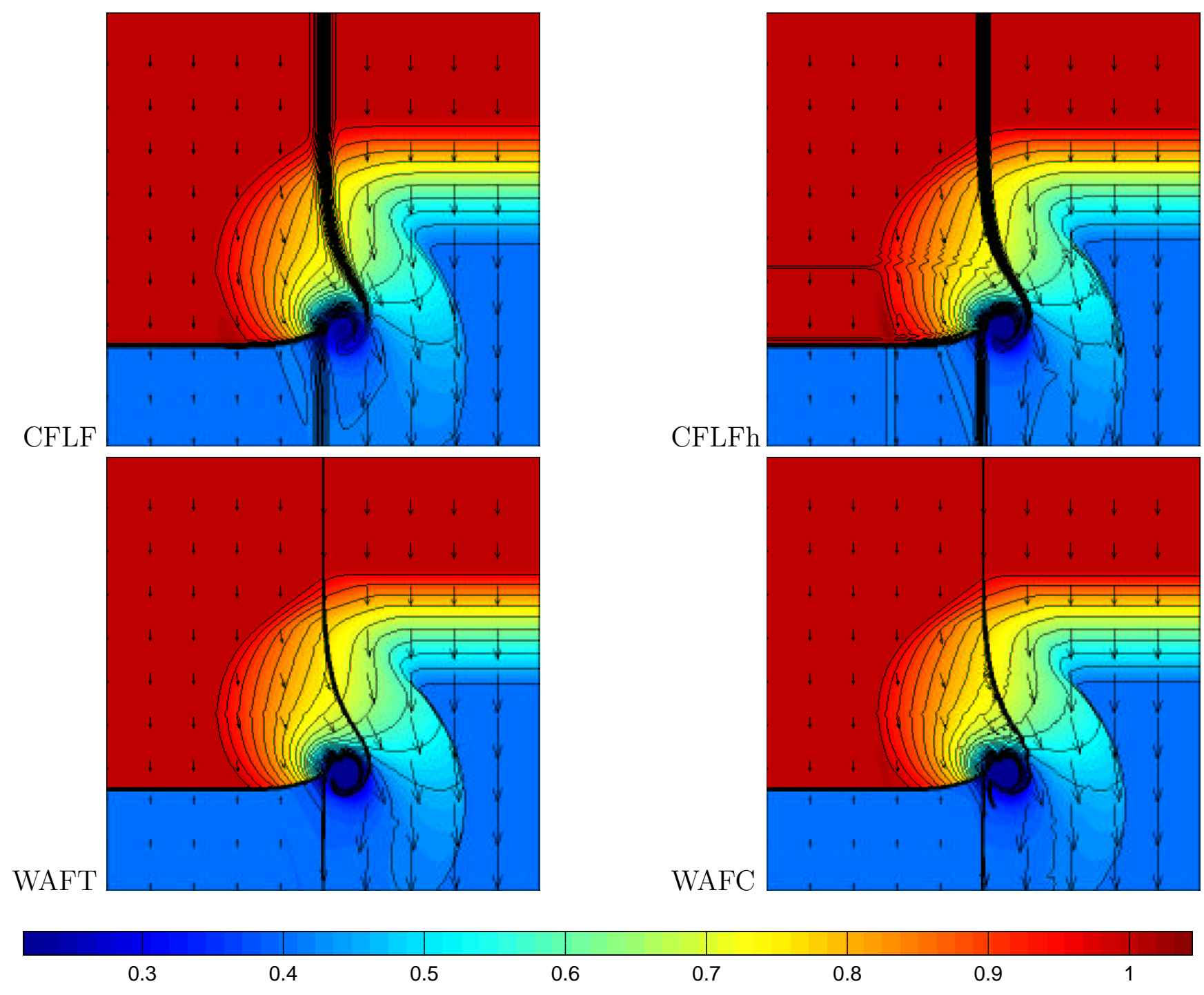

Figure 23: Results for the 2D Riemann problem case 17 by four schemes with pressure colorbar. Pressure is displayed by color, density by 30 contours ( 0.53 to 1.98 step 0.05 ) and velocity by arrows. For all cases the computations were done and are presented on the square $(x, y) \in$ $(0,1) \times(0,1)$. 


\section{Case 17}
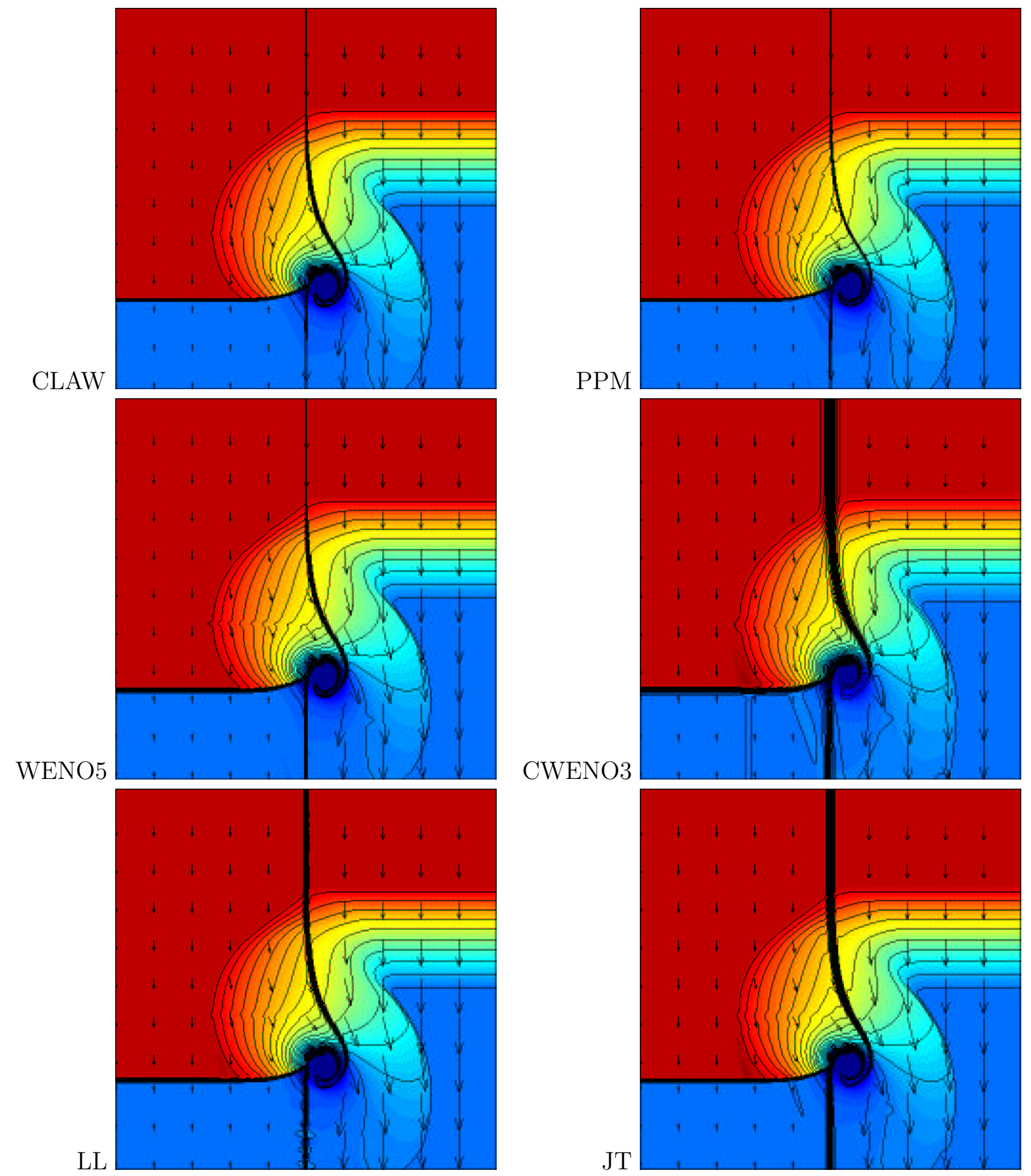

CWENO3

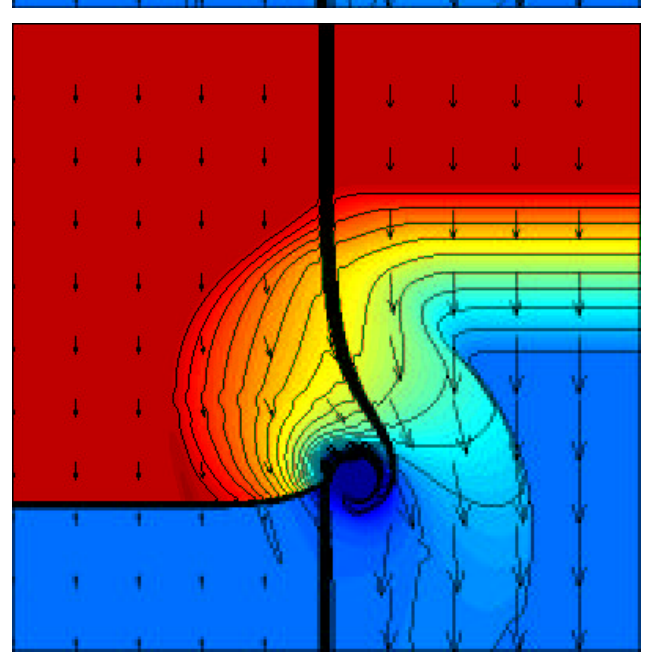

Figure 24: Results for the 2D Riemann problem case 17 by remaining six schemes. Pressure is displayed by color, density by 30 contours (0.53 to 1.98 step 0.05 ) and velocity by arrows. For all cases the computations were done and are presented on the square $(x, y) \in(0,1) \times(0,1)$. 


\subsection{Noh problem}

This is a classic test of W. Noh [38] for an ideal gas with $\gamma=5 / 3$ for which there is an exact solution. The initial density is 1 , the initial pressure is 0 (we set initial pressure to $10^{-6}$ in the numerics as many schemes cannot deal with zero pressure), and the initial velocities are directed toward the origin in the plane with magnitude 1 . The solution is an infinite strength circularly symmetric shock reflecting from the origin. Behind the shock (i.e. inside the circle) the density is 16 , the velocity is 0 and the pressure is $16 / 3$. The shock speed is $1 / 3$ and ahead of the shock, that is for $\sqrt{x^{2}+y^{2}}>t / 3$, the density is $\left(1+t / \sqrt{x^{2}+y^{2}}\right)$ while velocity and pressure remain same as initially. The computational domain is $0 \leq x \leq 1,0 \leq y \leq 1$. At the boundaries $x=1$ and $y=1$ we used the exact density as a function of time and radius together with the initial pressure and velocity. At the other two boundaries $x=0$ and $y=0$ we used the symmetric (reflecting) BCs. This is a difficult problem. The Lagrangian codes dealing with this problem suffer from a very large error in the density at the center. For an analysis of this problem see [45].

Here 1 you can see the animation of $400 \times 400$ cells computation by the CFLF4 scheme to time $T=2$ with frame interval $\Delta T=0.1$.

Those schemes (WAFT, CLAW, WENO5, JT) for which no result is shown failed to run. This seems to be the one case in which CFLF was best among the ten. LL was completely wrong - we also did run it on the whole plane in order to be certain that this was not the result of some error on the symmetry boundary. JT ran on a $100 \times 100$ grid but not on the $400 \times 400$ grid. PPM required a CFL limit of 0.2 for the $400 \times 400$ grid, but ran with $\mathrm{CFL}=0.8$ for a $100 \times 100$ grid.

\footnotetext{
${ }^{1}$ http://www-troja.fjfi.cvut.cz/ liska/CompareEuler/animations/Noh/
} 
Noh
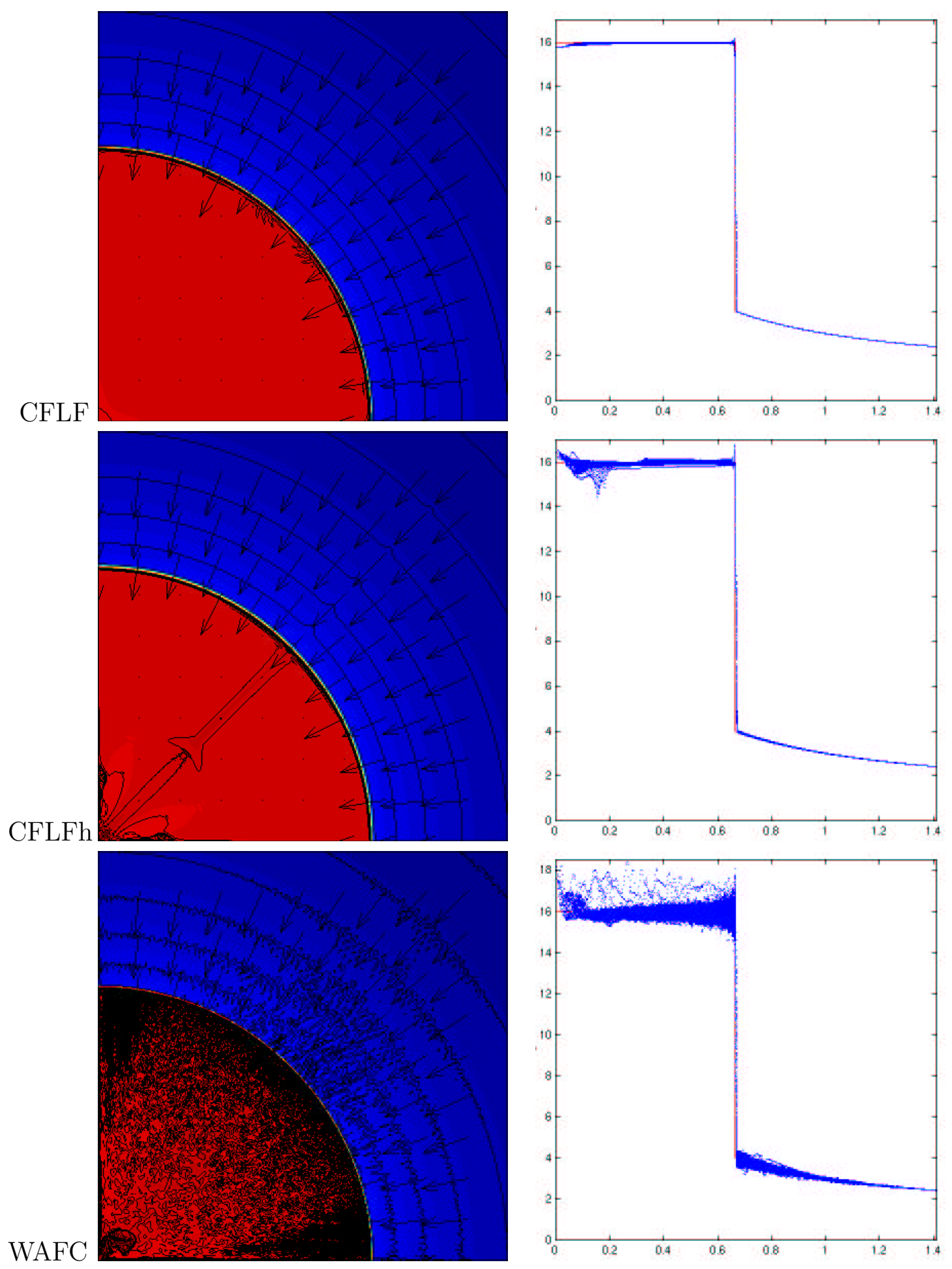

Figure 25: Results for the Noh problem by the CFLF, CFLFh and WAFC schemes. Density color map is overlayed by 23 density contours (2.5 to 4 step 0.25 and 14 to 17 step 0.2 ) and velocity arrows on the left. Scatter plot of density versus radius together with the exact solution is on the right. The computations were done on the square $(x, y) \in(0,1) \times(0,1)$ with $400 \times 400$ grid to time $T=2$. 


\section{Noh}
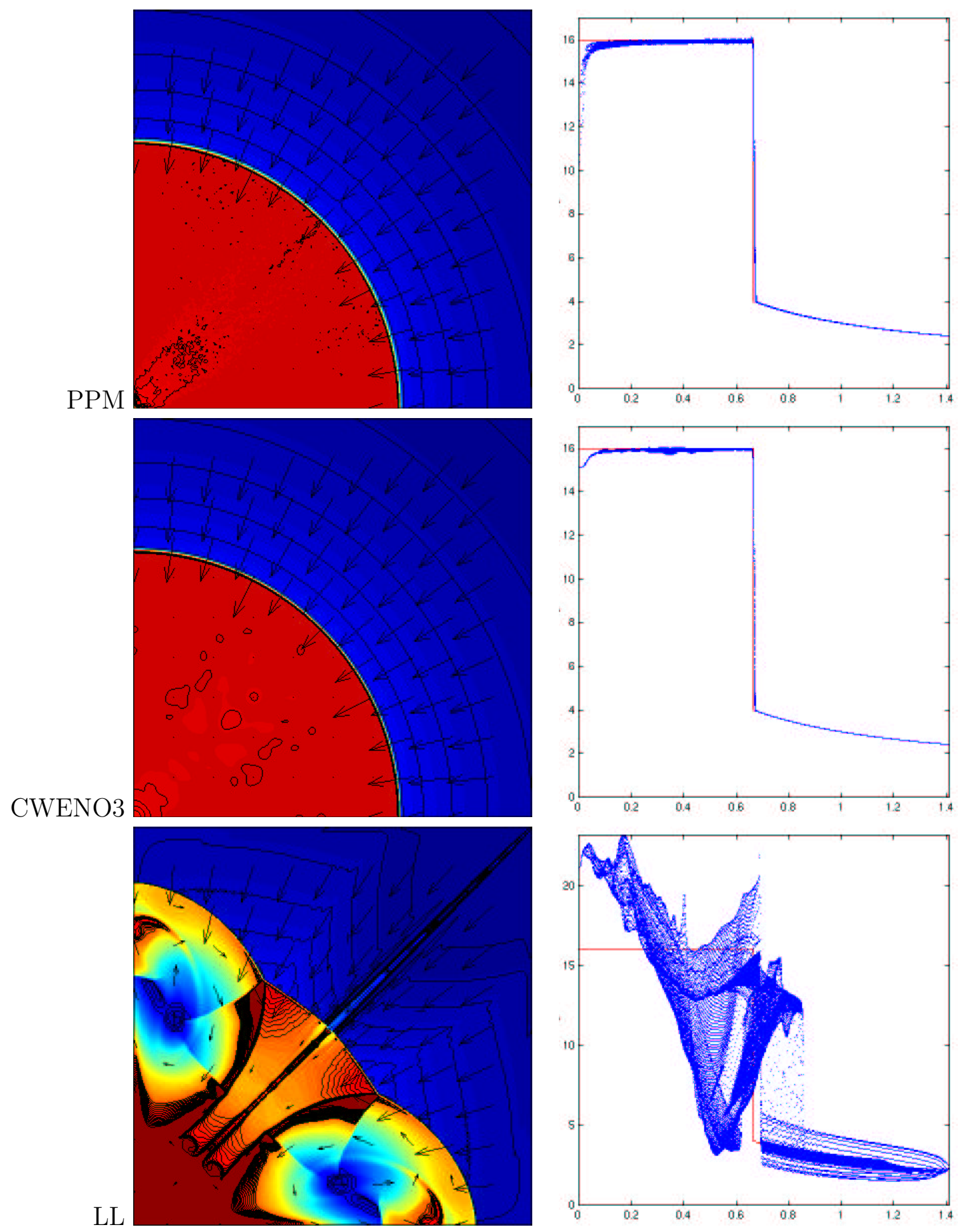

Figure 26: Results for the Noh problem by the PPM, CWENO3 and LL schemes. WAFT, CLAW, WENO5 and JT have failed for this problem. Density color map is overlayed by 23 density contours ( 2.5 to 4 step 0.25 and 14 to 17 step 0.2 ) and velocity arrows on the left. Scatter plot of density versus radius is on the right. The computations were done on the square $(x, y) \in(0,1) \times(0,1)$ with $400 \times 400$ grid to time $T=2$. 


\subsection{Rayleigh-Taylor instability}

Rayleigh-Taylor instability is a physical phenomenon appearing when a layer of heavier fluid is placed on top of a layer of lighter fluid. For this problem we include a gravitational source term in the momentum equation. We treat this problem in the region $(x, y) \in(0,1 / 6) \times(0,1)$ with the gravitational acceleration $g=0.1$ in $-y$ direction. The upper fluid has density 2 and the lower fluid 1. The interface of the fluids is at $y=1 / 2+0.01 \cos (6 \pi x)$, i.e. a slightly perturbed line $y=1 / 2$. The initial pressure is hydrostatic and the fluids are initially at rest. Around the interface the initial conditions are smoothed out. Boundary conditions on all four border lines are reflecting.

Here 2 you can see the animation of the $100 \times 400$ cells computation by the CFLFh hybrid scheme to time $T=8.5$ with frame interval $\Delta T=0.5$.

These runs are for a grid of $100 \times 400$ cells on a half of the mushroom which are then mirrored in the figures. Density color map and density contours are shown separately. The solid line in the contour plot, was provided by J. Grove and V. Mousseau of Los Alamos [46] and is the result of a front tracking code, while the dashed line is the density $\rho=1.5$ contour. The interface between the light and heavy fluid is unstable. As might be expected, the less dissipative schemes such as CLAW and WENO5 show this interface breaking up, while the more dissipative schemes like CFLF suppress the instability. Tariq Aslam [47] has reported to us that the interface breaks up very early with a very high resolution fine grid WENO scheme. The front tracking contour also seems to suppress the instability.

\footnotetext{
${ }^{2}$ http://www-troja.fjfi.cvut.cz/ Iiska/CompareEuler/animations/Rayleigh-Taylor/
} 


\section{Rayleigh-Taylor}

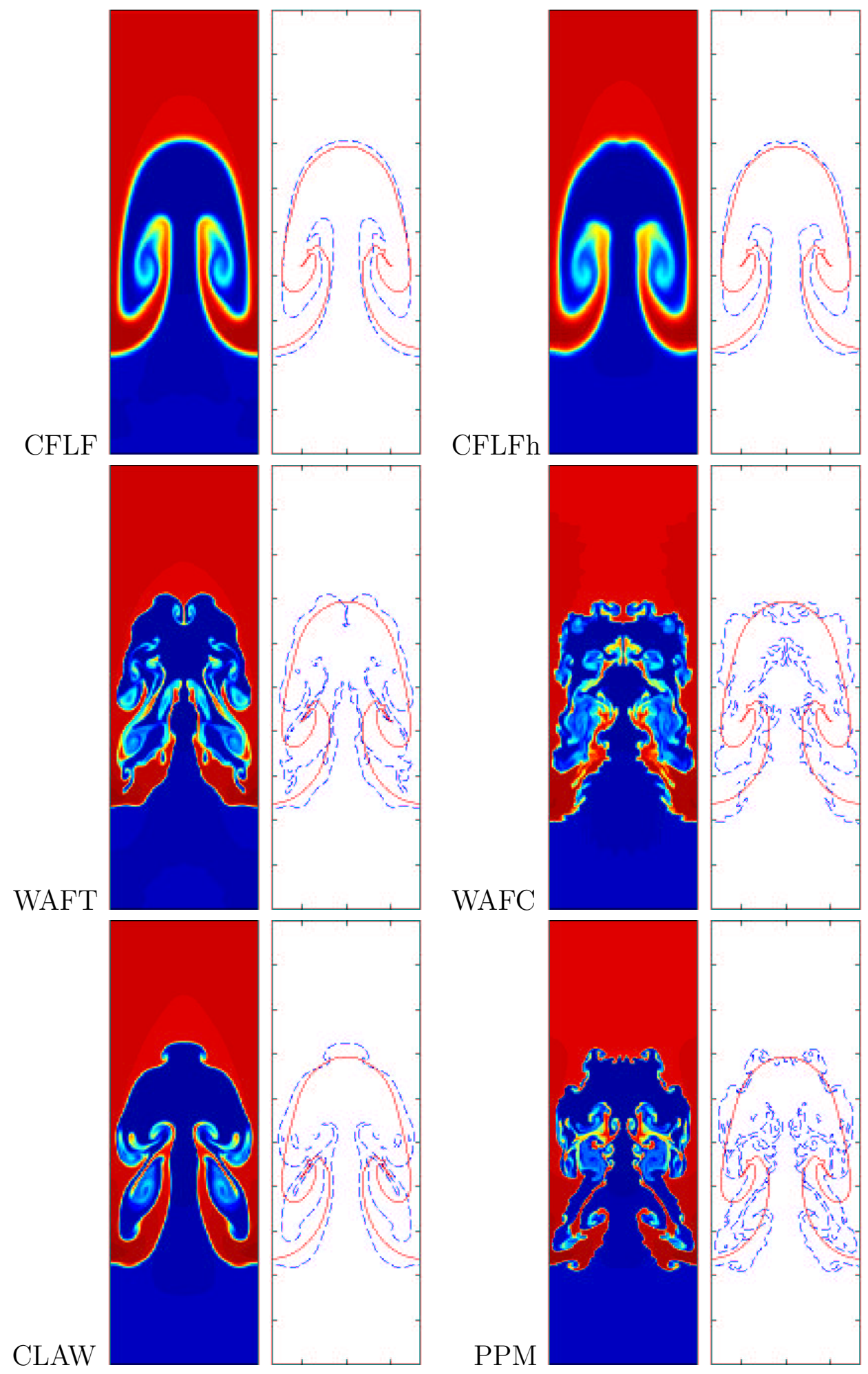

Figure 27: Results for the Rayleigh-Taylor problem by six schemes. Density color map and front contour. The solid line in the contour plots is the result of a front tracking code. The computations were done on the rectangle $(x, y) \in(0,1 / 6) \times(0,1)$ with $100 \times 400$ grid to time $T=8.5$. 


\section{Rayleigh-Taylor}
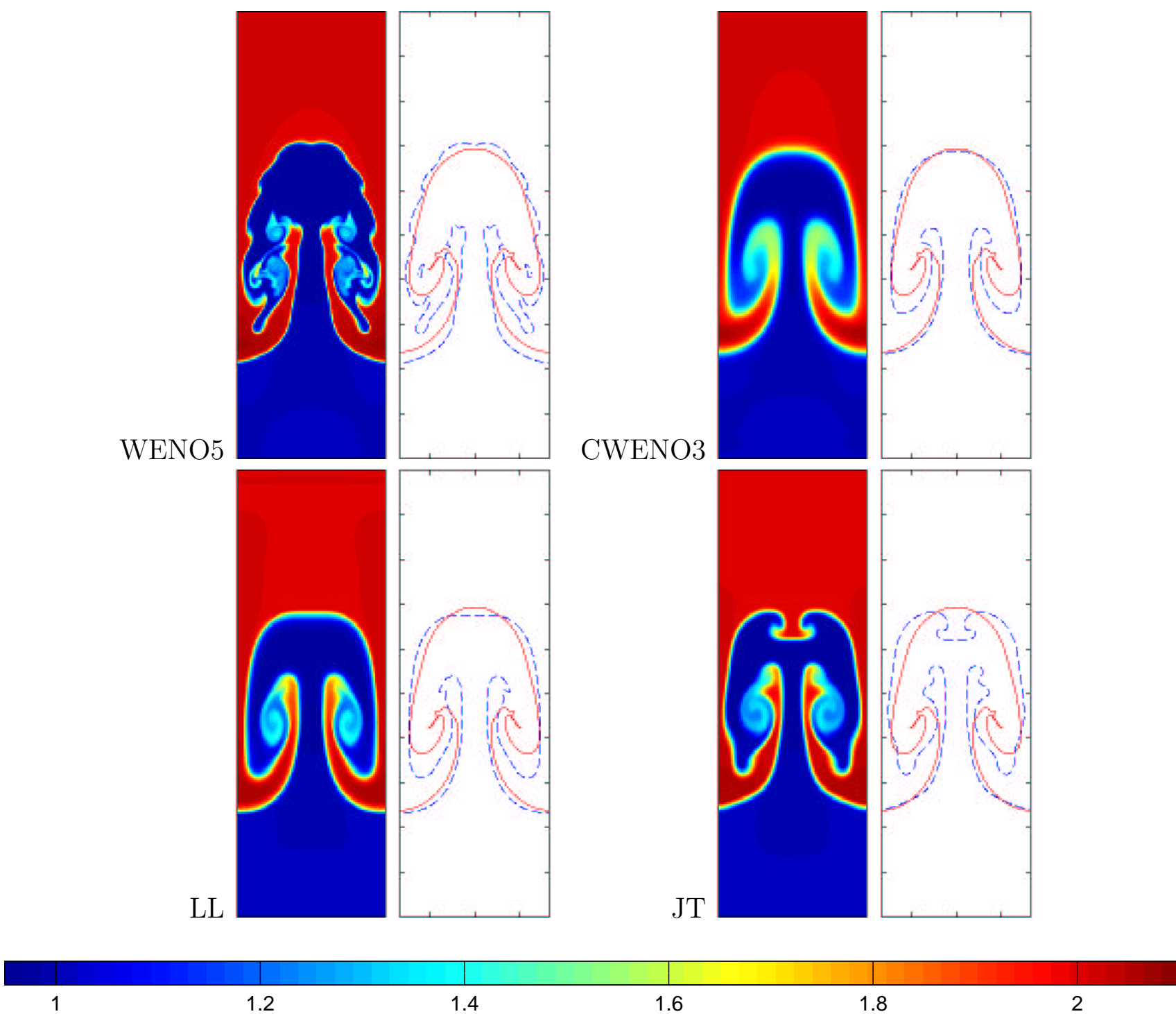

Figure 28: Results for the Rayleigh-Taylor problem by remaining four schemes with density colorbar. Density color map and front contour. The solid line in the contour plots is the result of a front tracking code. The computations were done on the rectangle $(x, y) \in(0,1 / 6) \times(0,1)$ with $100 \times 400$ grid to time $T=8.5$. 


\subsection{Implosion problem}

This converging shock problem has been presented in [48]. In [4.9] several variants of this problem including also other shapes of interior low density and low pressure region have been treated. The gas is placed in a square box. Inside a smaller square centered at the center of the box and rotated by $\pi / 4$ (see Fig. 29) the gas has initialy smaller density and pressure than in the rest of the box. As in [48] we use the box $(x, y) \in(-0.3,0.3) \times(-0.3,0.3)$ and the smaller square with corners at $( \pm 0.15,0),(0, \pm 0.15)$. The computation is done only in the upper right quadrant $(x, y) \in(0,0.3) \times(0,0.3)$ of the box with diamond corners at points $(0.15,0),(0,0.15)$. Initial data inside the diamond are $\rho_{i}=0.125, p_{i}=0.14$ and outside are $\rho_{o}=1, p_{o}=1$. Initial velocities are zero. The gas constant is $\gamma=1.4$. Reflecting boundary conditions are used on all four boundaries.

The initial data are the Sod problem data [9].

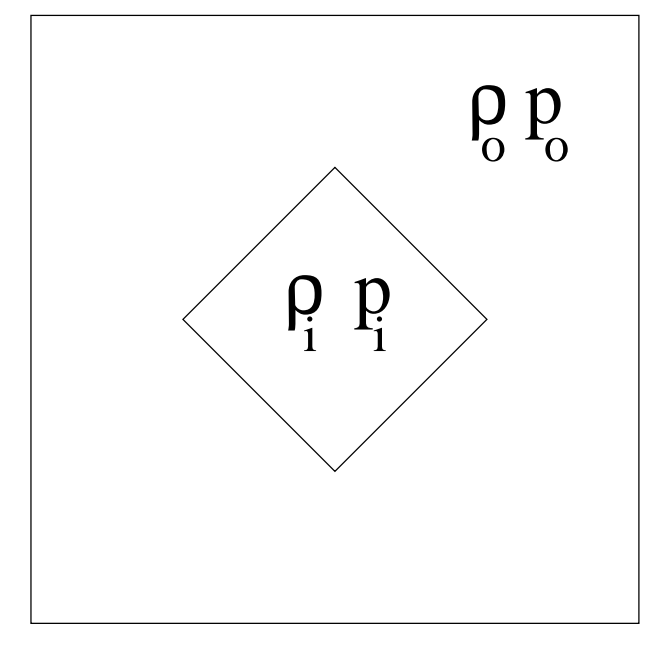

Figure 29: Initial conditions for the diamond problem

Here ${ }^{3}$ you can see the animation of $400 \times 400$ cells computation. by the CLAW scheme to time $T=2.5$ with frame interval $\Delta T=0.05$.

Here H $^{3}$ you can see the early stage animation of $400 \times 400$ cells computation. by the WAFT scheme to time $T=0.1$ with frame interval $\Delta T=0.005$.

There is a clear consensus among the codes for the very early stages of the evolution. The inital interior diamond boundary is a contact discontinuity that is nicely resolved by PPM and CLAW. The color pressure map shows that the pressure is continuous normal to the contact, but also that there is a pressure discontinuity tangential to the contact. The animation is particularly effective in showing the wave structure.

In the later stages there is also a consensus on the gross structure of the waves reflected fromn the boundary. The fate of the initial contact discontinuity is not clear, but there is remarkable

\footnotetext{
${ }^{3}$ http://www-troja.fjfi.cvut.cz/ liska/CompareEuler/animations/implosion/

${ }^{4}$ http://www-troja.fjfi.cvut.cz/ liska/CompareEuler/animations/implosion-short/
} 
agreement between CLAW and WENO5 that a jet has formed.

We can also see here the lack of symmetry in the split dimension PPM and WAFC, which both use alternating Strang splitting, as well as in the 2D code WAFT using superbee limiter (with minmod limiter WAFT is keeping symmetry).

In the following figures we present results for the $400 \times 400$ grid for the implosion problem at early and late stage. The early stage is at time $T=0.045$ while the late one is at $T=2.5$. 


\section{Implosion Early}
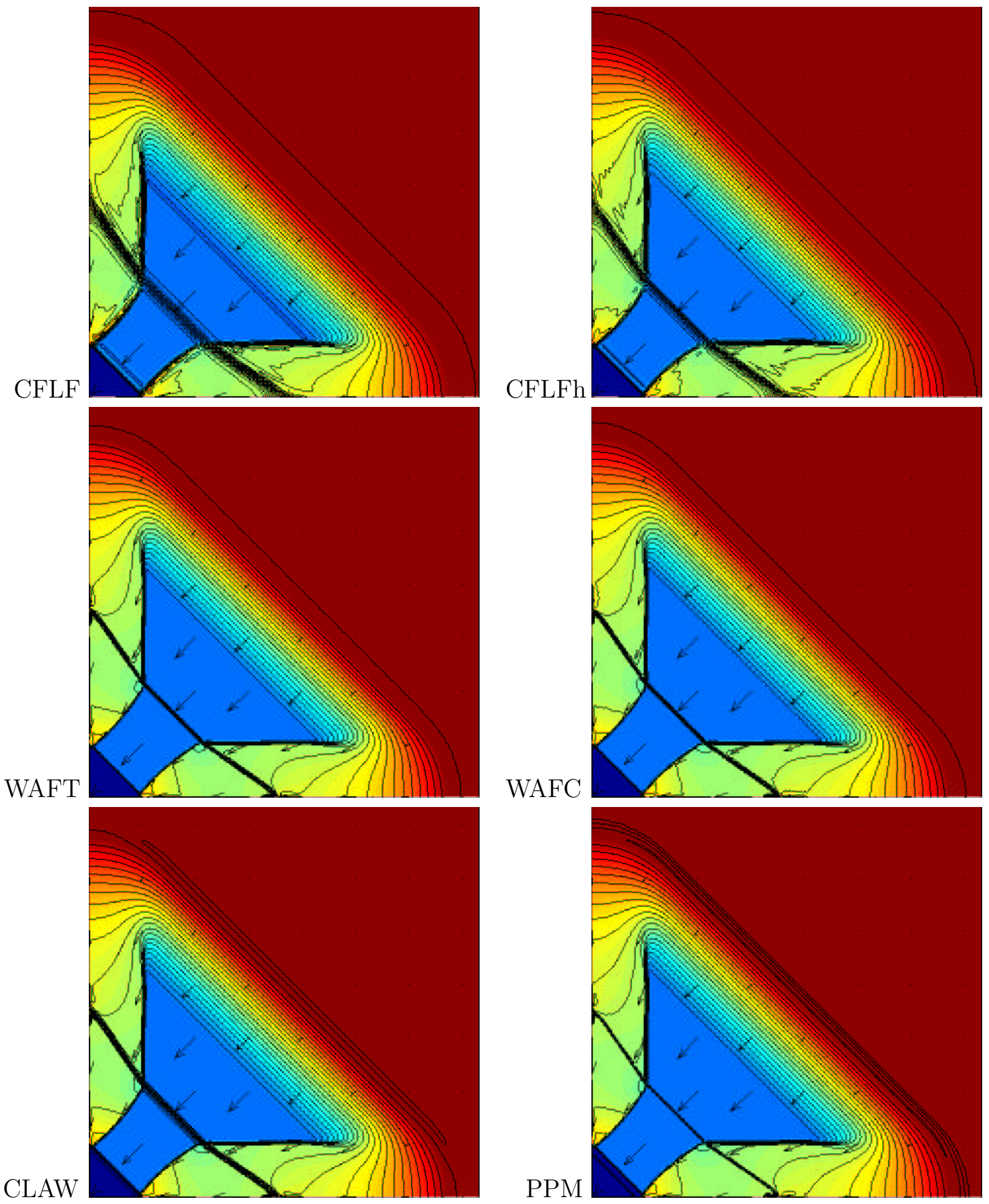

Figure 30: Results for the implosion problem by six schemes. Color pressure map is overlayed by 36 density contours $(0.125$ to 1 step 0.025$)$ and velocity arrows. The computations were done on the square $(x, y) \in(0,0.3) \times(0,0.3)$ with $400 \times 400$ grid to time $T=0.045$ and is presented on the square $(0,0.22) \times(0,0.22)$. 


\section{Implosion Early}

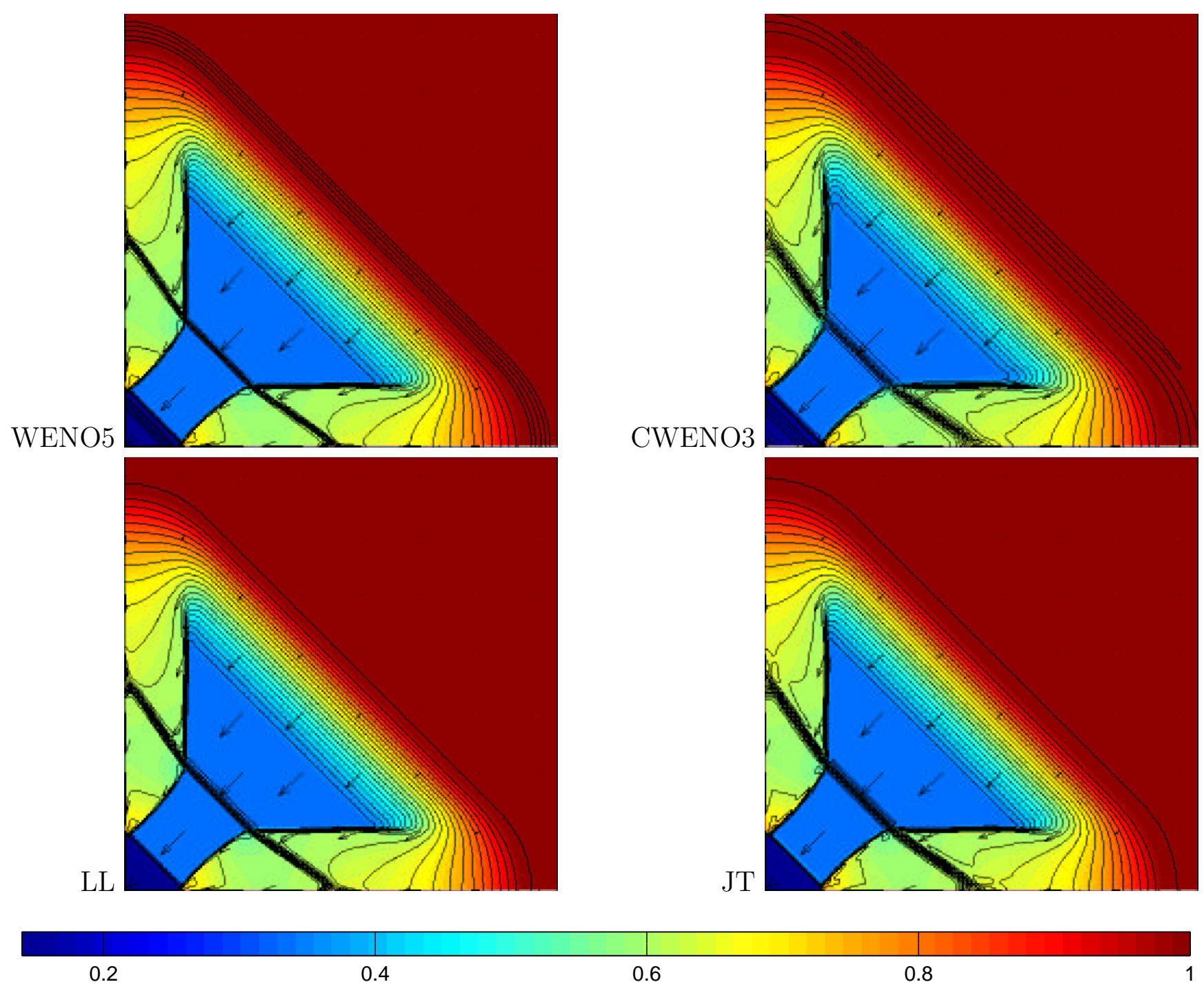

Figure 31: Results for the implosion problem by remaining four schemes with pressure colorbar. Color pressure map is overlayed by 36 density contours (0.125 to 1 step 0.025$)$ and velocity arrows. The computations were done on the square $(x, y) \in(0,0.3) \times(0,0.3)$ with $400 \times 400$ grid to time $T=0.045$ and is presented on the square $(0,0.22) \times(0,0.22)$. 


\section{Implosion Late}
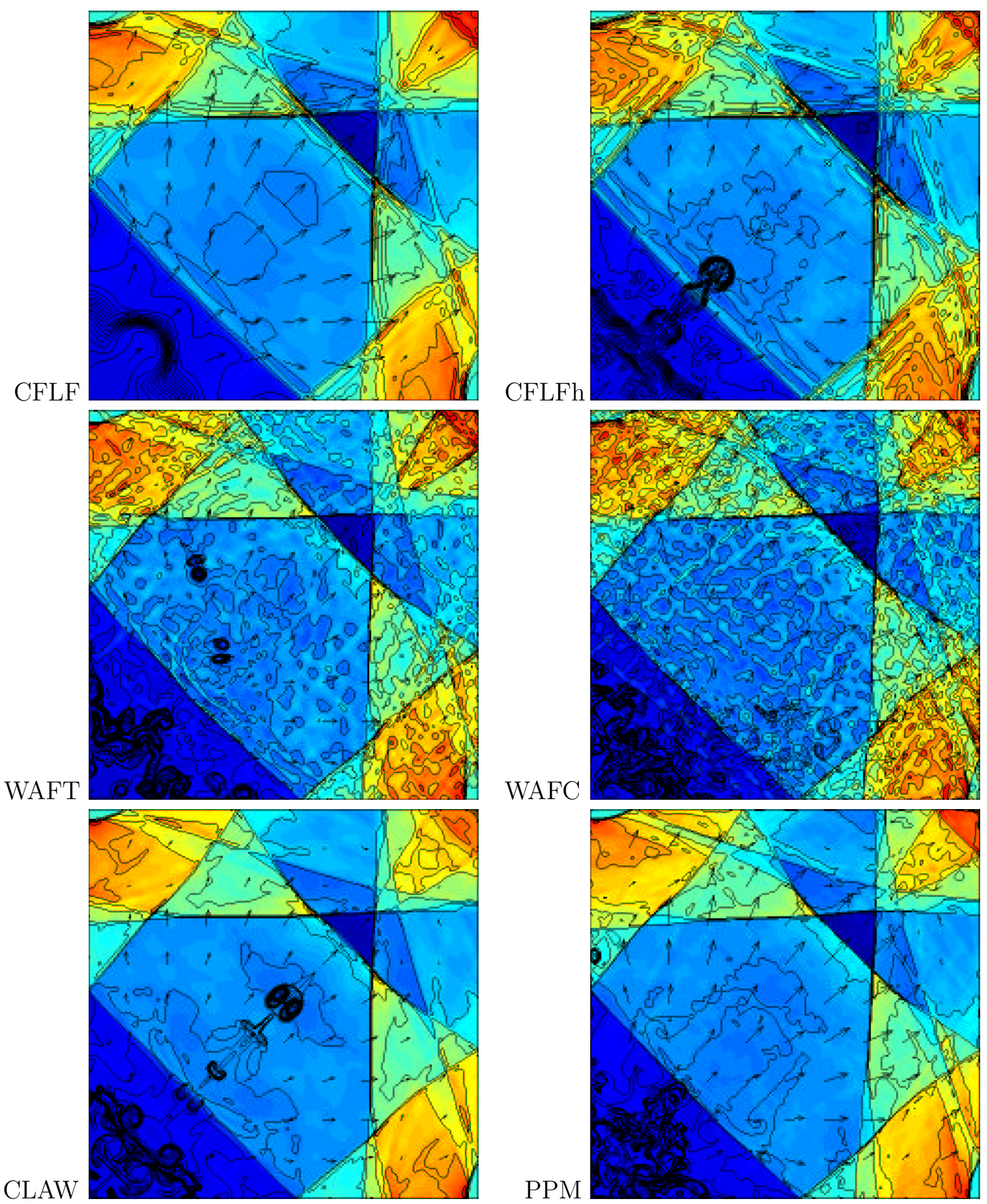

Figure 32: Results for the implosion problem by six schemes. Color pressure map is overlayed by 31 density contours ( 0.35 to 1.1 step 0.025$)$ and velocity arrows. The computations were done on the square $(x, y) \in(0,0.3) \times(0,0.3)$ with $400 \times 400$ grid to time $T=2.5$. 


\section{Implosion Late}

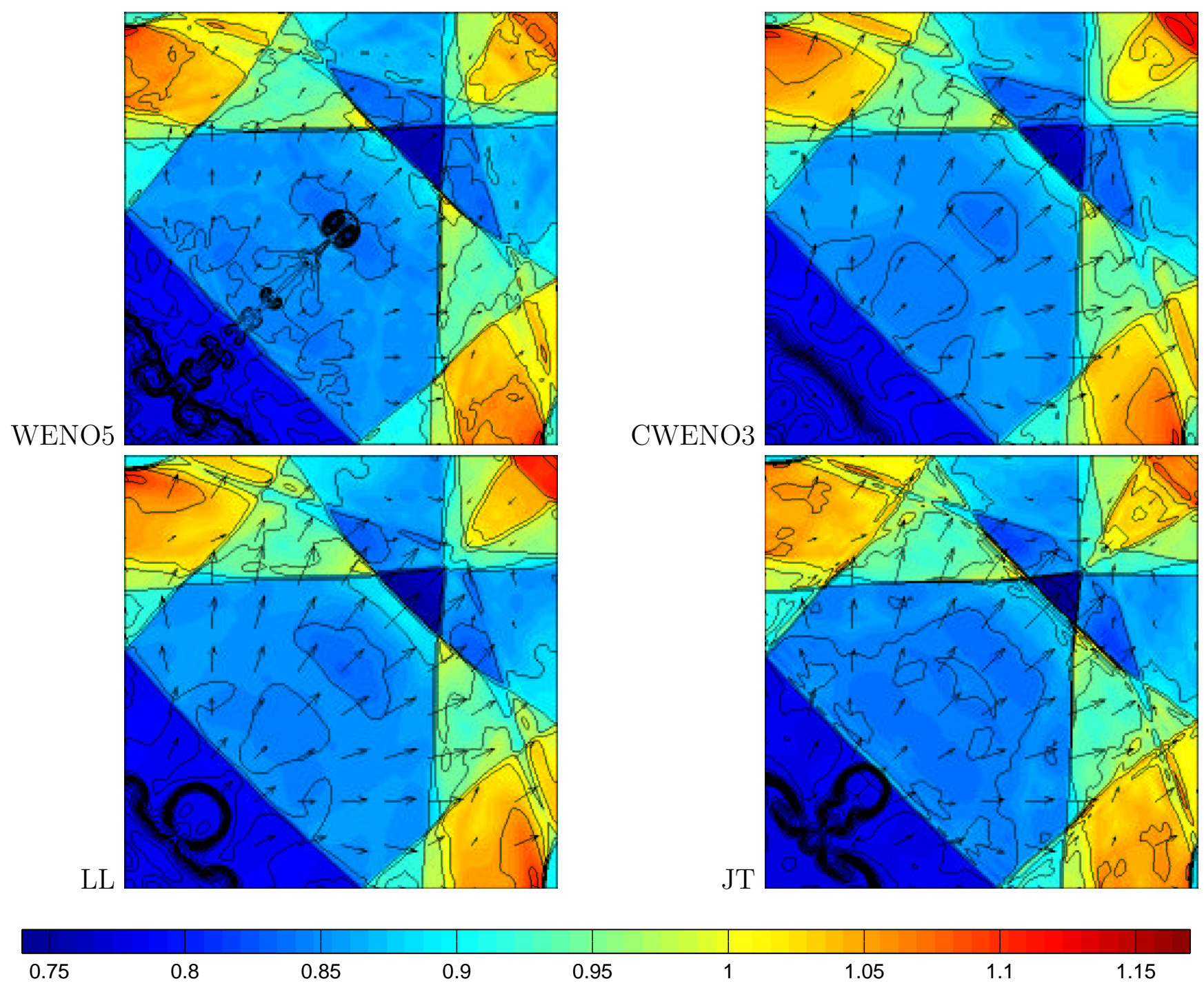

Figure 33: Results for the implosion problem by remaining four schemes with pressure colorbar. Color pressure map is overlayed by 31 density contours ( 0.35 to 1.1 step 0.025$)$ and velocity arrows. The computations were done on the square $(x, y) \in(0,0.3) \times(0,0.3)$ with $400 \times 400$ grid to time $T=2.5$. 


\subsection{Explosion}

The explosion problem proposed in [10] is a circularly symmetric 2D problem with initial circular region of higher density and higher pressure. In particular we set the center of the circle to the origin, its radius to 0.4 and compute on a quadrant $(x, y) \in(0,1.5) \times(0,1.5)$. Density and pressure are $\rho_{i}=1, p_{i}=1$ inside the circle and $\rho_{o}=0.125, p_{o}=0.1$ outside. The gas is initially at rest and its gas constant is $\gamma=1.4$. This problem (evolution of unstable contact at later times) is sensitive to perturbations of the interface and as noted in [10] for the cells which are crossed by the initial interface circle one needs to use area weighted initial density and pressure.

Here 9 you can see an animation of $400 \times 400$ cells computation by the WAFT scheme to time $T=3.5$ with frame interval $\Delta T=0.1$. This animation uses area averaged initial data for those cells crossed by the circle. The frames have pressure colormap with density contours on the left and density colormap with pressure contours on the right to distinguish unstable circular contact. The color scale is different for each frame so that the same colors on different frames do not correspond to the same value.

Here ${ }^{6}$ you can see an animation of $400 \times 400$ cells computation by the WAFT scheme till $T=3.5$ with frame interval $\Delta T=0.1$. This animation does not have the smoothed initial interface. The frames have pressure colormap with density contours on the left and density colormap with pressure contours on the right to distinguish the unstable circular contact. The colormaps use for each frame a different interval of pressure and/or density values, so that the same colors in different frames do not correspond to the same value.

This proceeds as described in [10]. The initial data at the contact have been smoothed by area weighting as suggested in [10], nevertheless it appears that an instability develops. There is a shock reflecting from the center that passes through the contact and seems to have no effect.

There are numerical boundary effects at the upper right corner that we were not able to eliminate. Different schemes resolve density and pressure in different intervals, the most visible difference being for CWENO3.

These runs below are for grids 400 by 400 cells at time $T=3.2$. A color pressure map is overlayed by density contours and velocity arrows.

\footnotetext{
${ }^{5}$ http://www-troja.fjfi.cvut.cz/ liska/CompareEuler/animations/explosion/

${ }^{6}$ http://www-troja.fjfi.cvut.cz/ liska/CompareEuler/animations/explosion-noweight/
} 


\section{Explosion}
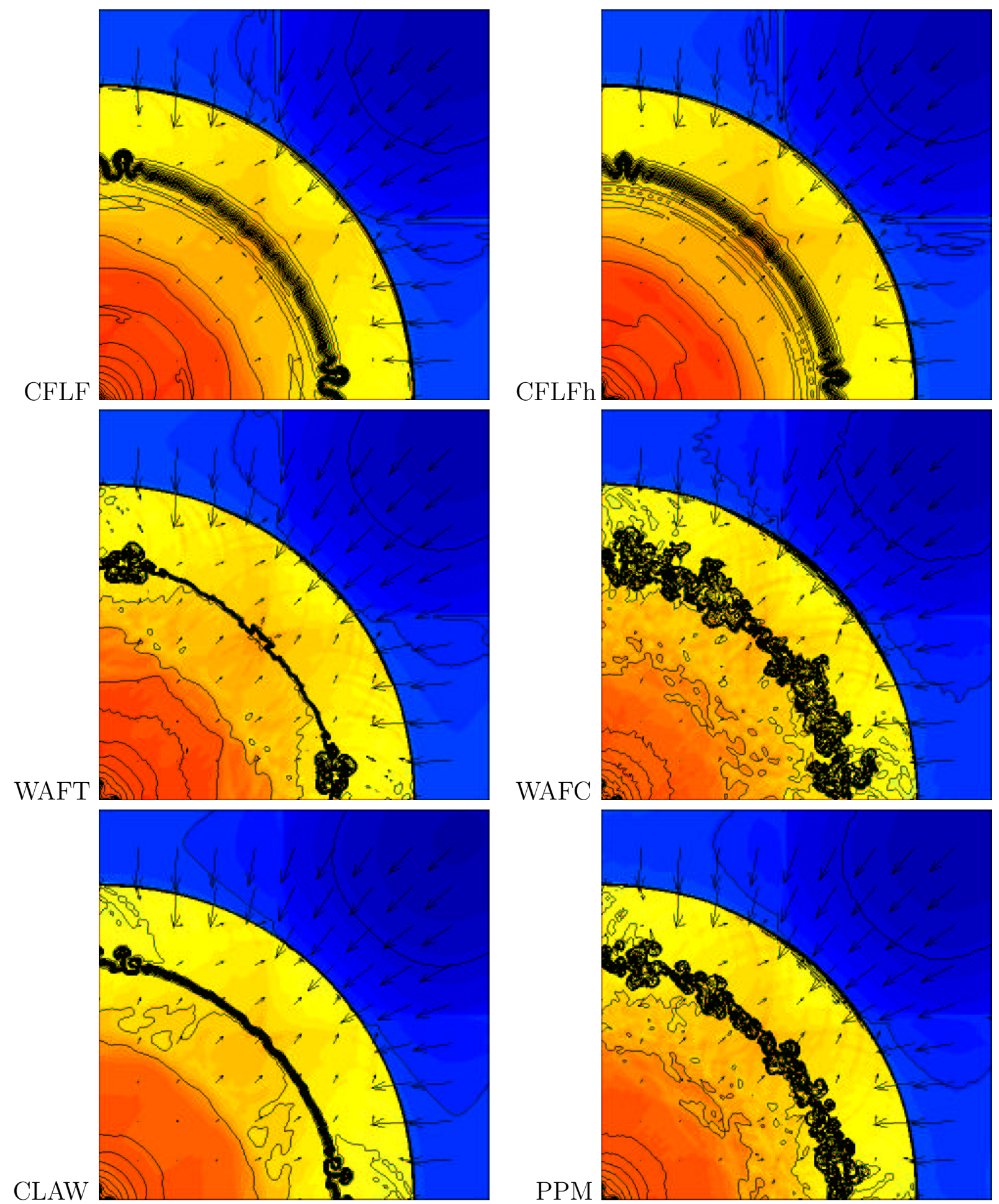

Figure 34: Results for the explosion problem by six schemes. Color pressure map is overlayed by 27 density contours (0.08 to 0.21 step 0.005$)$ and velocity arrows. Computations were done on the square $(x, y) \in(0,1.5) \times(0,1.5)$ with $400 \times 400$ grid till time $T=3.2$. 


\section{Explosion}

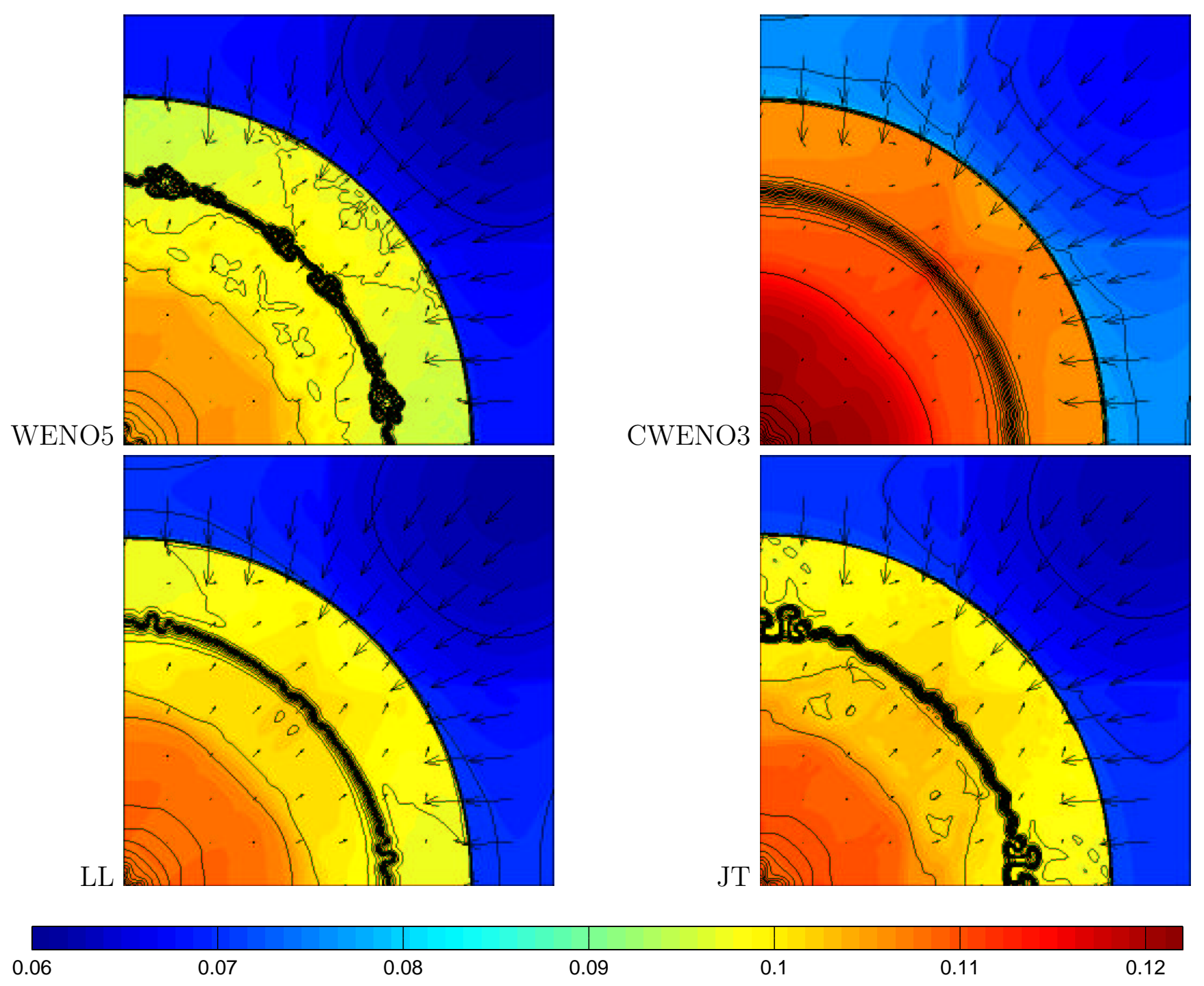

Figure 35: Results for the explosion problem by remaining four schemes with pressure colorbar. Color pressure map is overlayed by 27 density contours (0.08 to 0.21 step 0.005$)$ and velocity arrows. Computations were done on the square $(x, y) \in(0,1.5) \times(0,1.5)$ with $400 \times 400$ grid till time $T=3.2$. 


\section{Final remarks}

We have taken a collection of schemes that are representative of most of the basic approaches to approximating the Euler equations by finite difference methods - including the use of central differencing, eigenvector decomposition, dimensional splitting, Runge-Kutta time stepping, limiting, hybridization, and Riemann solvers. We have applied these to a suite of problems in one and two dimensions. It is clear that some methods appear to work better than others on a specific problem, but no one scheme has shown itself to be superior on all of them, which should come as no surprise. Some schemes are much faster than others, but not too much should be read into this since in many cases we had to manipulate the data to fit into our framework.

We present this to the computational fluid dynamics community with the hope that it will contribute to the still vigorous research being done there.

\section{Acknowledgements}

Many people have helped along the way in this project. Particular thanks are owing to Paul Woodward who presented a careful appraisal of some of the problems and of the behavior of PPM. Eleuterio Toro generously shared with us the WAF code from the Numerica library. We also would like to acknowledge Tariq Aslam, Guan-Shan Jiang, Milan Kuchařík, Alexander Kurganov, Randall LeVeque, Xu-Dong Liu, Len Margolin, James Quirk, Misha Shashkov, Chi-Wang Shu, and Eitan Tadmor. This research was supported by the U. S. Department of Energy under contract W-7405-ENG-36. R. Liska was supported in part by the Czech Grant Agency grant 201/00/0586 and would like to thank the Institute for Geophysics and Planetary Physics (IGPP) for hosting his visits at Los Alamos National Laboratory.

\section{References}

[1] J. Smoller. Shock Waves and Reaction Diffusion Equations. Springer-Verlag, New York, 1994. Grundlehren der mathematischen Wissenschaften 258 (Second Edition).

[2] C. M. Dafermos. Hyperbolic Conservation Laws in Continuum Physics. Springer-Verlag, Berlin, 1991. Grundlehren der mathematischen Wissenschaften 325.

[3] E. Godlewski and P-A. Raviart. Numerical Approximation of Hyperbolic Systems of Conservation Laws. Springer-Verlag, 1996. Applied Mathematical Sciences 118.

[4] Harald Hanche-Olsen, Helge Holden, and Knut-Andreas Lie. Conservation laws preprint serve. http://www.math.ntnu.no/conservation.

[5] D. Kröner and M. Ohlberger. A posteriori error estimates for upwind finite volume schemes for nonlinear conservation laws in multi dimensions. Math. Comp., 69:25-39, 1999.

[6] P. J. Roache. Computational Fluid Dynamics. Hermosa Press, 1982.

[7] R.J. LeVeque. Numerical Methods for Conservation Laws. Birkhauser Verlag, Basel, 1990.

[8] C.B. Laney. Computational Gasdynamics. Cambridge University Press, Cambridge, UK, 1998. 
[9] G. A. Sod. A survey of several finite difference schemes for hyperbolic conservation laws. J. Comp. Phys., 27:1-31, 1978.

[10] E.F. Toro. Riemann Solvers and Numerical Methods for Fluid Dynamics. Springer-Verlag, Berlin, Heidelberg, 1997.

[11] G. Strang. On the construction and comparison of difference schemes. SIAM J. Numer. Anal., 5(3):506-517, 1968.

[12] R. Liska and B. Wendroff. Composite schemes for conservation laws. SIAM J. Numer. Anal., 35(6):2250-2271, 1998.

[13] R. Liska and B. Wendroff. Analysis and computation with stratified fluid models. J. Comp. Phys., 137:212-244, 1997.

[14] R. Liska and B. Wendroff. Composite centered schemes for multidimensional conservation laws. In M. Fey and R. Jeltsch, editors, Hyperbolic Problems: Theory, Numerics, Applications, Seventh International Conference in Zürich, February 1998, Vol. II, pages 661-670, Basel, 1999. Birkhäuser, ISNM Vol.130.

[15] R. Liska and B. Wendroff. Two-dimensional shallow water equations by composite schemes. Int. J. Numer. Meth. Fluids, 30:461-479, 1999.

[16] R. Liska and B. Wendroff. Shallow water conservation laws on a sphere. In Eighth International Conference on Hyperbolic Problems Theory, Numerics, Applications (HYP2000), Magdeburg, 2000. (accepted).

[17] M. Shashkov and B. Wendroff. A composite scheme for gas dynamics in lagrangian coordinates. J. Comp. Phys., 150:502-517, 1999.

[18] K. Kozel, M. Janda, and R. Liska. Composite schemes on triangular meshes. In Eighth International Conference on Hyperbolic Problems Theory, Numerics, Applications (HYP2000), Magdeburg, 2000. (accepted).

[19] A. Harten. The artificial compression method for computation of shocks and contact discontinuities: Iii self adjusting hybrid schemes. Mathematics of Computation, 32:363-389, 1978.

[20] G.S. Jiang and E. Tadmor. Non-oscillatory central schemes for multidimensional hyperbolic conservation laws. SIAM Journal on Scientific Computing, 19:1892-1917, 1998.

[21] H. Nessyahu and E. Tadmor. Non-oscillatory central differencing for hyperbolic conservation laws. J. Comp. Phys., 87(2):408-463, 1990.

[22] X.D. Liu and P.D. Lax. Positive schemes for solving multi-dimensional hyperbolic systems of conservation laws. CFD Journal, 5(1):1-24, 1996. http://wwW.math.ucsb.edu/〜xliu/publication/paper/positive1.pdf.

[23] P. D. Lax and X.-D. Liu. Solution of two dimensional Riemann problem of gas dynamics by positive schemes. SIAM J. on Scientific Comp., 19(2):319-340, 1998. 
[24] R.J. LeVeque. Wave propagation algorithms for multi-dimensional hyperbolic systems. J. Comp. Phys., 131:327-353, 1997.

[25] R.J. LeVeque. High-resolution conservative algorithms for advection in incompressible flow. SIAM J. Numer. Anal., 33:627-665, 1996.

[26] R.J. LeVeque. Clawpack version 4.0 user's guide. Technical report, University of Washington, Seatle, 1999. http://www.amath.washington.edu/〜 claw/.

[27] J.J. Quirk. personal communication. 2001.

[28] J.J. Quirk. An Adaptive Grid Algorithm for Computational Shock Hydrodynamics. PhD thesis, College of Aeronautics, Cranfield Institute of Technology, 1991. http://www.galcit.caltech.edu/〜jjq/doc/amr_sol/thesis.

[29] E.F. Toro. A weighted average flux method for hyperbolic conservation laws. Proc. R. Soc. London A, 423:401, 1989.

[30] S.J. Billett and E.F. Toro. On waf-type schemes for multidimensional hyperbolic conservation laws. J. Comp. Phys., 130:1-24, 1997.

[31] E.F. Toro. Numerica a library of source codes for teaching, research and applcations:, 2000. http://www.numeritek.com/numerica_software.html.

[32] B. Einfeldt. On godunov-type methods for gas dynamics. SIAM J. Numer Anal., 25:294-318, 1988.

[33] Guan-Shan Jiang and Chi-Wang Shu. Efficient implementation of weighted eno schemes. J. Comp. Phys., 126:202-228, 1996.

[34] A. Harten and S. Osher. Uniformly high-order accurate nonoscilatory schemes. SIAM J. Numer. Anal., 24:279-309, 1987.

[35] P. Woodward and P. Colella. The numerical simulation of two-dimensional fluid flow with strong shocks. J. Comp. Phys., 54:115-173, 1984.

[36] P.R. Woodward, B. Edgar, and S.E. Anderson. An introduction to the piecewise parabolic method (ppm) library. Technical report, University of Minnesota, Minneapolis.

[37] Ppmlib library. University of Minnesota. http://www.lcse.umn.edu/PPMlib/.

[38] W. F. Noh. Errors for calculations of strong shocks using an artificial viscosity and artificial heat flux. J. Comp. Phys., 72:78-120, 1987.

[39] Milan Kuchařík. personal communication. 2001.

[40] P. Woodward. personal communication. 2001.

[41] R. Saurel and R. Abgrall. A simple method for compressible multifluid flows. SIAM J. Sci. Comput., 21:1115-1145, 1999.

[42] X.D. Liu. personal communication. 2001. 
[43] C. W. Schulz-Rinne, J. P. Collins, and H. M. Glaz. Numerical solution of the Riemann problem for two-dimensional gas dynamics. SIAM J. Sci. Comput., 14:1394-1414, 1993.

[44] A. Kurganov and E. Tadmor. Solution of two-dimensional riemann problems for gas dynamics without riemann problem solvers. CAM 00-34, UCLA, Los Angeles, 2000.

[45] William J. Rider. Revisiting wall heating. J. Comp. Phys., 162:395 - 410, 2000.

[46] J. Grove and V. Mousseau. personal communication. 2000.

[47] T. Aslam. personal communication. 2001.

[48] W.H. Hui, P.Y. Li, and Z.W. Li. A unified coordinate system for solving the two-dimensional euler equations. J. Comp. Phys., 153:596-637, 1999.

[49] S.C. Chang, X.Y. Wang, and C.Y. Chow. The space-time conservation element and solution element method: A new high resolution and genuinely multidimensional paradigm for solving conservation laws. J. Comp. Phys., 160:89-136, 1999. 